Old Dominion University

ODU Digital Commons

Mathematics \& Statistics Theses \&

Dissertations

Mathematics \& Statistics

Summer 1987

\title{
Large Deviation Local Limit Theorems for Ratio Statistics
}

Sanjeev V. Sabnis

Old Dominion University

Follow this and additional works at: https://digitalcommons.odu.edu/mathstat_etds

Part of the Applied Statistics Commons, and the Probability Commons

\section{Recommended Citation}

Sabnis, Sanjeev V.. "Large Deviation Local Limit Theorems for Ratio Statistics" (1987). Doctor of Philosophy (PhD), Dissertation, Mathematics \& Statistics, Old Dominion University, DOI: 10.25777/07wjb034

https://digitalcommons.odu.edu/mathstat_etds/104

This Dissertation is brought to you for free and open access by the Mathematics \& Statistics at ODU Digital Commons. It has been accepted for inclusion in Mathematics \& Statistics Theses \& Dissertations by an authorized administrator of ODU Digital Commons. For more information, please contact digitalcommons@odu.edu. 


\title{
LARGE DEVIATION LOCAL LIMIT THEOREMS \\ FOR RATIO STATISTICS
}

\author{
by \\ Sanjeev V. Sabnis \\ B.Sc. June 1979, University of Poona, India \\ M.Sc. June 1981, University of Poona, India \\ A dissertation Submitted to the Faculty of \\ Old Dominion University in Partial Fulfillment of the \\ Requirements for the Degree of \\ DOCTOR OF PHILOSUPHY \\ COMPUTATIONAL AND APPLLIED MATHEMATICS
}

\section{OLD DOMINION UNIVERSITY}

August, 1987

Approved by:

$\overline{\text { DIn N. R. Chragahtv (Difector) }}$

Dr. Ram C. Dahiva

Dr. John P. Morgan

Dr. Edward P. Markowski 


\section{Abstract \\ LARGE DEVIATION LOCAL LIMIT THEOREMS FOR RATIO STATISTICS \\ Sanjeev V. Sabnis \\ Old Dominion University, 1987 \\ Director: Dr. N. R. Chaganty}

Let $\left\{T_{n}, n \geq 1\right\}$ be an arbitrary sequence of non-lattice random variables and $\left\{S_{n}, n \geq 1\right\}$ be another sequence of positive non-lattice random variables. Let the two sequences be independent. Let $\phi_{1 n}$ and $\phi_{2 n}$ be the moment genereating functions of $\left\{T_{n}, n \geq 1\right\}$ and $\left\{S_{n}, n \geq 1\right\}$ respectively. Let $\left\{a_{n}\right\}$ be a sequence of real numbers such that $a_{n} \rightarrow \infty$. Let

$$
\psi_{1 n}(z)=\frac{1}{a_{n}} \log \phi_{1 n}(z)
$$

and

$$
\psi_{2 n}(z)=\frac{1}{a_{n}} \log \phi_{2 n}(z)
$$

Under some mild and easily verifiable conditions on $\phi_{1 n}$ and $\phi_{2 n}$, which imply that $\left(T_{n}-E\left(T_{n}\right)\right) / a_{n} \rightarrow 0$ in probability and $\left(S_{n}-E\left(S_{n}\right)\right) / a_{n} \rightarrow$ $c$ in probability for some $c>0$, we obtain a large deviation local limit theorem for the ratio statistic $R_{n}=T_{n} / S_{n}$, that is, we obtain an asymptotic expression for the density, $f_{n}\left(r_{n}\right)$, of the ratio statistic $R_{n}=T_{n} / S_{n}$ at the point $r_{n}$ where $\left\{r_{n}, n \geq 1\right\}$ is a bounded sequence of real numbers. This expression is given by

$$
\begin{aligned}
f_{n}\left(r_{n}\right)= & \frac{a_{n} \psi_{2 n}^{\prime}\left(-r_{n} \tau_{n}\right)}{\left[2 \pi a_{n}\left[\psi_{1 n}^{\prime \prime}\left(\tau_{n}\right)+r_{n}^{2} \psi_{2 n}^{\prime \prime}\left(-r_{n} \tau_{n}\right)\right]\right]^{1 / 2}} \\
& \times \exp \left(a_{n}\left[\psi_{1 n}\left(\tau_{n}\right)+\psi_{2 n}\left(-r_{n} \tau_{n}\right)\right]\right)\left[1+O\left(1 / a_{n}\right)\right]
\end{aligned}
$$


where $\tau_{n}$ is such that

$$
\psi_{1 n}^{\prime}\left(\tau_{n}\right)-r_{n} \psi_{2 n}^{\prime}\left(-r_{n} \tau_{n}\right)=0
$$

When $S_{n}$ is degenerate at $n$, our result reduces to the result of ChagantySethuraman (Ann. of Probab., (1985), vol. 13, 97-113). We obtain similar asymptotic expressions for the probability density functions (p.d.f.) or probability mass functions (p.m.f.) of ratio statistics arising from different combinations of non-lattice and lattice random variables $T_{n}$ and $S_{n}$.

We also present the corresponding results for the ratio of sums of i.i.d. random variables along with some interesting applications. In all of these applications, the p.d.f.'s (or p.m.f.'s) do not have closed form expressions. However, our results provide simple computable approximations involving moment generating functions for such p.d.f.'s (or p.m.f.'s). We verify our result for the $F$ statistic, whose exact density is available. Our approximation coincides with the exact expression except for the normalizing constant. However, we show that our approximation is asymptotically equivalent to the exact density.

Let $\left\{T_{n}, n \geq 1\right\}$ be an arbitrary sequence of random vectors and $\left\{S_{n}>0, n \geq 1\right\}$ be an arbitrary sequence of random variables. In Chapter III, we obtain an asymptotic expression for the density of the vector $R_{n}=T_{n} / S_{n}$. A result which obtains an asymptotic expression for the tail probability, $P\left(R_{n}>r_{n}\right)$, is known as a strong large deviation result. In Chapter $I V$, we obtain strong large deviation results for statistics which can be expressed as the ratio of two independent sequences of non-lattice as well as lattice random variables. 
my parents 


\section{Acknowledgments}

I would like to thank Dr. N. R. Chaganty for introducing me to the area of large deviation limit theory and for providing much of his own time during this work. This dissertation is a result of his inspiring guidance. I am thankful to Dr. Ram Dahiya, Dr. John Morgan, Dr. Edward Markowski for being on my thesis committee. I would also like to extend my thanks to Dr. John Tweed for making the departmental computer facilities available to me.

Thanks are also due to Dr. R. V. Rammamorthy of Michigan State University who recommended me to Old Dominion University.

Finally, I am grateful to my wife, Sheela whose patience and constant encouragement helped me to complete this seemingly unending project. 


\section{Contents}

1 Introduction and Summary 1-1

2 Large Deviation Local Limit Theorems 2-8

2.1 Introduction . . . . . . . . . . . . . . 2-8

2.2 Local limit theorems for arbitrary sequence of random variables .......................... 2-12

2.3 Large deviation local limit theorems for ratio statistics . . . 2-28

2.4 Large deviation local limit theorems for ratio statistics of lattice random variables . . . . . . . . . . . 2-51

2.5 Applications . . . . . . . . . . . . . 2-57

3 Large deviation local limit theorems for random vectors 3-64

3.1 Definitions and Preliminaries. . . . . . . . . . . 3-64

3.2 Local limit theorems for random vectors . . . . . . . . 3-66

3.3 Large deviation local limit theorems for lattice random vec-

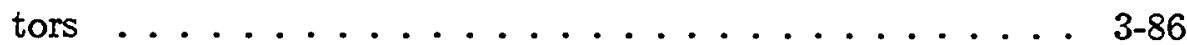

4 Strong large deviation results for ratio statistics 4-92

vi 
4.1 Introduction . . . . . . . . . . . . . . 4- 4- .

4.2 Non-lattice case . . . . . . . . . . . . . 4-93

4.3 The Lattice case . . . . . . . . . . . . . 4-97

$\begin{array}{ll}\text { References } & 100\end{array}$ 


\section{Chapter 1}

\section{Introduction and Summary}

The origin of probability theory dates back to the middle of the 17th century. Since then limit theorems have become the core of probability theory as they are quite useful in understanding the random phenomena present in all our sciences. The history of the limit theorems begins with the limit theorems of Bernoulli (1713). Then followed the limit theorems of de Moivre and Laplace. Later on, Poisson succeeded in obtaining generalizations of the theorems of Bernoulli, de Moivre and Laplace. In today's context these theorems are referred to as the laws of large numbers, one of the fundamental propositions of the theory of probability. We state these results below in Theorems 1.1 and 1.2 which are now known as Weak Law of Large Numbers (WLLN) and Strong Law of Large Numbers (SLLN) respectively. The WLLN refers to convergence in probability whereas the SLLN refers to almost sure convergence of averages of random variables.

1-1 
Theorem 1.1 (WLLN). Let $\left\{X_{n}, n \geq 1\right\}$ be pairwise independent and identically distributed random variables with finite mean $E\left(X_{1}\right)$. Then we have

$$
\frac{S_{n}}{n} \rightarrow E\left(X_{1}\right)
$$

in probability.

Theorem 1.2 (SLLN). Let $\left\{X_{n}, n \geq 1\right\}$ be a sequence of i.i.d. random variables with finite mean $E\left(X_{1}\right)$. Then we have

$$
\frac{S_{n}}{n} \rightarrow E\left(X_{1}\right)
$$

almost surely.

Theorems 1.1 and 1.2 are due to Khintchine and Kolmogorov respectively.

Chebyshev, a Russian Mathematician, made major contributions to the theory of probability by introducing new techniques to prove the theorems concerning sums of arbitrarily distributed independent random variables. For example, under the assumption of existence of moments of all orders (by utilizing the methods of moments), Chebyshev gave different versions of the proof of the Central Limit Theorem (CLT) (see Theorem 1.3 below for a version of the CLT) for sums of independent but arbitrarily distributed random variables. The CLT deals with convergence in distribution of normalized sums of random variables. Then around 1900, Lyapunov proved CLT with substantially weaker restrictions than those required by Chebyshev. He proved the theorem utilizing the method of characteristic func- 
tions, developed by himself, rather than the method of moments used by Chebyshev. We give the simplest form of the CLT in Theorem 1.3.

Theorem 1.3 (CLT). Let $\left\{X_{n}, n \geq 1\right\}$ be a sequence of i.i.d. random variables with $E\left(X_{1}\right)=\mu$ and $\operatorname{var}\left(X_{1}\right)=\sigma^{2}$ and distribution function $F$. Let $F_{n}$ denote the distribution function of the normalized sum, that is, the distribution function of $\left(S_{n}-n \mu\right) / \sqrt{n} \sigma$, where $S_{n}=X_{1}+\cdots+X_{n}$. Then

$$
F_{n}(x) \rightarrow \Phi(x)
$$

uniformly in $x$ where $\Phi(x)$ denotes the distribution function of the standard normal random variable.

While the CLT gives the limit of a sequence of distribution functions $F_{n}$, it is quite inadequate in practice. In many applications we are interested not only in the convergence of $F_{n}(x)$ but also in the error of approximating $F_{n}(x)$ by $\Phi(x)$. This question was addressed by several authors including Cramer (1938), Berry(1941), Esseen(1943, 1945) and Chernoff (1952). Berry and Essen established an upperbound for the error $e_{n}(x)=\left|F_{n}(x)-\Phi(x)\right|$, which is uniform in $x$. While the upperbound for the error $e_{n}(x)$ is quite sharp for moderate values of $x$, however, for large values of $x$, both $F_{n}(x)$ and $\Phi(x)$ are close to 1 and the upperbound overestimates the error $e_{n}(x)$. Chernoff(1952) initiated the study of the tail of $F_{n}(x)$. He showed that if $X_{1}$ has a finite moment generating function in a neighbourhood of zero, then the probability that $S_{n} / n$ deviates from $E\left(X_{1}\right)$ by a small amount $\varepsilon>0$ tends to zero exponentially fast as $n \rightarrow \infty$. The 
event $E_{n}=\left\{\left|S_{n} / n-E\left(X_{1}\right)\right|>\epsilon\right\}$ is known as a large deviation event since it represents the deviation of $S_{n} / n$ away from the mean. The probability of occurrence of $E_{n}$ is known as a large deviation probability. Therefore, Chernoff's theorem is known as a large deviation limit theorem. In recent years, limit theorems for large deviations have become a vital part of the theory of probability. The theory of large deviations forms a natural complement to the law of large numbers stated in Theorems 1.1 and 1.2.

Among the different kinds of limit theorems, the local limit theorems are also of great importance. These theorems deal with the convergence of a sequence of density functions to another density function as well as with asymptotic expansions for a sequence of density functions. One should note that the hypothesis of the CLT does not guarantee the convergence of the corresponding density functions to the standard normal density. In fact, such a convergence takes place almost everywhere only when $F_{n}$ is nonsingular for some $n$.

Let $\left\{X_{n}, n \geq 1\right\}$ be a sequence of independent and identically distributed random variables. Let $S_{n}=X_{1}+\cdots+X_{n}$ be the $n^{\text {th }}$ partial sum. Let $f_{n}$ denote the density function of $S_{n} / \sqrt{n}$. Richter proved an important local limit theorem for large deviations for sums of i.i.d. random variables, that is, an asymptotic expansion for $f_{n}\left(x_{n}\right)$ when $x_{n}$ is allowed to increase with $n$. Chaganty and Sethuraman (1985) generalized Richter's theorem to an arbitrary sequence of random variables thereby increasing the applicability of Richter's theorem to new classes of statistics. We state the result of Chaganty and Sethuraman in Theorem 2.2.3 of Chapter II. 
The goal of this dissertation is to generalize Theorem 2.2.3 to the ratio statistic $R_{n}=T_{n} / S_{n}$, where $\left\{S_{n}, n \geq 1\right\}$ is an arbitrary sequence of positive random variables independent of $\left\{T_{n}, n \geq 1\right\}$. In the special case where $T_{n}$ and $S_{n}$ are partial sums of independent random variables our result simplifies to the heuristic conclusion of Daniels (1954).

The problem of obtaining the limit for $\log P\left(T_{n} \geq x_{n}\right)$, where $x_{n} \rightarrow \infty$ at a suitable rate as $n \rightarrow \infty$, has been studied by several authors including Cramer (1938), Chernoff (1952), and Ellis (1984). Chaganty and Sethuraman chose to call these results of Cramír, Chernoff and Ellis as weak large deviation results since they give an asymptotic expansion for $\log P\left(T_{n} \geq x_{n}\right)$ rather than an approximation for $P\left(T_{n} \geq x_{n}\right)$. An approximation for $P\left(T_{n} \geq x_{n}\right)$ is known as a strong large deviation result. One of the earliest strong large deviation results when $T_{n}$ is the sum of i.i.d. random variables was obtained by Bahadur and Ranga Rao (1960). Recently, Chaganty and Sethuraman (1986) extended the result of Bahadur and Ranga Rao to an arbitrary sequence of random variables $\left\{T_{n}, n \geq 1\right\}$. In this dissertation we present analogous results for statistics which can be expressed as the ratio of two independent sequences of non-lattice as well as the ratio of two independent sequences of lattice random variables.

This dissertation has been organised as follows:

Chapter II consists of five sections. In Section 2.1 we present the background material. In Section 2.2 we state Chaganty-Sethuraman's results for an arbitrary sequence of non-lattice random variables. We also state and outline the proof of Chaganty-Sethuraman's theorem with Condition (iii') 
in Theorem 2.2.3 replaced by another simple and easily verifiable Condition (iii"). As an application we obtain a large deviation local limit theorem for the Wilcoxon signed rank statistic under the null hypothesis. Section 2.3 contains the main results of this dissertation. Let $\left\{T_{n}, n \geq 1\right\}$ be an arbitrary sequence of non-lattice random variables and $\left\{S_{n}, n \geq 1\right\}$ be another arbitrary sequence of positive non-lattice random variables. Under some mild conditions on $\phi_{1 n}$ and $\phi_{2 n}$, the moment generating functions of $T_{n}$ and $S_{n}$ respectively, we obtain in Theorem 2.3.1 an asymptotic expression for the density of the ratio statistic $R_{n}=T_{n} / S_{n}$. We also obtain an asymptotic expression in Theorem 2.3.12 for the density of the ratio statistic in the case where $T_{n}$ is non-lattice and $S_{n}$ is lattice. In Section 2.4 we obtain similar results in Theorems 2.4.1 and 2.4.2 when $T_{n}$ and $S_{n}$ both are lattice random variables and when $T_{n}$ is lattice and $S_{n}$ is non-lattice respectively. In the case where $T_{n}$ and $S_{n}$ are sums of i.i.d. random variables, the conditions of the Theorems 2.3.1, 2.3.12, 2.4.1 and 2.4.2 are very much simplified. We present the corresponding results for the ratios of sums of i.i.d. random variables in Theorems $2.3 .13,2.3 .14,2.4 .3$ and 2.4 .4 respectively. In Section 2.5 we provide three examples illustrating different occurrences of non-lattice and lattice $T_{n}$ and $S_{n}$. For simplicity, in these examples we let $T_{n}$ and $S_{n}$ to be sums of i.i.d. random variables. We also study our approximation for the $\mathrm{F}$ statistic, whose exact density is available. Our approximation coincides with the exact expression except for the normalizing constant. However, our approximation is asymptotically equivalent to the exact density. 
Chapter III contains large deviation local limit theorems for an arbitrary sequence of random vectors. Let $\left\{T_{n}, n \geq 1\right\}$ be an arbitrary sequence of random vectors and $\left\{S_{n}, n \geq 1\right\}$ be an arbitrary sequence of positive random variables. An asymptotic expression for the density of the ratio statistic, $R_{n}=T_{n} / S_{n}$ is given.

In Chapter IV, we present strong large deviation results for statistics which can be expressed as the ratio of two independent sequences of nonlattice as well as the ratio of independent sequences of lattice random variables. 


\section{Chapter 2}

\section{Large Deviation Local Limit}

\section{Theorems}

\subsection{Introduction}

The very basic probability limit theorems are the weak law of large numbers (WLLN), the strong law of large numbers (SLLN) and the Central limit theorem (CLT). The WLLN refers to convergence in probability where as the SLLN refers to almost sure convergence of the averages of random variables. The CLT deals with convergence in distribution of normalized sums of random variables. If $\left\{X_{n}, n \geq 1\right\}$ is a sequence of i.i.d. random variables with $E\left(X_{1}\right)=\mu, \operatorname{Var}\left(X_{1}\right)=\sigma^{2}$ and distribution function $F$ and further if $F_{n}$ denotes the distribution function of the normalized sum $\left(S_{n}-n \mu\right) / \sqrt{n} \sigma$ then the CLT asserts that,

$$
F_{n}(x) \rightarrow \Phi(x)
$$


uniformly in $x$, where $\Phi(x)$ denotes the distribution Iunction of the standard normal random variable.

The CLT raises a number of questions. For instance,

1) Do the partial sums of random variables always converge in distribution to the normal distribution?

2) How much error is committed in the approximation of $F_{n}(x)$ by $\Phi(x)$ ?

3) Does the hypothesis of the CLT imply convergence of the corresponding density functions to the normal density?

The answer to the first question is negative. It leads to the theory of infinite divisibility. As regards to the second question different authors have obtained asymptotic expansions, as well as bounds for the error $e_{n}(x)=\left|F_{n}(x)-\Phi(x)\right|$ for fixed $x$. Theorem 2.1 .1 below gives an asymptotic expansion for $e_{n}(x)$ and Theorem 2.1.2 gives a bound on the error term $e_{n}(x)$. Theorems 2.1.1 and 2.1.2 are due to Esseen(1945), Berry (1941) and Esseen (1943) respectively.

Theorem 2.1.1 (Esseen). If $F$ is non-lattice and has a finite third moment

$$
\mu_{3}=\int_{-\infty}^{\infty} x^{3} d F(x)
$$

then

$$
e_{n}(x)=\frac{\mu_{3}\left(1-x^{2}\right)}{6 \sigma^{3} \sqrt{2 \pi n}} \exp \left(-x^{2} / 2\right)+o(1 / n)
$$

uniformly in $x$. 
Theorem 2.1.2 (Berry and Esseen). Let $E\left(X_{1}\right)=0$ and let $\rho=E\left|X_{1}\right|^{3}$ be finite. Then for all $x$ and $n$

$$
\left|e_{n}(x)\right| \leq \frac{33}{4} \frac{\rho}{\sigma^{3} \sqrt{n}}
$$

The constant 33/4 was first replaced by 0.91 in Zolotarev (1967) and subsequently by 0.7975 in Van Beeck (1972). For fixed $n$ and for large values of $x$, the quantities $F_{n}(x)$ and $\Phi(x)$ both become close to 1 and the bound given in Theorem 2.1.2 becomes impracticable. The problem of studying the error of approximation was approached differentiy by Cramer(1938). He obtained an estimate of the ratio of the tail probabilities,

$$
\frac{\left[1-F_{n}\left(x_{n}\right)\right]}{\left[1-\Phi\left(x_{n}\right)\right]}
$$

for $x_{n}=O(\sqrt{n})$, assuming that the moment generating function of $X_{1}$ exists. Petrov (1954) extended this result to the case of non identically distributed random variables.

A counter example given in the book of Gnedenko and Kolmogorov (1954), page 223 answers the third question in the negative. A close examination of the example reveals that the convergence fails to take place only in a neighborhood of the origin. However, a result due to Ranga Rao and Varadarajan (1960) guarantees convergence almost everywhere. We state this result in Theorem 2.1.3 below.

Theorem 2.1.3. A necessary and sufficient condition for $f_{n}(x)$ to converge to $\phi(x)$ almost everywhere is that $F_{n}$ is nonsingular for some $n$. 
Theorem 2.1.3 is known as a local limit theorem for densities.

The problem of obtaining an estimate of the ratio in (2.3) of the tail probabilities can be characterized as the problem of large deviation. If $\left\{T_{n}, n \geq 1\right\}$ is a sequence of random variables, an approximation to $P\left(T_{n} \geq x_{n}\right)$ where $x_{n} \rightarrow \infty$, is known as a Strong large deviation result whereas an approximation to $k_{n}\left(x_{n}\right)$, the probability density function of $T_{n}$ at $x_{n}$, is known in the literature as the large deviation local limit theorem. The result of the latter kind was first obtained by Richter (1957) for the sums of i.i.d. random variables. This result was stated in Richter's paper in terms of Cramer series. Recently, Chaganty-Sethuraman showed that the same result can be rewritten in terms of the so called large deviation rate. Large deviation local limit theorems for sums of independent, non-identically distributed lattice and non-lattice random variables were also obtained by Moskvin (1972) and by MacDonald (1979). Chaganty-Sethuraman (1985) extended Richter's result for an arbitrary sequence of random variables, for both non-lattice and lattice cases. They have demonstrated the applicability of the large deviation local limit theorems for statistics that occur in nonparametric inference and also in establishing limit theorems for some probabilistic models occuring in statistical mechanics(see Chaganty and Sethuraman (1987)).

In this chapter we first state the local limit Theorems 2.2.1 and 2.2.3 due to Richter (1957) and Chaganty-Sethuraman (1985), respectively. As noted by Chaganty-Sethuraman (1985) Condition (iii') of Theorem 2.2.3 was difficult to verify in examples. In Theorem 2.2.8 we establish Theorem 
2.2.3, replacing Condition (iii') by another easily verifiable Condition (iii"). The main goal of this chapter is to present similar large deviation local limit theorems for statistics which can be expressed as the ratio of two independent random variables. These results are obtained in Sections 2.3 and 2.4. We treat the case of the ratio of two non-lattice arbitrary random variables in Theorem 2.3.1. Theorems 2.3.12, 2.4.1 and 2.4.2 cover the cases when one of the random variables is non-lattice and the other one is lattice. The conditions of our theorems are much simplified in the case of ratios of sums of i.i.d. random variables. We state these results in Theorems 2.3.13, 2.3.14, 2.4.3 and 2.4.4. In Section 2.5 we provide a number of applications of our theorems.

\subsection{Local limit theorems for arbitrary se- quence of random variables}

Richter (1957) proved a large deviation local limit theorem involving the Cramer series for the sequence $\left\{X_{n}, n \geq 1\right\}$ of i.i.d. random variables. Chaganty-Sethuraman (1985) restated Richter's result in terms of the large deviation rate and extended the result to an arbitrary sequence of random variables not necessarily sums of i.i.d. random variables. We present below statements of these theorems.

Theorem 2.2.1 (Richter). Let $\left\{X_{n}, n \geq 1\right\}$ be a sequence of i.i.d. random variables having common distribution function $F$ with $E\left(X_{1}\right)=0$ and 
$\operatorname{Var}\left(X_{1}\right)=\sigma^{2}$. Let $S_{n}=X_{1}+X_{2}+\cdots+X_{n}$. Let $f_{n}$ be the p.d.f. of $S_{n} / \sqrt{n}$. Assume the following conditions:

(1) There exists a positive number $A$ such that

$$
\int_{-\infty}^{\infty} \exp (s x) d F(x)<\infty
$$

for all s with $|s|<A$.

(2) There exists an $n_{0}$ such that the distribution function of $S_{n}=X_{1}+$ $X_{2}+\cdots+X_{n}$ is absolutely continuous with a bounded derivative for $n \geq n_{0}$. Then, if $x>1$ and $x=O(\sqrt{n})$, we have, as $n \rightarrow \infty$

$$
f_{n}(x)=\phi(x) \exp \left(\frac{x^{3}}{\sqrt{n}}\right) \lambda\left(\frac{x}{\sqrt{n}}\right)\left[1+O\left(\frac{x}{\sqrt{n}}\right)\right]
$$

where $\lambda(t)$ is a power series converging for all sufficiently small values of $|t|$ and $\phi(x)$ is the density of the standard normal random variable.

The next Theorem 2.2.2 is essentially a reformulation of Theorem 2.2.1 in terms of the large deviation rate function $\gamma$ defined below in Theorem 2.2.2. One should also note the conditions of Theorem 2.2.1 are weaker than those of Theorem 2.2.2. Here and throughout this dissertation $\log z$ denotes the principal logarithm of the complex number $z$.

Theorem 2.2.2. Let $\left\{X_{n}, n \geq 1\right\}$ be an i.i.d. sequence of random variables with common distribution function $F$ and analytic characteristic function $\phi(z), z \in C$, where $C$ is the set of all complex numbers. Let $\psi(z)=\log \phi(z)$. 
Let $I$ denote the interval $(-a, a)$, for fixed positive number $a$. Let $m$ be $a$ real number and $a_{1}$ be such that $0<a_{1}<a$. Let $\tau$ be such that $\psi^{\prime}(\tau)=m$ and denote $\psi^{\prime \prime}(\tau)=\sigma^{2}>0$. Let $m_{n} \rightarrow m$. Assume the following conditions:

(i) There exists $\beta>0$ such that $|\psi(s)|<\beta$, for all $s \in I$.

(ii) There exists $\tau_{n} \in\left(-a_{1}, a_{1}\right)$ suci, that $\psi^{\prime}\left(\tau_{n}\right)=m_{n}$ for $n \geq 1$.

(iii) There exists $A<\infty$ such that

$$
\sup _{s \in I} \int_{-\infty}^{\infty}\left|\frac{\phi(s+i t)}{\phi(s)}\right| d t \leq A .
$$

Define $\gamma(x)=\sup _{s \in I}[x s-\psi(s)]$. Let $v_{n}$ denote the probability density function of $S_{n} / n$, where $S_{n}=X_{1}+X_{2}+\cdots+X_{n}$. Then,

$$
v_{n}\left(m_{n}\right)=\frac{\sqrt{n}}{\sqrt{2 \pi} \sigma} \exp \left(-n \gamma\left(m_{n}\right)\right)[1+O(1 / n)]
$$

Now, we state Chaganty-Sethuraman's theorem for an arbitrary sequence of random variables. Theorem 2.2.3 below obtains an asymptotic expansion for the density function $k_{n}$ of $T_{n} / n$ at the point $m_{n}$ which is in the region of large deviation for $T_{n}$.

Theorem 2.2.3 (Chaganty-Sethuraman). Let $\left\{T_{n}, n \geq 1\right\}$ be a sequence of non-lattice random variables. Let

$$
\phi_{n}(z)=E\left(\exp z T_{n}\right)
$$


and

$$
\psi_{n}(z)=\frac{1}{n} \log \phi_{n}(z)
$$

for a complex number $z$. Denote the interval $(-a, a)$ by $I$ and $\left(-a_{1}, a_{1}\right)$ by $I_{1}$, where $0<a_{1}<a$. Assume that $\phi_{n}(z)$ is nonvanishing and analytic in $\Omega=\{z:|\operatorname{Real}(z)|<a\}$. Let $\left\{m_{n}\right\}$ be a sequence of real numbers. Let

$$
G_{n, \tau}(t)=\psi_{n}(\tau)+i t m_{n}-\psi_{n}(\tau+i t)
$$

for $\tau \in I_{1}$. Assume that $\left\{T_{n}, n \geq 1\right\}$ satisfies the following conditions:

(i') There exists $\beta>0$ such that $\left|\psi_{n}(z)\right|<\beta$ for $z \in \Omega$ and $n \geq 1$.

(ii ') There exist $\alpha>0$ and $\tau_{n} \in I_{1}$ such that $\psi_{n}^{\prime}\left(\tau_{n}\right)=m_{n}$ and $\psi_{n}^{\prime \prime}(\tau) \geq \alpha$, for $\tau \in I_{1}$ and for all $n \geq 1$.

(iii') There exists $\eta>0$ such that for any $0<\delta<\eta$,

$$
\inf _{|t| \geq \delta} \operatorname{Real}\left(G_{n}(t)\right)=\min \left[\operatorname{Real}\left(G_{n}(\delta)\right), \operatorname{Real}\left(G_{n}(-\delta)\right)\right]
$$

for all $n \geq 1$, where $G_{n}(t)=G_{n, \tau_{n}}(t)$.

(iv') There exist $p, l>0$ such that

$$
\sup _{\tau \in I} \int_{-\infty}^{\infty}\left|\frac{\phi_{n}(\tau+i t)}{\phi_{n}(\tau)}\right|^{l / n} d t=O\left(n^{p}\right)
$$


Let $\gamma_{n}(u)=\sup _{s \in I}\left[u s-\psi_{n}(s)\right]$ and $k_{n}$ be the probability density function of $T_{n} / n$. Then

$$
k_{n}\left(m_{n}\right)=\frac{\sqrt{n}}{\sqrt{2 \pi} \sqrt{\psi_{n}^{\prime \prime}\left(\tau_{n}\right)}} \exp \left(-n \gamma_{n}\left(m_{n}\right)\right)[1+O(1 / n)]
$$

The case of lattice random variables is treated in the next theorem.

Theorem 2.2.4 (Chaganty-Sethuraman). Let $\left\{T_{n}, n \geq 1\right\}$ be a sequence of lattice random variables taking values in the set $S=\left\{a_{n}+k h_{n}: k=0, \pm 1, \pm 2, \ldots\right\}$. Define $\phi_{n}(z), \psi_{n}(z)$ as in Theorem 2.2.3. Let $\phi_{n}(z)$ be analytic in $\Omega=$ $\{z:|\operatorname{Real}(z)|<a\}$. Let $\left\{m_{n}=a_{n}+k_{n} h_{n} / n\right\}$ be a sequence of real numbers, where $\left\{k_{n}\right\}$ is a sequence of integers. Suppose that Conditions (i) and (ii ) of Theorem 2.2.3 hold. Further, let us assume the following conditions:

(iii*) There exists $\eta>0$ such that for any $0<\delta<\eta$,

$$
\inf _{\delta \leq|t| \leq \pi /\left|h_{n}\right|} \operatorname{Real}\left(G_{n}(t)\right)=\min \left[\operatorname{Real}\left(G_{n}(\delta)\right), \operatorname{Real}\left(G_{n}(-\delta)\right)\right]
$$

for $n \geq 1$.

(iv $\left.{ }^{*}\right)$ There exist $p, l>0$ such that

$$
\begin{gathered}
\sup _{\tau \in I} \int_{-\pi / h_{n}}^{\pi / h_{n}}\left|\frac{\phi_{n}(\tau+i t)}{\phi_{n}(\tau)}\right|^{l / n} d t=O\left(n^{p}\right) . \\
\text { Let } \gamma_{n}(u)=\sup _{s \in I}\left[u s-\psi_{n}(s)\right] \text { and } \psi_{n}^{\prime}\left(\tau_{n}\right)=m_{n} . \text { Then } \\
\frac{\sqrt{n}}{\left|h_{n}\right|} \operatorname{Pr}\left(T_{n} / n=m_{n}\right)=\frac{1}{\sqrt{2 \pi} \sqrt{\psi_{n}^{\prime \prime}\left(\tau_{n}\right)}} \exp \left(-n \gamma_{n}\left(m_{n}\right)\right)[1+O(1 / n)] .
\end{gathered}
$$


Remark 2.2.5. Condition (iv') of Theorem 2.2.3 is an integrability condition which guarantees the existence of the density function $k_{n}$ of $T_{n} / n$. Conditions $\left(i^{\prime}\right)$ and $\left(i^{\prime}\right)$ together imply that $\left(T_{n}-E\left(T_{n}\right)\right) / \sqrt{\operatorname{var}\left(T_{n}\right)}$ converges in distribution to the standard normal and $\left(T_{n}-E\left(T_{n}\right)\right) / n$ converges to zero in probability. Condition (iii') is satisfied for example if the characteristic functions $\left|\phi_{n}\left(\tau_{n}+i t\right) / \phi_{n}\left(\tau_{n}\right)\right|$ are concave in a neighbourhood of the origin. Verification of condition (iii') in examples poses some difficulties. In Theorem 2.2.8 we show that condition (iii') can be replaced by an easily verifiable condition (iii") and still obtain the conclusion (2.12).

Remark 2.2.6. It is easy to see that Theorem 2.2.3 simplifies to Theorem 2.2.2 when $T_{n}$ is taken to be the sum of $n$ i.i.d. random variables.

Remark 2.2.7. The proofs of Theorems 2.2.2-2.2.4 have the same pattern and they consist of three major steps. In step 1, we use the inversion formula for the characteristic functions of the conjugate distribution (see (2.26) for the definition of a conjugate distribution) to get an expression for the density functions $k_{n}\left(m_{n}\right)$ and $v_{n}\left(m_{n}\right)$. This expression is dependent on the conjugate distribution. We choose the appropriate conjugate distribution using a saddle point technique. We then split the integral $I_{n}$ appearing in the inversion formula into two parts $I_{n 1}$ and $I_{n 2}$ (see (2.31) below). In step 2, we show that $I_{n 1}$ goes to zero exponentially fast and in step 3, we complete the proof by showing that $I_{n 2}=1+O(1 / n)$. 
We now state and outline the proof of Chaganty-Sethuraman's theorem with Condition (iii') replaced by Condition (iii"). We continue to use the same notations introduced earlier.

Theorem 2.2.8. Assume that $\left\{T_{n}, n \geq 1\right\}$ satisfies the following conditions:

(i') There exists $\beta>0$ such that $\left|\psi_{n}(z)\right|<\beta$ for $z \in \Omega$ and $n \geq 1$.

(ii ') There exists $\alpha>0$ and $\tau_{n} \in I_{1}$ such that $\psi_{n}^{\prime}\left(\tau_{n}\right)=m_{n}$ and $\psi_{\pi}^{\prime \prime}(\tau) \geq \alpha$, for $\tau \in I_{1}$ and for all $n \geq 1$.

(iii") Given $\delta>0$ there exists $0<\eta<1$ such that

$$
\limsup \sup _{|t|>\delta}\left|\frac{\phi_{n}\left(\tau_{n}+i t\right)}{\phi_{n}\left(\tau_{n}\right)}\right|^{1 / n}<\eta .
$$

(iv') There exist $p, l>0$ such that

$$
\sup _{\tau \in I} \int_{-\infty}^{\infty}\left|\frac{\phi_{n}(\tau+i t)}{\phi_{n}(\tau)}\right|^{l / n} d t=O\left(n^{p}\right)
$$

Then,

$$
k_{n}\left(m_{n}\right)=\frac{\sqrt{n}}{\sqrt{2 \pi} \sqrt{\psi_{n}^{\prime \prime}\left(\tau_{n}\right)}} \exp \left(-n \gamma_{n}\left(m_{n}\right)\right)[1+O(1 / n)]
$$

We will need the following lemma in the proof of Theorem 2.2.8. 
Lemma 2.2.9. Let $\psi_{n}(z)$ be as defined in (2.8). Assume that the condition (i') of Theorem 2.2.8 holds. Then,

$$
\left|\psi_{n}^{(k)}(\tau)\right| \leq \frac{k ! \beta}{\left(a-a_{1}\right)^{k}}
$$

for $k \geq 1$ and for $\tau \in J_{1}$. Let

$$
R_{n}(\tau+i t)=\psi_{n}(\tau+i t)-\psi_{n}(\tau)-i t \psi_{n}^{\prime}(\tau)-\frac{i t^{2}}{2 !} \psi_{n}^{\prime \prime}(\tau)-\frac{i t^{3}}{3 !} \psi_{n}^{\prime \prime \prime}(\tau)
$$

Then there exists $\delta_{1}>0$ such that for $|t|<\delta_{1}$ and for $\tau \in J_{1}$,

$$
\left|R_{n}(\tau+i t)\right| \leq \frac{2 \beta t^{4}}{\left(a-a_{1}\right)^{4}}
$$

Proof. Since $\psi_{n}$ is analytic in $\Omega$, we can apply Cauchy's theorem for derivatives to get

$$
\psi_{n}^{(k)}(\tau)=\frac{k !}{2 \pi i} \int_{C\left(\tau, a-a_{1}\right)} \frac{\psi_{n}(\omega)}{(\omega-\tau)^{k+1}} d \omega
$$

for $|\tau|<a$ and for all $k \geq 1$ where $C\left(\tau, a-a_{1}\right)=\left\{\omega \in C:|\tau-\omega|=\left(a-a_{1}\right)\right\}$. We use Condition ( $\mathrm{i}^{\prime}$ ) to obtain

$$
\begin{aligned}
\left|\psi_{n}^{(k)}(\tau)\right| & \leq \frac{k !}{2 \pi} \sup _{\omega \in C\left(\tau, a-a_{1}\right)}\left|\psi_{n}(\omega)\right| \int_{C\left(\tau, a-a_{1}\right)} \frac{1}{\left|\omega-\tau_{n}\right|^{k+1}} d \omega \\
& \leq \frac{k ! \beta}{\left(a-a_{1}\right)^{k}}
\end{aligned}
$$

for $k \geq 1$. This proves (2.19). Next, since $\psi_{n}$ is analytic in $\Omega$ and $|\tau|<a$, the following expansion is valid for all $n \geq 1$ and $|t|<\delta_{1}$ where $\delta_{1}<\left(a-a_{1}\right) / 2$,

$$
\psi_{n}(\tau+i t)=\psi_{n}(\tau)+i t \psi_{n}^{\prime}(\tau)+\frac{(i t)^{2}}{2 !} \psi_{n}^{\prime \prime}(\tau)+\frac{(i t)^{3}}{3 !} \psi_{n}^{\prime \prime \prime}(\tau)+R_{n}(\tau+i t)
$$


where

$$
R_{n}(\tau+i t)=\frac{(i t)^{4}}{2 \pi i} \int_{C\left(\tau, a-a_{1}\right)} \frac{\psi_{n}(\omega)}{(\omega-\tau)^{4}(\omega-\tau-i t)} d \omega
$$

An upper bound for $R_{n}$ is given by

$$
\begin{aligned}
\left|R_{n}(\tau+i t)\right| & \leq \frac{t^{4}}{2 \pi} \sup _{\omega \in C\left(\tau, a-a_{1}\right)}\left|\psi_{n}(\omega)\right| \int_{C\left(\tau, a-a_{1}\right)} \frac{1}{|\omega-\tau|^{4}|\omega-\tau-i t|} d \omega \\
& \leq \frac{2 \beta t^{4}}{\left(a-a_{1}\right)^{4}}
\end{aligned}
$$

wherein we have used the relation

$|\omega-\tau-i t| \geq|\omega-\tau|-|t|=\left(a-a_{1}\right)-|t| \geq\left(a-a_{1}\right) / 2$. This proves the lemma.

Next, we outline the proof of Theorem 2.2.8. The proof is given in three major steps.

Proof of Theorem 2.2.8:

Step I. Consider the conjugate distribution $H_{n}$ given by

$$
d H_{n}(x)=\frac{\exp (\tau x)}{\phi_{n}(\tau)} d F_{n}(x)
$$

for $\tau \in I$, where $F_{n}$ denotes the distribution function of $T_{n}$. The characteristic function (c.f.) of the distribution function (d.f.) $H_{n}$ is given by $\phi_{n}(\tau+i t) / \phi_{n}(\tau)$. From (2.17) it follows that $\phi_{n}(\tau+i t) / \phi_{n}(\tau)$ is absolutely integrable. Therefore the p.d.f. of $H_{n}$ exists and it is given by

$$
\frac{1}{2 \pi} \int_{-\infty}^{\infty} \frac{\phi_{n}(\tau+i t)}{\phi_{n}(\tau)} \exp (-i t x) d t
$$


and using (2.26) we get the p.d.f. of $F_{n}$ as

$$
\frac{1}{2 \pi} \int_{-\infty}^{\infty} \phi_{n}(\tau+i t) \exp (-(\tau+i t) x) d t
$$

Thus the p.d.f. of $T_{n} / n$ is given by

$$
\begin{aligned}
k_{n}(x) & =\frac{n}{2 \pi} \int_{-\infty}^{\infty} \phi_{n}(\tau+i t) \exp (-n(\tau+i t) x) d t \\
& =\frac{n}{2 \pi} \int_{-\infty}^{\infty} \exp \left(n\left[\psi_{n}(\tau+i t)-(\tau+i t) x\right]\right) d t
\end{aligned}
$$

The above equality (2.29) is valid for any $\tau \in J_{1}$. The appropriate choice for $\tau$ is $\tau_{n}$ satisfying $\psi_{n}^{\prime}\left(\tau_{n}\right)=m_{n}$. Thus, we have

$$
\begin{aligned}
k_{n}\left(m_{n}\right) & =\frac{n}{2 \pi} \int_{-\infty}^{\infty} \exp \left(n\left[\psi_{n}\left(\tau_{n}+i t\right)-\left(\tau_{n}+i t\right) m_{n}\right]\right) d t \\
& =\left[\frac{n}{2 \pi \psi_{n}^{\prime \prime}\left(\tau_{n}\right)}\right]^{1 / 2} \exp \left(-n \gamma_{n}\left(m_{n}\right)\right) I_{n}
\end{aligned}
$$

where

$$
\begin{aligned}
I_{n}= & {\left[\frac{n \psi_{n}^{\prime \prime}\left(\tau_{n}\right)}{2 \pi}\right]^{1 / 2} \int_{-\infty}^{\infty} \exp \left(n\left[\psi_{n}\left(\tau_{n}+i t\right)-\psi_{n}\left(\tau_{n}\right)-i t m_{n}\right]\right) d t } \\
= & {\left[\frac{n \psi_{n}^{\prime \prime}\left(\tau_{n}\right)}{2 \pi}\right]^{1 / 2}\left[\int_{|t| \geq \delta} \exp \left(n\left[\psi_{n}\left(\tau_{n}+i t\right)-\psi_{n}\left(\tau_{n}\right)-i t m_{n}\right]\right) d t\right.} \\
& \left.+\int_{|t|<\delta} \exp \left(n\left[\psi_{n}\left(\tau_{n}+i t\right)-\psi_{n}\left(\tau_{n}\right)-i t m_{n}\right]\right) d t\right],
\end{aligned}
$$

where $\delta$ is any positive number less than $\delta_{1}$ of Lemma 2.2.9. We choose our $\delta$ small enough such that

$$
M(\delta)=\left[\beta \delta /\left(a-a_{1}\right)^{3}+2 \beta \delta^{2} /\left(a-a_{1}\right)^{4}\right]<\alpha / 2
$$


Thus we can write

$$
I_{n}=I_{n 1}+I_{n 2}(s a y)
$$

We now show that $I_{n 1}$ goes to zero exponentially fast.

Step II. Consider,

$$
\begin{aligned}
\left|I_{n 1}\right| & =\left[\frac{n \psi_{n}^{\prime \prime}\left(\tau_{n}\right)}{2 \pi}\right]^{1 / 2}\left|\int_{|t| \geq \delta} \exp \left(n\left[\psi_{n}\left(\tau_{n}+i t\right)-\psi_{n}\left(\tau_{n}\right)-i t m_{n}\right]\right) d t\right| \\
& \leq\left[\frac{n \psi_{n}^{\prime \prime}\left(\tau_{n}\right)}{2 \pi}\right]^{1 / 2} \int_{|t| \geq \delta}\left|\frac{\phi_{n}\left(\tau_{n}+i t\right)}{\phi_{n}\left(\tau_{n}\right)}\right|^{2} d t \\
& =\left[\frac{n \psi_{n}^{\prime \prime}\left(\tau_{n}\right)}{2 \pi}\right]^{1 / 2} \int_{|t| \geq \delta}\left|\frac{\phi_{n}\left(\tau_{n}+i t\right)}{\phi_{n}\left(\tau_{n}\right)}\right|^{l / n}\left|\frac{\phi_{n}\left(\tau_{n}+i t\right)}{\phi_{n}\left(\tau_{n}\right)}\right|^{(1-l / n)} d t \\
& \leq\left[\frac{n \psi_{n}^{\prime \prime}\left(\tau_{n}\right)}{2 \pi}\right]^{1 / 2}\left[\int_{|t| \geq \delta}\left|\frac{\phi_{n}\left(\tau_{n}+i t\right)}{\phi_{n}\left(\tau_{n}\right)}\right|^{1 / n}\right]_{|t| \geq \delta}^{n(1-l / n)}\left|\frac{\phi_{n}\left(\tau_{n}+i t\right)}{\phi_{n}\left(\tau_{n}\right)}\right|^{l / n} d t \\
& \leq\left[\frac{n \psi_{n}^{\prime \prime}\left(\tau_{n}\right)}{2 \pi}\right]^{1 / 2}\left[\sup _{|t| \geq \delta}\left|\frac{\phi_{n}\left(\tau_{n}+i t\right)}{\phi_{n}\left(\tau_{n}\right)}\right|^{1 / n}\right]^{n(1-l / n)} \int_{-\infty}^{\infty}\left|\frac{\phi_{n}\left(\tau_{n}+i t\right)}{\phi_{n}\left(\tau_{n}\right)}\right|^{l / n} d t
\end{aligned}
$$

From (2.16) and (2.17) it follows that,

$$
\begin{aligned}
\left|I_{n 1}\right| & \leq\left[\frac{n \psi_{n}^{\prime \prime}\left(\tau_{n}\right)}{2 \pi}\right]^{1 / 2} \eta^{n(1-l / n)} O\left(n^{p}\right) \\
& =O\left(n^{p+1 / 2}\right) \exp \left(-\eta_{1}(n-l)\right)
\end{aligned}
$$

where $\eta_{1}=-\log \eta>0$. Thus $I_{n 1}$ goes to zero exponentially fast. Next we will show that $I_{n 2}=1+O(1 / n)$ in step III below.

Step III. Let us recall that 


$$
I_{n 2}=\left[\frac{n \psi_{n}^{\prime \prime}\left(\tau_{n}\right)}{2 \pi}\right]^{1 / 2} \int_{|t|<\delta} \exp \left(n\left[\psi_{n}\left(\tau_{n}+i t\right)-\psi_{n}\left(\tau_{n}\right)-i t m_{n}\right]\right) d t
$$

By making a change of variable $t=s / \sqrt{n}$, we obtain

$$
I_{n 2}=\left[\frac{\psi_{n}^{\prime \prime}\left(\tau_{n}\right)}{2 \pi}\right]^{1 / 2} \int_{|s|<\sqrt{n} \delta} \exp \left(n\left[\psi_{n}\left(\tau_{n}+i s / \sqrt{n}\right)-\psi_{n}\left(\tau_{n}\right)-\frac{i s}{\sqrt{n}} m_{n}\right]\right) d s .
$$

From (2.24) we get,

$$
\begin{gathered}
I_{n 2}=\left[\frac{\psi_{n}^{\prime \prime}\left(\tau_{n}\right)}{2 \pi}\right]^{1 / 2} \int_{|s|<\sqrt{n} \delta} \exp \left(n \left[\frac{-s^{2}}{2 n} \psi_{n}^{\prime \prime}\left(\tau_{n}\right)-\frac{i s^{3}}{6 n \sqrt{n}} \psi_{n}^{\prime \prime \prime}\left(\tau_{n}\right)\right.\right. \\
\left.\left.+R_{n}\left(\tau_{n}+i s / \sqrt{n}\right)\right]\right) d s \\
=\left[\frac{\psi_{n}^{\prime \prime}\left(\tau_{n}\right)}{2 \pi}\right]^{1 / 2} \int_{|s|<\sqrt{n} \delta} \exp \left(-\frac{s^{2}}{2} \psi_{n}^{\prime \prime}\left(\tau_{n}\right)\right)\left[1-\frac{i s^{3}}{6 \sqrt{n}} \psi_{n}^{\prime \prime \prime}\left(\tau_{n}\right)\right. \\
\left.+n R_{n}\left(\tau_{n}+i s / \sqrt{n}\right)+L_{n}(s)\right] d s
\end{gathered}
$$

where

$$
L_{n}(s)=\left[\exp \left(z_{n}\right)-1-z_{n}\right]
$$

and

$$
z_{n}=\left[-\frac{i s^{3}}{6 \sqrt{n}} \psi_{n}^{\prime \prime \prime}\left(\tau_{n}\right)+n R_{n}\left(\tau_{n}+i s / \sqrt{n}\right)\right]
$$

The r.h.s. of (2.37) can be written as the sum of four integrals. The first integral is equal to $1+o(1 / n)$ by Mill's ratio (see Feller (1968) page $175,(1.8))$. It is clear that the second integral is zero. In order to show 
that the third integral is $O(1 / n)$ we will use the bound in (2.21) for $R_{n}$. For that consider,

$$
\begin{aligned}
& \left|n\left[\frac{\psi_{n}^{\prime \prime}\left(\tau_{n}\right)}{2 \pi}\right]^{1 / 2} \int_{|s|<\sqrt{n} \delta} \exp \left(-\frac{s^{2}}{2} \psi_{n}^{\prime \prime}\left(\tau_{n}\right)\right) R_{n}\left(\tau_{n}+i s / \sqrt{n}\right) d s\right| \\
\leq & n\left[\frac{\psi_{n}^{\prime \prime}\left(\tau_{n}\right)}{2 \pi}\right]^{1 / 2} \int_{|s|<\sqrt{n} \delta} \exp \left(-\frac{s^{2}}{2} \psi_{n}^{\prime \prime}\left(\tau_{n}\right)\right)\left|R_{n}\left(\tau_{n}+i s / \sqrt{n}\right)\right| d s \\
\leq & n\left[\frac{\psi_{n}^{\prime \prime}\left(\tau_{n}\right)}{2 \pi}\right]^{1 / 2} \frac{2 \beta}{n^{2}\left(a-a_{1}\right)^{4}} \int_{|s|<\sqrt{n} \delta} s^{4} \exp \left(-\frac{s^{2}}{2} \psi_{n}^{\prime \prime}\left(\tau_{n}\right)\right) d s \\
= & O(1 / n) .
\end{aligned}
$$

Thus

$$
n\left[\frac{\psi_{n}^{\prime \prime}\left(\tau_{n}\right)}{2 \pi}\right]^{1 / 2} \int_{|s|<\sqrt{n} \delta} \exp \left(-\frac{s^{2}}{2} \psi_{n}^{\prime \prime}\left(\tau_{n}\right)\right) R_{n}\left(\tau_{n}+i s / \sqrt{n}\right) d s=O(1 / n)
$$

Finally, to show that the fourth integral is also $O(1 / n)$, we first get an upperbound for $L_{n}(s)$. From (2.19) and (2.21) we have for $|s|<\sqrt{n} \delta$,

$$
\begin{aligned}
\left|z_{n}\right| & =\left|-\frac{i s^{3}}{6 \sqrt{n}} \psi_{n}^{m \prime \prime}\left(\tau_{n}\right)+n R_{n}\left(\tau_{n}+i s / \sqrt{n}\right)\right| \\
& \leq \frac{|s|^{3}}{6 \sqrt{n}}\left|\psi_{n}^{\prime \prime \prime}\left(\tau_{n}\right)\right|+n\left|R_{n}\left(\tau_{n}+i s / \sqrt{n}\right)\right| \\
& \leq\left[\frac{\beta|s|^{3}}{\sqrt{n}\left(a-a_{1}\right)^{3}}+\frac{2 \beta s^{4}}{n\left(a-a_{1}\right)^{4}}\right] \\
& \leq\left[\frac{\beta \delta s^{2}}{\left(a-a_{1}\right)^{3}}+\frac{2 \beta \delta^{2} s^{2}}{\left(a-a_{1}\right)^{4}}\right] \\
& =M(\delta) s^{2}
\end{aligned}
$$


where $M(\delta)=\left[\beta \delta /\left(a-a_{1}\right)^{3}+2 \beta \delta^{2} /\left(a-a_{1}\right)^{4}\right]$. Using the inequality $\left|\exp \left(z_{n}\right)-1-z_{n}\right| \leq\left|z_{n}\right|^{2} \exp \left|z_{n}\right|$ and (2.42) we get

$$
\begin{aligned}
L_{n}(s) & =\left|\exp \left(z_{n}\right)-1-z_{n}\right| \\
& \leq\left[\frac{\beta|s|^{3}}{\sqrt{n}\left(a-a_{1}\right)^{3}}+\frac{2 \beta s^{4}}{n\left(a-a_{1}\right)^{4}}\right]^{2} \exp \left(M(\delta) s^{2}\right) .
\end{aligned}
$$

We will use the bound in (2.43) to show that the fourth integral in the r.h.s. of $(2.37)$ is $O(1 / n)$.

Consider,

$$
\begin{aligned}
& \left|\left[\frac{\psi_{n}^{\prime \prime}\left(\tau_{n}\right)}{2 \pi}\right] \int_{|s|<\sqrt{n} \delta} \exp \left(-\frac{s^{2}}{2} \psi_{n}^{\prime \prime}\left(\tau_{n}\right)\right) L_{n}(s) d s\right| \\
& \leq\left[\frac{\psi_{n}^{\prime \prime}\left(\tau_{n}\right)}{2 \pi}\right] \int_{|s|<\sqrt{n} \delta} \exp \left(-\frac{s^{2}}{2} \psi_{n}^{\prime \prime}\left(\tau_{n}\right)\right) \exp \left(M(\delta) s^{2}\right) \\
& {\left[\frac{\beta|s|^{3}}{\sqrt{n}\left(a-a_{1}\right)^{3}}+\frac{2 \beta s^{4}}{n\left(a-a_{1}\right)^{4}}\right]^{2} d s} \\
& \leq\left[\frac{\psi_{n}^{\prime \prime}\left(\tau_{n}\right)}{2 \pi}\right]^{1 / 2} \int_{|s|<\sqrt{n} \delta} \exp \left(-\left(\frac{\alpha}{2}-M(\delta)\right) s^{2}\right) \\
& {\left[\frac{\beta|s|^{3}}{\sqrt{n}\left(a-a_{1}\right)^{3}}+\frac{2 \beta s^{4}}{n\left(a-a_{1}\right)^{4}}\right]^{2} d s} \\
& =O(1 / n)
\end{aligned}
$$

since we chose $\delta$ such that $M(\delta)<\alpha / 2$. Thus, from (2.37), (2.41), (2.44) it follows that $I_{n 2}=1+O(1 / n)$. The proof of Theorem 2.2 .8 is now complete. 
For lattice random variables $T_{n}$ we have the following analogous theorem.

Theorem 2.2.10. Let $T_{n}$ take values in the set $\left\{a_{n}+k h_{n}: k=0, \pm 1, \pm 2, \ldots\right\}$. Let $\left\{m_{n}=\left(a_{n}+k_{n} h_{n}\right) / n\right\}$ be a sequence of real numbers where $\left\{k_{n}\right\}$ is a sequence of integers. Assume that conditions ( $i^{\prime}$ ) and (ii') of Theorem 2.2.8 hold and replace conditions (iii") and (iv') by the following:

(iii $\left.{ }^{* *}\right)$ Given $\delta>0$, there exists $0<\eta<1$ such that

$$
\limsup \sup _{\delta \leq|t| \leq \pi /\left|h_{n}\right|}\left|\frac{\phi_{n}\left(\tau_{n}+i t\right)}{\phi_{n}\left(\tau_{n}\right)}\right|^{1 / n}<\eta .
$$

$\left(i v^{* *}\right)$ There exist $p, l>0$ such that

$$
\sup _{\tau \in I} \int_{-\pi /\left|h_{n}\right|}^{\pi /\left|h_{n}\right|}\left|\frac{\phi_{n}\left(\tau_{n}+i t\right)}{\phi_{n}\left(\tau_{n}\right)}\right|^{l / n} d t=O\left(n^{p}\right)
$$

Then,

$$
\frac{\sqrt{n}}{\left|h_{n}\right|} \operatorname{Pr}\left(T_{n} / n=m_{n}\right)=\frac{1}{\sqrt{2 \pi} \sqrt{\psi_{n}^{n}\left(\tau_{n}\right)}} \exp \left(-n \gamma_{n}\left(m_{n}\right)\right)[1+O(1 / n)]
$$

Proof. The proof of this theorem is analogous to that of Theorem 2.2.5 except that the range of integration in (2.27) is from $-\pi /\left|h_{n}\right|$ to $\pi /\left|h_{n}\right|$ instead of the whole real line. Hence we skip the details of this proof.

As an application to large deviation local limit theorems for an arbitrary sequence of random variables Chaganty- Sethuraman (1985) have discussed 
two examples in non-parametric theory. One of the examples deals with Wilcoxon signed rank statistic. We show below that the Wilcoxon signed rank statistic satisfies our new Condition $\left(i 2 i^{* *}\right)$.

Example 2.2.11 Let $\left\{X_{n}, n \geq 1\right\}$ be a sequence of i.i.d. random variables with median $m$. Arrange $\left|X_{1}\right|,\left|X_{2}\right|, \ldots,\left|X_{n}\right|$ in increasing order of magnitude and assign ranks $1,2, \ldots, n$. The Wilcoxon signed-ranked statistic $U_{n}$ is defined as the sum of the ranks of positive $X_{i}$ 's. This statistic is used to test the null hypothesis $H_{0}: m=0$ vs $H_{1}: m \neq 0$. Let $T_{n}=U_{n} / n$. The c.f. of $T_{n}$ under the null hypothesis $H_{0}$ is given by

$$
\phi_{n}(z)=\prod_{k=1}^{n}[(\exp (k z / n)+1) / 2], z \in C
$$

and therefore

$$
\psi_{n}(z)=(1 / n) \sum_{k=1}^{n} \log [(\exp (k z / n)+1) / 2], z \in C .
$$

In this example verification of Conditions ( $\left(^{\prime}\right)$, (ii') and $\left(i v^{* *}\right)$ of Theorem 2.2.10 does not pose any difficulties. The analysis in Chaganty and Sethuraman (1985) page 26 shows that, there exists $n_{0}$ and $\delta_{1}>0$ such that for $0<\delta<\delta_{1}$,

$$
\sup _{\delta \leq|t|<\pi / n}\left|\frac{\phi_{n}\left(\tau_{n}+i t\right)}{\phi_{n}\left(\tau_{n}\right)}\right|^{1 / n} \leq \exp \left(-\alpha \delta^{2} / 4\right)
$$

for $n \geq n_{0}$. This verifies Condition $\left(i 2 i^{* *}\right)$. Thus all the conditions of Theorem 2.2.10 are satisfied. Hence, the conclusion of Theorem 2.2.10 yields an expression for $P\left(T_{n}=n m_{n}\right)$. 


\subsection{Large deviation local limit theorems for ratio statistics}

This section deals with the main theorems of this dissertation, namely, Theorems 2.3.1 and 2.3.12. Let $\left\{T_{n}, n \geq 1\right\}$ be an arbitrary sequence of random variables and $\left\{S_{n}, n \geq 1\right\}$ be another arbitrary sequence of positive random variables. Let the two sequences be independent. Theorem 2.3.1 obtains an asymptotic expression for the density function of $R_{n}=T_{n} / S_{n}$ at the point $r_{n}$ in the case where both $T_{n}$ and $S_{n}$ are non-lattice random variables. The other Theorem 2.3.12 obtains similar result in the case where $T_{n}$ is non-lattice and $S_{n}$ is a lattice random variable. We treat the case where $T_{n}$ and $S_{n}$ both are lattice random variables later in Section 2.4.

Our Theorem 2.3.12 simplifies to the result of Chaganty- Sethuraman (see Theorem 2.2.8) in the case where $T_{n}$ is taken to be the sum of $n$ i.i.d. non-lattice random variables and $S_{n}$ is taken to be degenerate at $n$. Thus our theorem generalizes the results obtained by Chaganty and Sethuraman.

The conditions of the Theorems 2.3.1, 2.3.12 are very much simplified when $T_{n}$ and $S_{n}$ are taken to be the sums of i.i.d. random variables. These results are stated in Theorems 2.3.13 and 2.3.14. The conclusion of Theorem 2.3.13 agrees with the heuristic result of Daniels (1954).

Like the theorems in the previous section, the theorems in this section are also obtained under some mild and easily verifiable conditions on the moment generating functions $\phi_{1 n}$ and $\phi_{2 n}$ of $T_{n}$ and $S_{n}$ respectively. These conditions are such that they give bounds on the means of $T_{n} / a_{n}$ 
and $S_{n} / a_{n}$ and variances of $T_{n} / \sqrt{a_{n}}$ and $S_{n} / \sqrt{a_{n}}$ which in turn imply that $T_{n} / a_{n}$ converges to zero in probability and $S_{n} / a_{n}$, in view of (2.54), converges in probability to some positive constant. Thus by Slutsky's theorem $R_{n}=T_{n} / S_{n}$ converges to zero in probability and therefore for any $r_{n}>0$, the asymptotic expressions for the density function of $R_{n}$ at $r_{n}$ obtained in this section become a large deviation local limit theorem. We proceed with some notations.

Let $\left\{T_{n}, n \geq 1\right\}$ be an arbitrary sequence of non-lattice random variables and let $\left\{S_{n}, n \geq 1\right\}$ be a sequence of positive non-lattice valued random variables. Let the two sequences be independent. Let $\phi_{1 n}$ and $\phi_{2 n}$ be the moment generating functions of $\left\{T_{n}, n \geq 1\right\}$ and $\left\{S_{n}, n \geq 1\right\}$ respectively. Let us further assume that $\phi_{1 n}(z)$ is analytic in $\Omega_{1}=\left\{z \in C:|z|<c_{1}\right\}$ and $\phi_{2 n}(z)$ is analytic in $\Omega_{2}=\left\{z \in C:|z|<c_{2}\right\}$ where $C$ denotes the set of all complex numbers and $c_{1}, c_{2}$ are some positive constants. Let $\left\{a_{n}\right\}$ be a sequence of real numbers such that $a_{n} \rightarrow \infty$.

Let

$$
\begin{aligned}
& \psi_{1 n}(z)=\frac{1}{a_{n}} \log \phi_{1 n}(z), z \in \Omega_{1} . \\
& \psi_{2 n}(z)=\frac{1}{a_{n}} \log \phi_{2 n}(z), z \in \Omega_{2} .
\end{aligned}
$$

Let $J_{1}=\left(-b_{1}, b_{1}\right), 0<b_{1}<c_{1}$ and $J_{2}=\left(-b_{2}, b_{2}\right), 0<b_{2}<c_{2}$. Let $\left\{r_{n}\right\}$ be a bounded sequence of real numbers such that there exists a sequence $\left\{\tau_{n}\right\}$ contained in $J_{1}$ satisfying

$$
\psi_{1 n}^{\prime}\left(\tau_{n}\right)-r_{n} \psi_{2 n}^{\prime}\left(-r_{n} \tau_{n}\right)=0
$$

for $r_{n} \tau_{n} \in J_{2}$ for all $n \geq 1$. Let there exist positive constants $\alpha_{1}$ and $\alpha_{2}$ 
such that

$$
\psi_{1 n}^{\prime \prime}(\tau)>\alpha_{1} \text { and } \psi_{2 n}^{\prime}\left(\tau^{\prime}\right)>\alpha_{2}
$$

and $\tau^{\prime} \in J_{2}$.

We now state the main theorem of this section. Theorem 2.3.1 below obtains a large deviation local limit theorem for the ratio statistic $R_{n}=T_{n} / S_{n}$ at the point $r_{n}$.

Theorem 2.3.1. Assume that the two sequences $\left\{T_{n}, n \geq 1\right\}$ and $\left\{S_{n}, n \geq 1\right\}$ satisfy the following conditions:

(A1) There exist $\beta_{1}<\infty$ and $\beta_{2}<\infty$ such that

$$
\left|\psi_{1 n}(z)\right|<\beta_{1} \text { for } z \in \Omega_{1}
$$

and

$$
\left|\psi_{2 n}(z)\right|<\beta_{2} \text { for } z \in \Omega_{2}, \text { for all } n \geq 1
$$

(A2) For any given $\delta>0$, there exist $0<\eta<1$ and $q>0$ such that

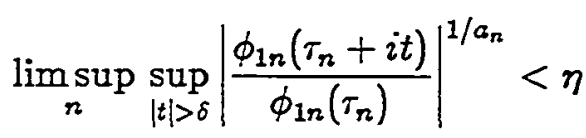

and

$$
\sup _{|t|>\delta}\left|\psi_{2 r_{n}}^{\prime}\left(-r_{n}\left(\tau_{n}+i t\right)\right)\right|=O\left(a_{r_{n}}^{q}\right)
$$

(AS) There exist $p, l>0$ such that

$$
\sup _{\tau \in J_{1}} \int_{-\infty}^{\infty}\left|\frac{\phi_{1 n}(\tau+i t)}{\phi_{1 n}(\tau)}\right|^{l / a_{n}} d t=O\left(a_{n}^{p}\right) .
$$


Then an asymptotic expansion for the density function $g_{n}$ of $T_{n} / S_{n}$ at the point $r_{n}$ is given by

$$
\begin{aligned}
g_{n}\left(r_{n}\right)= & \frac{\sqrt{a_{n}} \psi_{2 n}^{\prime}\left(-r_{n} \tau_{n}\right)}{\left.\left[2 \pi\left[\psi_{1 n}^{\prime \prime}\left(\tau_{n}\right)+r_{n}^{2} \psi_{2 n}^{\prime \prime}\left(-r_{n} \tau_{n}\right)\right]\right]\right]^{1 / 2}} \\
& \quad \times \exp \left(a_{n}\left[\psi_{1 n}\left(\tau_{n}\right)+\psi_{2 n}\left(-r_{n} \tau_{n}\right)\right]\right)\left[1+O\left(1 / a_{n}\right)\right] .(2
\end{aligned}
$$

The proof of this theorem is deferred until the end of Lemma 2.3.11. We now make some remarks about the Conditions (A1), (A2) and (A3) of the above theorem.

Remark 2.3.2. Condition (A1) of Theorem 2.3.1 requires that $\psi_{1 \text { n }}$ and $\psi_{2 n}$ be bounded uniformly in $n$ in a circle around the origin in the complex plane. In Lemma 2.3.7 we show that Condition(A1) implies that the means of $T_{n} / a_{n}$ and $S_{n} / a_{n}$ are bounded and the variances of $T_{n} / \sqrt{a_{n}}$ and $S_{n} / \sqrt{a_{n}}$ are also bounded. Applying Chebyshev's inequality we can verify that $\left(T_{n}-E\left(T_{n}\right)\right) / a_{n} \rightarrow 0$ in probability and $\left(S_{n}-E\left(S_{n}\right)\right) / a_{n} \rightarrow c$ in probability for some $c$ positive. The application of Slutsky's theorem shows that $R_{n}=$ $T_{n} / S_{n}$ goes to zero in probability.

Remark 2.3.3. Since $\left|\phi_{1 n}\left(\tau_{n}+i t\right) / \phi_{1 n}\left(\tau_{n}\right)\right|$ is the characteristic function of a non-lattice random variable, for each $\delta>0$ we can always find $0<\eta_{n}<1$ such that

$$
\sup _{|t|>\delta}\left|\phi_{1 n}\left(\tau_{n}+i t\right) / \phi_{1 n}\left(\tau_{n}\right)\right|^{1 / a_{n}}<\eta_{n}
$$


Condition (A2) requires that the limsup $r_{n}=\eta<1$. We use Condition (A2) mainly in Lemma 2.3.9 to show that the term $I_{n 1}$ defined in (2.68) goes exponentially fast to zero.

Remark 2.3.4. Condition (A3) guarantees the existence of the density function of $T_{n}$ and permits the use of inversion formula to get an expression for the p.d.f. of $T_{n}$. This condition is also used to show that the term $I_{n 1}$ in (2.68) goes exponentially fast to zero.

Remark 2.3.5. It is interesting to note that the conclusion of the Theorem 2.3.1 remains the same if $\phi_{1 n}$ is replaced by $\phi_{2 n}$ in (2.57) and (2.59) of Conditions (A2) and (A3).

Remark 2.3.6. Selection of an appropriate sequence $\left\{r_{n}\right\}$ of real numbers guarantees the existence of a saddle point $\left(\tau_{n}, 0\right)$ for the function $\operatorname{Real}\left(\psi_{1 n}(\tau+i t)+\psi_{2 n}\left(-r_{n}(\tau+i t)\right)\right.$ for each $n$. We use this saddle point to get an asymptotic expression for the integral in (2.107).

We will need the following Lemmas 2.3.7 thru 2.3.10 in the proof of Theorem 2.3.1. Lemma 2.3.7 obtains bounds which are independent of $\mathrm{n}$ for $\psi_{1 n}^{(k)}$ and $\psi_{2 n}^{(k)}$ for $k \geq 1$ and also for the remainder terms $R_{1 n}(\tau+i t)$ and $R_{2 n}(\tau+i t)$ defined below.

Lemma 2.3.7. Let $\psi_{1 n}$ and $\psi_{2 n}$ be as defined in (2.51) and (2.52). Assume that Condition (A1) of Theorem 2.3.1 holds. Then the following holds:

$$
\sup _{\tau \in J_{j}}\left|\psi_{j n}^{(k)}(\tau)\right| \leq \frac{k ! \beta_{j}}{\left(c_{j}-b_{j}\right)^{k}}
$$


for $j=1,2$ and for $k \geq 1$. Let

$$
R_{j n}(\tau+i t)=\psi_{j n}(\tau+i t)-\psi_{j n}(\tau)-(i t) \psi_{j n}^{\prime}(\tau)-\frac{(i t)^{2}}{2 !} \psi_{j n}^{\prime \prime}(\tau)-\frac{(i t)^{3}}{3 !} \psi_{j n}^{\prime \prime \prime}(\tau)
$$

for $\tau \in J_{j}, j=1,2$. Then there exists $\delta_{j}<\left(c_{j}-b_{j}\right) / 2$ such that

$$
\sup _{\tau \in J_{j}}\left|R_{j n}(\tau+i t)\right| \leq \frac{2 \beta_{j} t^{4}}{\left(c_{j}-b_{j}\right)^{4}}
$$

for $|t|<\delta_{j}$ and for $j=1,2$.

Proof. The proof of this lemma is similar to the proof of Lemma 2.2.9 and hence it is omitted.

Remark 2.3.8. Let $\sup \left|r_{n}\right|=r$. Let $\delta_{3}=\delta_{2} / r$. Since $\psi_{2 n}^{t}(z)$ is analytic in $\Omega_{2}$ the following expansion is valid for all $n \geq 1$ and $|t|<\delta_{3}$ :

$$
\begin{gathered}
\psi_{2 n}^{\prime}\left(-r_{n}\left(\tau_{n}+i t\right)\right)=\psi_{2 n}^{\prime}\left(-r_{n} \tau_{n}\right)+\left(-i r_{n} t\right) \psi_{2 n}^{\prime \prime}\left(-r_{n} \tau_{n}\right)+\frac{\left(-i r_{n} t\right)^{2}}{2 !} \psi_{2 n}^{\prime \prime \prime}\left(-r_{n} \tau_{n}\right) \\
+R\left(-\tau_{n}\left(\tau_{n}+i t\right)\right)
\end{gathered}
$$

where

$$
R\left(-r_{n}\left(\tau_{n}+i t\right)\right)=\frac{\left(-i r_{n} t\right)^{3}}{2 \pi i} \int_{C_{2}} \frac{\psi_{2 n}^{\prime}(\omega)}{\left(\omega-\left(-r_{n} \tau_{n}\right)\right)^{3}\left(\omega-\left(-r_{n} \tau_{n}\right)+i r_{n} \dot{\tau}\right)} d \omega
$$

and $C_{2}=C\left(-r_{n} \tau_{n}, c_{2}-b_{2}\right)$. Therefore, for $|t|<\delta_{3}$ we have,

$$
\left|R\left(-\tau_{n}\left(\tau_{n}+i t\right)\right)\right| \leq \frac{2 \beta_{2} r^{3} t^{3}}{\left(c_{2}-b_{2}\right)^{3}}
$$


Lemma 2.3.9 shows that the term $I_{n 1}$ (see (2.113)) appearing in the proof of Theorem 2.3.1 goes exponentially fast to zero.

Lemma 2.3.9. Assume that the Conditions (AE) and (AS) of Theorem 2.8.1 are satisfied. Let

$$
f_{n}(z)=\psi_{1 n}(z)+\psi_{2 n}\left(-r_{n} z\right)
$$

Then

$$
\begin{gathered}
I_{n 1}=\left[\frac{a_{n} f_{n}^{\prime \prime}\left(\tau_{n}\right)}{2 \pi}\right]^{1 / 2} \int_{|t| \geq \delta} \exp \left(\left[a_{n}\left(f_{n}\left(\tau_{n}+i t\right)-f_{n}\left(\tau_{n}\right)\right)\right]\right) \\
\times \frac{\psi_{2 n}^{\prime}\left(-r_{n}\left(\tau_{n}+i t\right)\right)}{\psi_{2 n}^{\prime}\left(-r_{n} \tau_{n}\right)} d t
\end{gathered}
$$

goes exponentially fast to zero for all $\delta>0$ such that $\delta<\min \left(\delta_{1}, \delta_{2}, \delta_{3}\right)$ where $\delta_{1}$ and $\delta_{2}$ are as in Lemma 2.3.7 and $\delta_{3}$ is as in Remark 2.S.8.

Proof: Consider

$$
\begin{aligned}
\left|I_{n 1}\right| & =\left|\left[\frac{a_{n} f_{n}^{\prime \prime}\left(\tau_{n}\right)}{2 \pi}\right]^{1 / 2} \int_{|t| \geq \delta} \exp \left(\left[a_{n}\left(f_{n}\left(\tau_{n}+i t\right)-f_{n}\left(\tau_{n}\right)\right)\right]\right) \frac{\psi_{2 n}^{\prime}\left(-r_{n}\left(\tau_{n}+i t\right)\right)}{\psi_{2 n}^{\prime}\left(-r_{n} \tau_{n}\right)} d t\right| \\
& \leq\left[\frac{a_{n} f_{n}^{\prime \prime}\left(\tau_{n}\right)}{2 \pi}\right]^{1 / 2} \int_{|t| \geq \delta}\left|\exp \left(\left[a_{n}\left(f_{n}\left(\tau_{n}+i t\right)-f_{n}\left(\tau_{n}\right)\right)\right]\right)\right| \frac{\psi_{2 n}^{\prime}\left(-r_{n}\left(\tau_{n}+i t\right)\right)}{\psi_{2 n}^{\prime}\left(-r_{n} \tau_{n}\right)} \mid d t
\end{aligned}
$$

Replacing $f_{n}\left(\tau_{n}\right)$ by $\psi_{1 n}\left(\tau_{n}\right)+\psi_{2 n}\left(-r_{n} \tau_{n}\right)$ inside the integral, we get

$$
\left|I_{n I}\right| \leq\left[\frac{a_{n} f_{n}^{\prime \prime}\left(\tau_{n}\right)}{2 \pi}\right]^{1 / 2} \int_{|t| \geq \delta}\left|\frac{\phi_{1 n}\left(\tau_{n}+i t\right)}{\phi_{1 n}\left(\tau_{n}\right)} \frac{\phi_{2 n}\left(-r_{n}\left(\tau_{n}+i t\right)\right)}{\phi_{2 n}\left(-r_{n} \tau_{n}\right)}\right|\left|\frac{\psi_{2 n}^{\prime}\left(-r_{n}\left(\tau_{n}+i t\right)\right)}{\psi_{2 n}^{\prime}\left(-r_{n} \tau_{n}\right)}\right| d t
$$




$$
\begin{gathered}
\leq\left[\frac{a_{n} f_{n}^{\prime \prime}\left(\tau_{n}\right)}{2 \pi}\right]^{1 / 2} \int_{|t| \geq \delta}\left|\frac{\phi_{1 n}\left(\tau_{n}+i t\right)}{\phi_{1 n}\left(\tau_{n}\right)}\right|\left|\frac{\psi_{2 n}^{\prime}\left(-r_{n}\left(\tau_{n}+i t\right)\right)}{\psi_{2 n}^{\prime}\left(-r_{n} \tau_{n}\right)}\right| d t \\
\leq\left[\frac{a_{n} f_{n}^{\prime \prime}\left(\tau_{n}\right)}{2 \pi}\right]^{1 / 2} \sup _{|t| \geq \delta}\left|\frac{\psi_{2 n}^{\prime}\left(-r_{n}\left(\tau_{n}+i t\right)\right)}{\psi_{2 n}^{\prime}\left(-r_{n} \tau_{n}\right)}\right| \\
\int_{|t| \geq \delta}\left|\frac{\phi_{1 n}\left(\tau_{n}+i t\right)}{\phi_{1 n}\left(\tau_{n}\right)}\right|^{l / a_{n}}\left|\frac{\phi_{1 n}\left(\tau_{n}+i t\right)}{\phi_{1 n}\left(\tau_{n}\right)}\right|^{1-l / a_{n}} d t .
\end{gathered}
$$

Using the fact that $\psi_{2 n}^{\prime}(\tau)>\alpha_{2}$, for $\tau \in J_{2}$ and Condition (A2) we get,

$$
\left|I_{n 1}\right| \leq\left[\frac{a_{n} f_{n}^{\prime \prime}\left(\tau_{n}\right)}{2 \pi}\right]^{1 / 2} \frac{1}{\alpha_{2}} O\left(a_{n}^{q}\right) \eta^{a_{n}\left(1-l / a_{n}\right)} \int_{-\infty}^{\infty}\left|\frac{\phi_{1 n}\left(\tau_{n}+i t\right)}{\phi_{1 n}\left(\tau_{n}\right)}\right|^{l / a_{n}} d t .
$$

Finally, Condition (A3) implies that,

$$
\left|I_{n 1}\right| \leq O\left(a_{n}^{p+q+1 / 2}\right) \exp \left(-\eta_{1}\left(a_{n}-l\right)\right)
$$

where $\eta_{1}=-\log \eta>0$. Hence $I_{n 1}$ goes exponentially fast to zero.

We need the following lemma to prove Lemma 2.3.11.

Lemma 2.3.10. Let $\psi_{1 n}$ and $\psi_{2 n}$ be as defined in (2.51) and (2.52). Assume that Condition (A1) of Theorem 2.3.1 holds.

Let

$$
d_{n}=\psi_{1 n}^{\prime \prime}\left(\tau_{n}\right)+\tau_{n}^{2} \psi_{2 n}^{\prime \prime}\left(-\tau_{n} \tau_{n}\right)
$$

and

$$
L_{n}(s)=\left[\exp \left(z_{n}\right) \frac{\psi_{2 n}^{\prime}\left(-r_{n}\left(\tau_{n}+i \frac{s}{\sqrt{a_{n}}}\right)\right)}{\psi_{2 n}^{\prime}\left(-r_{n} \tau_{n}\right)}-1-z_{n}\right]
$$


where

$$
\begin{aligned}
z_{n}= & {\left[\frac{-i s^{3}}{6 \sqrt{a_{n}}} \psi_{1 n}^{\prime \prime \prime}\left(\tau_{n}\right)+\frac{i r_{n}^{3} s^{3}}{6 \sqrt{a_{n}}} \psi_{2 n}^{\prime \prime \prime}\left(-r_{n} \tau_{n}\right)\right.} \\
& +a_{n} R_{1 n}\left(\tau_{n}+i \frac{s}{\sqrt{a_{n}}}\right)+a_{n} R_{2 n}\left(-r_{n}\left(\tau_{n}+i \frac{s}{\sqrt{a_{n}}}\right)\right]
\end{aligned}
$$

Then there exists $\delta>0$ such that

$$
\left[\frac{d_{n}}{2 \pi}\right]^{1 / 2} \int_{|s|<\sqrt{a_{n}} \delta} \exp \left(\frac{-s^{2}}{2} d_{n}\right) L_{n}(s) d s=O\left(1 / a_{n}\right)
$$

Proof. Let $\delta$ be as in Lemma 2.3.9. Then

$$
Q_{n}=\left[\frac{d_{n}}{2 \pi}\right]^{1 / 2} \int_{|s|<\sqrt{a_{n}} \delta} \exp \left(\frac{-s^{2}}{2} d_{n}\right)\left[\exp \left(z_{n}\right) \frac{\psi_{2 n}^{\prime}\left(-\tau_{n}\left(\tau_{n}+i \frac{s}{\sqrt{a_{n}}}\right)\right)}{\psi_{2 n}^{\prime}\left(-r_{n} \tau_{n}\right)}-1-z_{n}\right] d s
$$

We can write $Q_{n}$ as the sum of four integrals as follows:

$$
\begin{aligned}
Q_{n}= & {\left[\frac{d_{n}}{2 \pi}\right]^{1 / 2} \int_{|s|<\sqrt{a_{n}} \delta} \exp \left(\frac{-s^{2}}{2} \dot{d}_{n}\right)\left[\exp \left(\left(z_{n}\right)-1-z_{n}\right) \frac{\psi_{2 n}^{\prime}\left(-r_{n}\left(\tau_{n}+i \frac{s}{\sqrt{a_{n}}}\right)\right)}{\psi_{2 n}^{\prime}\left(-r_{n} \tau_{n}\right)}\right.} \\
& \left.+\left(\frac{\psi_{2 n}^{\prime}\left(-\tau_{n}\left(\tau_{n}+i \frac{s}{\sqrt{a_{n}}}\right)\right)}{\psi_{2 n}^{\prime}\left(-r_{n} \tau_{n}\right)}-1\right)-\left(z_{n}\right)+\left(z_{n} \frac{\psi_{2 n}^{\prime}\left(-\tau_{n}\left(\tau_{n}+i \frac{s}{\sqrt{a_{n}}}\right)\right)}{\psi_{2 n}^{\prime}\left(-r_{n} \tau_{n}\right)}\right)\right] d s \\
= & I_{n 1}^{\prime}+I_{n 2}^{\prime}+I_{n 3}^{\prime}+I_{n 4}^{\prime}(s a y)
\end{aligned}
$$

where

$$
I_{n 1}^{\prime}=\left[\frac{d_{n}}{2 \pi}\right]^{1 / 2} \int_{|s|<\sqrt{a_{n}} \delta} \exp \left(\frac{-s^{2}}{2} d_{n}\right) \exp \left(\left(z_{n}\right)-1-z_{n}\right) \frac{\psi_{2 n}^{\prime}\left(-r_{n}\left(\tau_{n}+i \frac{s}{\sqrt{a_{n}}}\right)\right)}{\psi_{2 n}^{\prime}\left(-r_{n} \tau_{n}\right)} d s
$$




$$
\begin{gathered}
I_{n 2}^{\prime}=\left[\frac{d_{n}}{2 \pi}\right]^{1 / 2} \int_{|s|<\sqrt{a_{n}} \delta} \exp \left(\frac{-s^{2}}{2} d_{n}\right)\left(\frac{\psi_{2 n}^{\prime}\left(-r_{n}\left(\tau_{n}+i \frac{s}{\sqrt{a_{n}}}\right)\right)}{\psi_{2 n}^{\prime}\left(-r_{n} \tau_{n}\right)}-1\right) d s \\
I_{n 3=}^{\prime}=\left[\frac{d_{n}}{2 \pi}\right]^{1 / 2} \int_{|s|<\sqrt{a_{n}} \delta} \exp \left(\frac{-s^{2}}{2} d_{n}\right)\left(-z_{n}\right) d s \\
I_{n 4}^{\prime}=\left[\frac{d_{n}}{2 \pi}\right]^{1 / 2} \int_{|s|<\sqrt{a_{n}} \delta} \exp \left(\frac{-s^{2}}{2} d_{n}\right) z_{n} \frac{\psi_{2 n}^{\prime}\left(-r_{n}\left(\tau_{n}+i \frac{s}{\sqrt{a_{n}}}\right)\right)}{\psi_{2 n}^{\prime}\left(-r_{n} \tau_{n}\right)} d s .
\end{gathered}
$$

The proof is completed by showing that $I_{n i}^{\prime}=O\left(1 / a_{n}\right)$ for $i=1,2,3,4$. In order to show that $I_{n 1}^{\prime}=O\left(1 / a_{n}\right)$, we get an upper bound for $\left(\exp \left(z_{n}\right)-\right.$ $1-z_{n}$ ), by first obtaining an upperbound for $z_{n}$. For $|s|<\sqrt{a_{n}} \delta$, using Condition (A1) and (2.61) we get that

$$
\begin{aligned}
\left|z_{n}\right| & \leq \frac{|s|^{3} \beta_{1}}{\sqrt{a_{n}}\left(c_{1}-b_{1}\right)^{3}}+\frac{\left|r_{n}\right|^{3}|s|^{3} \beta_{2}}{\sqrt{a_{n}}\left(c_{2}-b_{2}\right)^{3}}+\frac{2 \beta_{1} s^{4}}{a_{n}\left(c_{1}-b_{1}\right)^{4}}+\frac{2 \beta_{2} r_{n}^{4} s^{4}}{a_{n}\left(c_{2}-b_{2}\right)^{4}} \\
& \leq \frac{\beta_{1} \delta|s|^{2}}{\left(c_{1}-b_{1}\right)^{3}}+\frac{\beta_{2}\left|r_{n}\right|^{3} \delta|s|^{2}}{\left(c_{2}-b_{2}\right)^{3}}+\frac{2 \beta_{1} \delta^{2} s^{2}}{\left(c_{1}-b_{1}\right)^{4}}+\frac{2 \beta_{2} r_{n}^{4} \delta^{2} s^{2}}{\left(c_{2}-b_{2}\right)^{4}} \\
& =\left[\frac{\beta_{1} \delta}{\left(c_{1}-b_{1}\right)^{3}}+\frac{\beta_{2}\left|r_{n}\right|^{3} \delta}{\left(c_{2}-b_{2}\right)^{3}}+\frac{2 \beta_{1} \delta^{2}}{\left(c_{1}-b_{1}\right)^{4}}+\frac{2 \beta_{2} r_{n}^{4} \delta^{2}}{\left(c_{2}-b_{2}\right)^{4}}\right] s^{2}
\end{aligned}
$$

Using the boundedness of $r_{n}$ in (2.82), we get

$$
\left|z_{n}\right| \leq M(\delta) s^{2}
$$

where

$$
\begin{aligned}
M(\delta)= & {\left[\beta_{1} \delta /\left(c_{1}-b_{1}\right)^{3}+\beta_{2} r^{3} \delta /\left(c_{2}-b_{2}\right)^{3}\right.} \\
& \left.+2 \beta_{1} \delta^{2} /\left(c_{1}-b_{1}\right)^{4}+2 \beta_{2} r^{4} \delta^{2} /\left(c_{2}-b_{2}\right)^{4}\right]
\end{aligned}
$$


We choose our $\delta$ such that $M(\delta)<\alpha_{1} / 2$. Using the simple inequality $\left|\exp \left(z_{n}\right)-1-z_{n}\right| \leq\left|z_{n}\right|^{2} \exp \left(\left|z_{n}\right|\right)$ we obtain,

$$
\begin{aligned}
\left|\exp \left(z_{n_{2}}\right)-1-z_{n}\right| \leq & {\left[\frac{|s|^{3} \beta_{1}}{\sqrt{a_{n}}\left(c_{1}-b_{1}\right)^{3}}+\frac{r^{3}|s|^{3} \beta_{2}}{\sqrt{a_{n}}\left(c_{2}-b_{2}\right)^{3}}\right.} \\
& \left.+\frac{2 \beta_{1} s^{4}}{a_{n}\left(c_{1}-b_{1}\right)^{4}}+\frac{2 \beta_{2} r^{4} s^{4}}{a_{n}\left(c_{2}-b_{2}\right)^{4}}\right]^{2} \exp \left(M(\delta) s^{2}\right) .
\end{aligned}
$$

We are now in a position to show that $I_{n 1}^{\prime}=O\left(1 / a_{n}\right)$. Consider

$$
\begin{aligned}
\left|I_{n 1}^{\prime}\right|= & \left|\left[\frac{d_{n}}{2 \pi}\right]^{1 / 2} \int_{|s|<\sqrt{a_{n}} \delta} \exp \left(\frac{-s^{2}}{2} d_{n}\right)\left(\exp \left(z_{n}\right)-1-z_{n}\right) \frac{\psi_{2 n}^{\prime}\left(-r_{n}\left(\tau_{n}+i \frac{s}{\sqrt{a_{n}}}\right)\right)}{\psi_{2 n}^{\prime}\left(-r_{n} \tau_{n}\right)} d s\right| \\
\leq & {\left[\frac{d_{n}}{2 \pi}\right]^{1 / 2} \int_{|s|<\sqrt{a_{n}} \delta} \exp \left(\frac{-s^{2}}{2} d_{n}\right)\left|\left(\exp \left(z_{n}\right)-1-z_{n}\right)\right|\left|\frac{\psi_{2 n}^{\prime}\left(-r_{n}\left(\tau_{n}+i \frac{s}{\sqrt{a_{n}}}\right)\right)}{\psi_{2 n}^{\prime}\left(-r_{n} \tau_{n}\right)}\right| d s } \\
\leq & {\left[\frac{d_{n}}{2 \pi}\right]^{1 / 2} \int_{|s|<\sqrt{a_{n}} \delta} \exp \left(\frac{-s^{2}}{2} d_{n}\right) \exp \left(M(\delta) s^{2}\right)\left[\frac{|s|^{3} \beta_{1}}{\sqrt{a_{n}}\left(c_{1}-b_{1}\right)^{3}}+\frac{r^{3}|s|^{3} \beta_{2}}{\sqrt{a_{n}}\left(c_{2}-b_{2}\right)^{3}}\right.} \\
& \left.+\frac{2 \beta_{1} s^{4}}{a_{n}\left(c_{1}-b_{1}\right)^{4}}+\frac{2 \beta_{2} \tau^{4} s^{4}}{a_{n}\left(c_{2}-b_{2}\right)^{4}}\right]^{2}\left|\frac{\psi_{2 n}^{\prime}\left(-r_{n}\left(\tau_{n}+i \frac{s}{\sqrt{a_{n}}}\right)\right)}{\psi_{2 n}^{\prime}\left(-r_{n} \tau_{n}\right)}\right| d s \\
\leq & \frac{1}{a_{n}}\left[\frac{d_{n}}{2 \pi}\right]^{1 / 2} \int_{|s|<\sqrt{a_{n} \delta}} \exp \left(\frac{-s^{2}}{2}\left(\alpha_{1}-2 M(\delta)\right)\right)\left[\frac{\beta_{1}|s|^{3}}{\left(c_{1}-b_{1}\right)^{3}}+\frac{r^{3}|s|^{3} \beta_{2}}{\left(c_{2}-b_{2}\right)^{3}}\right. \\
& \left.+\frac{2 \beta_{1} s^{4}}{\sqrt{a_{n}}\left(c_{1}-b_{1}\right)^{4}}+\frac{2 \beta_{2} \tau^{4} s^{4}}{\sqrt{a_{n}}\left(c_{2}-b_{2}\right)^{4}}\right]^{2}\left|\frac{\psi_{2 n}^{\prime}\left(-r_{n}\left(\tau_{n}+i \frac{s}{\sqrt{a_{n}}}\right)\right)}{\psi_{2 n}^{\prime}\left(-r_{n} \tau_{n}\right)}\right| d s .
\end{aligned}
$$

Using Taylor series expansion for $\psi_{2 n}^{\prime}$ and the bounds (2.61) and (2.63) obtained in Lemma 2.3.7 we can show that $\left|\psi_{2 n}^{\prime}\left(-r_{n}\left(\tau_{n}+i \frac{s}{\sqrt{a_{n}}}\right)\right) / \psi_{2 n}^{\prime}\left(-r_{n} \tau_{n}\right)\right|$ is bounded in $n$. Since $M(\delta)<\alpha_{1} / 2$ we get that $I_{n 1}^{\prime}=O\left(1 / a_{n}\right)$. Thus

$$
I_{n 1}^{\prime}=O\left(1 / a_{n}\right)
$$

\section{2-38}


Next we show that $I_{n 2}^{\prime}=O\left(1 / a_{n}\right)$. Consider

$$
\begin{aligned}
I_{n 2}^{\prime}= & {\left[\frac{d_{n}}{2 \pi}\right]^{1 / 2} \int_{|s|<\sqrt{a_{n}} \delta} \exp \left(\frac{-s^{2}}{2} d_{n}\right)\left(\frac{\psi_{2 n}^{\prime}\left(-r_{n}\left(\tau_{n}+i \frac{s}{\sqrt{a_{n}}}\right)\right)}{\psi_{2 n}^{\prime}\left(-r_{n} \tau_{n}\right)}-1\right) d s } \\
= & {\left[\frac{d_{n}}{2 \pi}\right]^{1 / 2} \int_{|s|<\sqrt{a_{n} \delta}} \exp \left(\frac{-s^{2}}{2} d_{n}\right)\left[-i r_{n} \frac{s}{\sqrt{a_{n}}} \frac{\psi_{2 n}^{\prime \prime}\left(-\tau_{n} \tau_{n}\right)}{\psi_{2 n}^{\prime}\left(-\tau_{n} \tau_{n}\right)}\right.} \\
& \left.-r_{n}^{2} \frac{s^{2}}{a_{n}} \frac{\psi_{2 n}^{\prime \prime \prime}\left(-r_{n} \tau_{n}\right)}{\psi_{2 n}^{\prime}\left(-r_{n} \tau_{n}\right)}+\frac{R\left(-r_{n} \tau_{n}-i r_{n} \frac{s}{\sqrt{a_{n}}}\right)}{\psi_{2 n}^{\prime}\left(-r_{n} \tau_{n}\right)}\right] d s
\end{aligned}
$$

where $R\left(-r_{n}\left(\tau_{n}+i t\right)\right)$ is as given in (2.65). It is easy to see that the first integral on the right hand side of $I_{n 2}^{\prime}$ is zero. The use of bound on $\psi_{2 n}^{\prime \prime \prime}\left(-\tau_{n} \tau_{n}\right)$ (see (2.61)) and the fact that $\psi_{2 n}^{\prime}\left(-r_{n} \tau_{n}\right)>\alpha_{2}$, it follows that the second integral is $O\left(1 / a_{n}\right)$. Similarly (2.66) and the fact that $\psi_{2 n}^{\prime}\left(-r_{n} \tau_{n}\right)>\alpha_{2}$ imply that the third integral is $O\left(1 / a_{n}^{2}\right)$. Therefore,

$$
I_{n 2}^{\prime}=O\left(1 / a_{n}\right)
$$

Next, consider

$$
\begin{aligned}
I_{n 3}^{\prime}= & {\left[\frac{d_{n}}{2 \pi}\right]^{1 / 2} \int_{|s|<\sqrt{a_{n}} \delta} \exp \left(\frac{-s^{2}}{2} d_{n}\right)\left(-z_{n}\right) d s } \\
= & (-1)\left[\frac{d_{n}}{2 \pi}\right]^{1 / 2} \int_{|s|<\sqrt{a_{n}} \delta} \exp \left(\frac{-s^{2}}{2} d_{n}\right)\left[\frac{-i s^{3}}{6 \sqrt{a_{n}}} \psi_{1 n}^{\prime \prime \prime}\left(\tau_{n}\right)+\frac{i r_{n}^{3} s^{3}}{6 \sqrt{a_{n}}} \psi_{2 n}^{\prime \prime \prime}\left(-r_{n} \tau_{n}\right)\right. \\
& \left.+a_{n} R_{1 n}\left(\tau_{n}+i \frac{s}{\sqrt{a_{n}}}\right)+a_{n} R_{2 n}\left(-r_{n}\left(\tau_{n}+i \frac{s}{\sqrt{a_{n}}}\right)\right)\right]
\end{aligned}
$$

The first two integrals on the right hand side of $I_{n 3}^{\prime}$ are zero and the third and fourth integrals using (2.63) can be seen to be equal to $O\left(1 / a_{n}\right)$. 
Thus

$$
I_{n 3}^{\prime}=O\left(1 / a_{n}\right)
$$

Lastly, consider

$$
\begin{aligned}
I_{n 4}^{\prime}= & {\left[\frac{d_{n}}{2 \pi}\right]^{1 / 2} \int_{|s|<\sqrt{a_{n}} \delta} \exp \left(\frac{-s^{2}}{2} d_{n}\right) z_{n} \frac{\psi_{2 n}^{\prime}\left(-r_{n}\left(\tau_{n}+i \frac{s}{\sqrt{a_{n}}}\right)\right)}{\psi_{2 n}^{\prime}\left(-r_{n} \tau_{n}\right)} d s } \\
= & {\left[\frac{d_{n}}{2 \pi}\right]^{1 / 2} \int_{|s|<\sqrt{a_{n}} \delta} \exp \left(\frac{-s^{2}}{2} d_{n}\right)\left[\frac{-i s^{3}}{6 \sqrt{a_{n}}} \psi_{1 n}^{\prime \prime \prime}\left(\tau_{n}\right)+\frac{i r_{n}^{3} s^{3}}{6 \sqrt{a_{n}}} \psi_{2 n}^{\prime \prime \prime}\left(-\tau_{n} \tau_{n}\right)\right.} \\
& \left.+a_{n} R_{1 n}\left(\tau_{n}+i \frac{s}{\sqrt{a_{n}}}\right)+a_{n} R_{2 n}\left(-\tau_{n}\left(\tau_{n}+i \frac{s}{\sqrt{a_{n}}}\right)\right)\right]\left[1-i r_{n} \frac{s}{\sqrt{a_{n}}} \frac{\psi_{2 n}^{\prime \prime}\left(-r_{n} \tau_{n}\right)}{\psi_{2 n}^{\prime}\left(-r_{n} \tau_{n}\right)}\right. \\
& \left.-r_{n}^{2} \frac{s^{2}}{a_{n}} \frac{\psi_{2 n}^{\prime \prime \prime}\left(-r_{n} \tau_{n}\right)}{\psi_{2 n}^{\prime}\left(-r_{n} \tau_{n}\right)}+\frac{R\left(-r_{n} \tau_{n}-i r_{n} \frac{s}{\sqrt{a_{n}}}\right)}{\psi_{2 n}^{\prime}\left(-r_{n} \tau_{n}\right)}\right] d s .
\end{aligned}
$$

$I_{n 4}^{\prime}$ can be easily shown to be $O\left(1 / a_{n}\right)$. Therefore, it follows from $(2.86)$, (2.88), (2.90) and (2.91) that $Q_{n}=O\left(1 / a_{n}\right)$. This completes the proof of the lemma.

The next lemma shows that the term $I_{n 2}$ appearing in the proof of the main Theorem 2.3.1, (see (2.111)) is $1+O\left(1 / a_{n}\right)$.

Lemma 2.3.11. Let $f_{n}(t)$ be as defined in (2.67). Assume that Conditions (A1) and (A2) of Theorem 2.3.1 hold. Let $\delta$ be as in Lemma 2.3.10. Then

$$
\begin{aligned}
I_{n 2} & =\left[\frac{a_{n} d_{n}}{2 \pi}\right]^{1 / 2} \int_{|t|<\delta} \exp \left(a_{n}\left[f_{n}\left(\tau_{n}+i t\right)-f_{n}\left(\tau_{n}\right)\right]\right) \frac{\psi_{2 n}^{\prime}\left(-r_{n}\left(\tau_{n}+i t\right)\right)}{\psi_{2 n}^{\prime}\left(-r_{n} \tau_{n}\right)} d t \\
& =1+O\left(1 / a_{n}\right) .
\end{aligned}
$$


Proof. Consider

$$
\begin{gathered}
I_{n 2}=\left[\frac{a_{n} d_{n}}{2 \pi}\right]^{1 / 2} \int_{|t|<\delta} \exp \left(a _ { n } \left[\psi_{1 n}\left(\tau_{n}+i t\right)-\psi_{1 n}\left(\tau_{n}\right)+\psi_{2 n}\left(-r_{n}\left(\tau_{n}+i t\right)\right)\right.\right. \\
\left.\left.-\psi_{2 n}\left(-r_{n} \tau_{n}\right)\right]\right) \frac{\psi_{2 n}^{\prime}\left(-r_{n}\left(\tau_{n}+i t\right)\right)}{\psi_{2 n}^{\prime}\left(-\tau_{n} \tau_{n}\right)} d t
\end{gathered}
$$

Making a change of variable $t=s / \sqrt{a_{n}}$, we get,

$$
\begin{aligned}
I_{n 2}=\left[\frac{d_{n}}{2 \pi}\right]^{1 / 2} & \int_{|s|<\sqrt{a_{n}} \delta} \exp \left(a _ { n } \left[\psi_{1 n}\left(\tau_{n}+i \frac{s}{\sqrt{a_{n}}}\right)-\psi_{1 n}\left(\tau_{n}\right)\right.\right. \\
& \left.\left.+\psi_{2 n}\left(-r_{n}\left(\tau_{n}+i \frac{s}{\sqrt{a_{n}}}\right)\right)-\psi_{2 n}\left(-r_{n} \tau_{n}\right)\right]\right) \frac{\psi_{2 n}^{\prime}\left(-r_{n}\left(\tau_{n}+i \frac{s}{\sqrt{a_{n}}}\right)\right)}{\psi_{2 n}^{\prime}\left(-r_{n} \tau_{n}\right)} d s .
\end{aligned}
$$

Using the Taylor's series expansions for $\psi_{1 n}$ and $\psi_{2 n}$ (see (2.62)), we get

$$
\begin{aligned}
I_{n 2}= & {\left[\frac{d_{n}}{2 \pi}\right]^{1 / 2} \int_{|s|<\sqrt{a_{n}} \delta} \exp \left(-a_{n}\left[\frac{s^{2}}{2 a_{n}} \psi_{1 n}^{\prime \prime}\left(\tau_{n}\right)+\frac{r_{n}^{2} s^{2}}{2 a_{n}} \psi_{2 n}^{\prime \prime}\left(-r_{n} \tau_{n}\right)\right.\right.} \\
& +\frac{i s^{3}}{6 a_{n} \sqrt{a_{n}}} \psi_{1 n}^{\prime \prime \prime}\left(\tau_{n}\right)-\frac{i r_{n}^{3} s^{3}}{6 a_{n} \sqrt{a_{n}}} \psi_{2 n}^{\prime \prime \prime}\left(-r_{n} \tau_{n}\right)-R_{1 n}\left(\tau_{n}+i \frac{s}{\sqrt{a_{n}}}\right) \\
& \left.\left.-R_{2 n}\left(-r_{n}\left(\tau_{n}+i \frac{s}{\sqrt{a_{n}}}\right)\right)\right]\right) \frac{\psi_{2 n}^{\prime}\left(-r_{n}\left(\tau_{n}+i \frac{s}{\sqrt{a_{n}}}\right)\right)}{\psi_{2 n}^{\prime}\left(-r_{n} \tau_{n}\right)} d s \\
& \quad \int_{|s|<\sqrt{a_{n} \delta}} \exp \left(\frac{-s^{2}}{2 \pi}\left[\psi_{1 n}^{\prime \prime}\left(\tau_{n}\right)+\tau_{n}^{2} \psi_{2 n}^{\prime \prime}\left(-r_{n} \tau_{n}\right)\right]\right) \\
& \times \exp \left(\frac{-i s^{3}}{6 \sqrt{a_{n}}} \psi_{1 n}^{\prime \prime \prime}\left(\tau_{n}\right)+\frac{i r_{n}^{3} s^{3}}{6 \sqrt{a_{n}}} \psi_{2 n}^{\prime \prime \prime}\left(-r_{n} \tau_{n}\right)+a_{n} R_{1 n}\left(\tau_{n}+i \frac{s}{\sqrt{a_{n}}}\right)\right. \\
& \left.+a_{n} R_{2 n}\left(-r_{n}\left(\tau_{n}+i \frac{s}{\sqrt{a_{n}}}\right)\right)\right) \frac{\psi_{2 n}^{\prime}\left(-r_{n}\left(\tau_{n}+i \frac{s}{\sqrt{a_{n}}}\right)\right)}{\psi_{2 n}^{\prime}\left(-r_{n} \tau_{n}\right)} d s .
\end{aligned}
$$


Noting that $\psi_{1_{n}}^{\prime \prime}\left(\tau_{n}\right)+r_{n}^{2} \psi_{2_{n}}^{\prime \prime}\left(-r_{n} \tau_{n}\right)$ is $d_{n}$ defined in (2.72), we get that

$$
\begin{gathered}
I_{n 2}=\left[\frac{d_{n}}{2 \pi}\right]^{1 / 2} \int_{|s|<\sqrt{a_{n}} \delta} \exp \left(\frac{-d_{n} s^{2}}{2}\right)\left[1-\frac{-i s^{3}}{6 \sqrt{a_{n}}} \psi_{1 n}^{\prime \prime \prime}\left(\tau_{n}\right)+\frac{i r_{n}^{3} s^{3}}{6 \sqrt{a_{n}}} \psi_{2 n}^{\prime \prime \prime}\left(-\tau_{n} \tau_{n}\right)\right. \\
\left.+a_{n} R_{1 n}\left(\tau_{n}+i \frac{s}{\sqrt{a_{n}}}\right)+a_{n} R_{2 n}\left(-r_{n}\left(\tau_{n}+i \frac{s}{\sqrt{a_{n}}}\right)\right)+L_{n}(s)\right] d s
\end{gathered}
$$

where $d_{n}, z_{n}$ and $L_{n}(s)$ are as defined in (2.72), (2.74) and (2.73) respectively. The right hand side of (2.96) involves six integrals. Let us analyze these integrals.

The $1^{\text {st }}$ integral

$$
\begin{aligned}
{\left[\frac{d_{n}}{2 \pi}\right]^{1 / 2} \int_{|s|<\sqrt{a_{n}} \delta} \exp \left(\frac{-s^{2}}{2} d_{n}\right) d s } & =1-2 \Phi\left(-\sqrt{a_{n}} \delta \sqrt{d_{n}}\right) \\
& =1+o\left(1 / a_{n}\right)
\end{aligned}
$$

follows from Mill's Ratio (see Feller (1968) page 175,(1.8)).

The $2^{\text {nd }}$ integral

$$
\left[\frac{d_{n}}{2 \pi}\right]^{1 / 2} \frac{i \psi_{1 n}^{\prime \prime}\left(\tau_{n}\right)}{6 \sqrt{a_{n}}} \int_{|s|<\sqrt{a_{n}} \delta} s^{3} \exp \left(\frac{-s^{2}}{2} d_{n}\right) d s=0 .
$$

The $3^{\text {rd }}$ integral

$$
\left[\frac{d_{n}}{2 \pi}\right]^{1 / 2} \frac{i r_{n}^{3} \psi_{1 n}^{\prime \prime}\left(-r_{n} \tau_{n}\right)}{6 \sqrt{a_{n}}} \int_{|s|<\sqrt{a_{n}} \delta} s^{3} \exp \left(\frac{-s^{2}}{2} d_{n}\right) d s=0 .
$$

For the $4^{\text {th }}$ integral consider

$$
\left|\left[\frac{d_{n}}{2 \pi}\right]^{1 / 2} \int_{|s|<\sqrt{c_{n}} \delta} a_{n} R_{1 n}\left(\tau_{n}+i \frac{s}{\sqrt{a_{n}}}\right) \exp \left(\frac{-s^{2}}{2} d_{n}\right) d s\right|
$$




$$
\begin{aligned}
& \leq a_{n}\left[\frac{d_{n}}{2 \pi}\right]^{1 / 2} \int_{|s|<\sqrt{a_{n}} \delta} \exp \left(\frac{-s^{2}}{2} d_{n}\right)\left|R_{1 n}\left(\tau_{n}+i \frac{s}{\sqrt{a_{n}}}\right)\right| d s \\
& \leq \frac{1}{a_{n}}\left[\frac{d_{n}}{2 \pi}\right]^{1 / 2} \frac{2 \beta_{1}}{\left(c_{1}-b_{1}\right)^{4}} \int_{|s|<\sqrt{a_{n}} \delta} s^{4} \exp \left(\frac{-s^{2}}{2} d_{n}\right) d s \\
& =O\left(1 / a_{n}\right) .
\end{aligned}
$$

Thus

$$
\left[\frac{d_{n}}{2 \pi}\right]^{1 / 2} \int_{|s|<\sqrt{a_{n}} \delta} a_{n} R_{1 n}\left(\tau_{n}+i \frac{s}{\sqrt{a_{n}}}\right) \exp \left(\frac{-s^{2}}{2} d_{n}\right) d s=O\left(1 / a_{n}\right) .
$$

Similarly, the $5^{\text {th }}$ integral can be shown to be equal to $O\left(1 / a_{n}\right)$, that is,

$$
\begin{aligned}
& {\left[\frac{d_{n}}{2 \pi}\right]^{1 / 2} \int_{|s|<\sqrt{a_{n}} \delta} a_{n} R_{2 n}\left(-r_{n}\left(\tau_{n}+i \frac{s}{\sqrt{a_{n}}}\right)\right) \exp \left(\frac{-s^{2}}{2} d_{n}\right) d s } \\
= & O\left(1 / a_{n}\right) .
\end{aligned}
$$

The $6^{\text {th }}$ integral

$$
\left[\frac{d_{n}}{2 \pi}\right]^{1 / 2} \int_{|s|<\sqrt{a_{n}} \delta} \exp \left(\frac{-s^{2}}{2} d_{n}\right) L_{n}(s) d s
$$

is nothing but $Q_{n}$ defined by (2.76) and therefore, by Lemma 2.3.10 it is equal to $O\left(1 / a_{n}\right)$. Thus

$$
I_{n 2}=1+O\left(1 / a_{n}\right)
$$

This completes the proof of Lemma 2.3.11.

We now proceed with the proof of the main Theorem 2.3.1. 
Proof. Let $G_{n}$ be the distribution function of $R_{n}=T_{n} / S_{n}$. Then

$$
G_{n}(r)=P\left[\frac{T_{n}}{S_{n}} \leq r\right]=\int_{0}^{\infty} F_{1 n}(r y) d F_{2 n}(y)
$$

The density function, $g_{n}$, of $G_{n}$ is given by

$$
g_{n}(r)=\int_{0}^{\infty} y f_{1 n}(r y) d F_{2 n}(y)
$$

where $f_{1 n}$ is the p.d.f. of $T_{n}$.

Using the conjugate distribution technique (see (2.26), (2.27) and (2.28)), we get that

$$
f_{1 n}(r y)=\frac{1}{2 \pi} \int_{-\infty}^{\infty} \phi_{1 n}(\tau+i t) \exp (-(\tau+i t) r y) d t
$$

which is true for all $\tau \in J_{1}$. Later we will choose $\tau$ appropriately. Substituting (2.105) in (2.104) we obtain

$$
\begin{aligned}
g_{n}(r) & =\int_{0}^{\infty} y\left[\frac{1}{2 \pi} \int_{-\infty}^{\infty} \phi_{1 n}(\tau+i t) \exp (-(\tau+i t) r y) d t\right] d F_{2 n}(y) \\
& =\frac{1}{2 \pi} \int_{-\infty}^{\infty} \phi_{1 n}(\tau+i t)\left[\int_{0}^{\infty} y \exp (-(\tau+i t) r y) d F_{2 n}(y)\right] d t \\
& =\frac{1}{2 \pi} \int_{-\infty}^{\infty} \phi_{1 n}(\tau+i t) \phi_{2 n}^{\prime}(-r(\tau+i t)) d t \\
& =\frac{a_{n}}{2 \pi} \int_{-\infty}^{\infty} \exp \left(a_{n}\left[\psi_{1 n}(\tau+i t)+\psi_{2 n}(-r(\tau+i t))\right]\right) \psi_{2 n}^{\prime}(-r(\tau+i t)) d t
\end{aligned}
$$

Saddle point technique suggests that when $r$ is replaced by $r_{n}$ an appropriate choice of $\tau$ is $\tau_{n}$ where $\tau_{n}$ is such that

$$
\psi_{1 n}^{\prime}\left(\tau_{n}\right)-r_{n} \psi_{2 n}^{\prime}\left(-r_{n} \tau_{n}\right)=0
$$

$2-44$ 
for $\tau_{n} \in J_{1}$ and $r_{n} \tau_{n} \in J_{2}$. Therefore, we have,

$$
\begin{aligned}
g_{n}\left(r_{n}\right) & =\frac{a_{n}}{2 \pi} \int_{-\infty}^{\infty} \exp \left(a_{n}\left[\psi_{1 n}\left(\tau_{n}+i t\right)+\psi_{2 n}\left(-r_{n}\left(\tau_{n}+i t\right)\right)\right]\right) \psi_{2 n}^{\prime}\left(-r_{n}\left(\tau_{n}+i t\right)\right) d t \\
& =\frac{\sqrt{a_{n}} \psi_{2 n}^{\prime}\left(-r_{n} \tau_{n}\right) \exp \left(a_{n}\left[\psi_{1 n}\left(\tau_{n}\right)+\psi_{2 n}\left(-r_{n} \tau_{n}\right)\right]\right)}{\left[2 \pi\left[\psi_{1 n}^{\prime \prime}\left(\tau_{n}\right)+r_{n}^{2} \psi_{2 n}^{\prime \prime}\left(-r_{n} \tau_{n}\right)\right]\right]^{1 / 2}} I_{n}
\end{aligned}
$$

where

$$
\begin{aligned}
I_{n}= & {\left[\frac{a_{n}\left[\psi_{1 n}^{\prime \prime}\left(\tau_{n}\right)+r_{n}^{2} \psi_{2 n}^{n}\left(-\tau_{n} \tau_{n}\right)\right]}{2 \pi}\right]^{1 / 2} \int_{-\infty}^{\infty} \exp \left(a _ { n } \left[\psi_{1 n}\left(\tau_{n}+i t\right)-\psi_{1 n}\left(\tau_{n}\right)\right.\right.} \\
& \left.\left.+\psi_{2 n}\left(-r_{n}\left(\tau_{n}+i t\right)\right)-\psi_{2 n}\left(-r_{n} \tau_{n}\right)\right]\right) \frac{\psi_{2 n}^{\prime}\left(-r_{n}\left(\tau_{n}+i t\right)\right)}{\psi_{2 n}^{\prime}\left(-\tau_{n} \tau_{n}\right)} d t . \quad(2.109)
\end{aligned}
$$

Let $f_{n}(z)$ and $d_{n}$ be as defined in (2.67) and (2.72) respectively. We have,

$$
\begin{aligned}
I_{n}= & {\left[\frac{a_{n} d_{n}}{2 \pi}\right]^{1 / 2} \int_{-\infty}^{\infty} \exp \left(a_{n}\left[f_{n}\left(\tau_{n}+i t\right)-f_{n}\left(\tau_{n}\right)\right]\right) \frac{\psi_{2 n}^{\prime}\left(-r_{n}\left(\tau_{n}+i t\right)\right)}{\psi_{2 n}^{\prime}\left(-\tau_{n} \tau_{n}\right)} d t } \\
= & {\left[\frac{a_{n} d_{n}}{2 \pi}\right]^{1 / 2}\left[\int_{|t| \geq \delta} \exp \left(a_{n}\left[f_{n}\left(\tau_{n}+i t\right)-f_{n}\left(\tau_{n}\right)\right]\right) \frac{\psi_{2 n}^{\prime}\left(-r_{n}\left(\tau_{n}+i t\right)\right)}{\psi_{2 n}^{\prime}\left(-\tau_{n} \tau_{n}\right)} d t\right.} \\
& \left.+\int_{|t|<\delta} \exp \left(a_{n}\left[f_{n}\left(\tau_{n}+i t\right)-f_{n}\left(\tau_{n}\right)\right]\right) \frac{\psi_{2 n}^{\prime}\left(-r_{n}\left(\tau_{n}+i t\right)\right)}{\psi_{2 n}^{\prime}\left(-r_{n} \tau_{n}\right)} d t\right]
\end{aligned}
$$

where $\delta$ is chosen such that $\delta<\min \left(\delta_{1}, \delta_{2}, \delta_{3}\right)$ and $M(\delta)<\alpha_{1} / 2$ where $\delta_{1}$, $\delta_{2}$ are as in Lemma 2.3.7 and $\delta_{3}$ is as in Remark 2.3.8 and

$$
\begin{aligned}
M(\delta)= & {\left[\beta_{1} \delta /\left(c_{1}-b_{1}\right)^{3}+\beta_{2} r^{3} \delta /\left(c_{2}-b_{2}\right)^{3}\right.} \\
& \left.+2 \beta_{1} \delta^{2} /\left(c_{1}-b_{1}\right)^{4}+2 \beta_{2} r^{2} \delta^{2} /\left(c_{2}-b_{2}\right)^{4}\right] .
\end{aligned}
$$

Thus

$$
I_{n}=I_{n 1}+I_{n 2}
$$


where

$$
I_{n 1}=\left[\frac{a_{n} d_{n}}{2 \pi}\right]^{1 / 2} \int_{|t| \geq \delta} \exp \left(a_{n}\left[f_{n}\left(\tau_{n}+i t\right)-f_{n}\left(\tau_{n}\right)\right]\right) \frac{\psi_{2 n}^{\prime}\left(-r_{n}\left(\tau_{n}+i t\right)\right)}{\psi_{2 n}^{\prime}\left(-r_{n} \tau_{n}\right)} d t
$$

and

$$
I_{n 2}=\left[\frac{a_{n} d_{n}}{2 \pi}\right]^{1 / 2} \int_{|t|<\delta} \exp \left(a_{n}\left[f_{n}\left(\tau_{n}+i t\right)-f_{n}\left(\tau_{n}\right)\right]\right) \frac{\psi_{2 n}^{\prime}\left(-r_{n}\left(\tau_{n}+i t\right)\right)}{\psi_{2 n}^{\prime}\left(-r_{n} \tau_{n}\right)} d t .
$$

Lemma 2.3.9 shows that the term $I_{n 1}$ goes exponentially fast to zero and Lemma 2.3.11 shows that $I_{n 2}=1+O\left(1 / a_{n}\right)$.

Thus

$$
I_{n}=1+O\left(1 / a_{n}\right)
$$

Hence the proof of the theorem is complete.

Next, we obtain similar asymptotic expression for the density function of ratio statistic $R_{n}=T_{n} / S_{n}$ for the case where $\left\{T_{n}, n \geq 1\right\}$ is a sequence of non-lattice random variables and $\left\{S_{n}, n \geq 1\right\}$ is a sequence of positive lattice random variables. This result is stated below in Theorem 2.3.12.

Theorem 2.3.12. Let $\left\{T_{n}, n \geq 1\right\}$ be a sequence of non-latice random variables with distribution functions $F_{1 n}$ and let $\left\{S_{n}, n \geq 1\right\}$ be a sequence of positive lattice random variables with distribution functions $\vec{F}_{2 n}$. Let the two sequences $\left\{T_{n}, n \geq 1\right\}$ and $\left\{S_{n}, n \geq 1\right\}$ be independent. Let $S_{n}$ take values in the set $S=\left\{a_{n}+k h_{n}: a_{n}\right.$ and $h_{n}$ are such that $\left.a_{n}+k h_{n}>0\right\}$. Let $\psi_{1 n}$ and $\psi_{2 n}$ be as defined in (2.51) and (2.52) respectively. Assume that (2.53) is satisfied for an appropriate sequence $r_{n}$. Let $\left\{T_{n}\right\}$ and $\left\{S_{n}\right\}$ further 
satisfy the Conditions (A1), (A2) and (AS) of Theorem 2.3.1. Then, an asymptotic expression for the p.d.f., $g_{2 n}$, of $T_{n} / S_{n}$ at the point $r_{n}$ is given by

$$
\begin{aligned}
g_{2 n}\left(r_{n}\right)= & \frac{a_{n} \psi_{2 n}^{\prime}\left(-r_{n} \tau_{n}\right)}{\left[2 \pi a_{n}\left[\psi_{1 n}^{\prime \prime}\left(\tau_{n}\right)+r_{n}^{2} \psi_{2 n}^{\prime \prime}\left(-r_{n} \tau_{n}\right)\right]\right]^{1 / 2}} \\
& \quad \exp \left(a_{n}\left[\psi_{1 n}\left(\tau_{n}\right)+\psi_{2 n}\left(-r_{n} \tau_{n}\right)\right]\right)\left[1+O\left(1 / a_{n}\right)\right]
\end{aligned}
$$

The proof of this theorem runs parallel to that of earlier Theorems 2.3.1. Hence, we will give first few steps of the proof of this theorem. Proof. Let $G_{2 n}$ be the distribution function of $T_{n} / S_{n}$. Then,

$$
\begin{aligned}
G_{2 n}(r) & =P\left(\frac{T_{n}}{S_{n}} \leq r\right) \\
& =\sum_{y \in S} P\left(T_{n} \leq r y\right) P\left(S_{n}=y\right) \\
& =\sum_{y \in S} F_{1 n}(r y) P\left(S_{n}=y\right) .
\end{aligned}
$$

Therefore the p.d.f. of $T_{n} / S_{n}$ is given by

$$
\begin{aligned}
g_{2 n}(r) & =\sum_{y \in S} y f_{1 n}(r y) P\left(S_{n}=y\right) \\
& =\sum_{y \in S} y P\left(S_{n}=y\right) f_{1 n}(r y) .
\end{aligned}
$$

Using the inversion formula for the conjugate distribution as in (2.109) we can get an expression for $f_{1 n}(r y)$. Thus we have

$$
g_{2 n}(r)=\sum_{y \in S} y P\left(S_{n}=y\right)\left[\frac{1}{2 \pi} \int_{-\infty}^{\infty} \phi_{1 n}(\tau+i t) \exp (-(\tau+i t) r y) d t\right]
$$




$$
\begin{aligned}
& =\frac{1}{2 \pi} \int_{-\infty}^{\infty} \phi_{1 n}(\tau+i t)\left[\sum_{y \in S} y P\left(S_{n}=y\right) \exp (-(\tau+i t) r y)\right] d t \\
& =\frac{1}{2 \pi} \int_{-\infty}^{\infty} \phi_{1 n}(\tau+i t) \phi_{2 n}^{\prime}(-r(\tau+i t)) d t \\
& =\frac{a_{n}}{2 \pi} \int_{-\infty}^{\infty} \exp \left(a_{n}\left[\psi_{1 n}(\tau+i t)+\psi_{2 n}(-r(\tau+i t))\right]\right) \psi_{2 n}^{\prime}(-r(\tau+i t)) d t
\end{aligned}
$$

Replacing $r$ by $r_{n}$ and $\tau$ by $\tau_{n}$ we get

$$
g_{2 n}\left(r_{n}\right)=\frac{a_{n}}{2 \pi} \int_{-\infty}^{\infty} \exp \left(a_{n}\left[\psi_{1 n}\left(\tau_{n}+i t\right)+\psi_{2 n}\left(-r_{n}\left(\tau_{n}+i t\right)\right)\right]\right) \psi_{2 n}^{\prime}\left(-r_{n}\left(\tau_{n}+i t\right)\right) d t
$$

The rest of the proof is similar to that of Theorem 2.3.1. Hence

$$
\begin{aligned}
g_{2 n}\left(r_{n}\right)= & \frac{a_{n} \psi_{2 n}^{\prime}\left(-r_{n} \tau_{n}\right)}{\left[2 \pi a_{n}\left[\psi_{1 n}^{\prime \prime}\left(\tau_{n}\right)+r_{n}^{2} \psi_{2 n}^{\prime \prime}\left(-r_{n} \tau_{n}\right)\right]\right]^{1 / 2}} \\
& \times \exp \left(a_{n}\left[\psi_{1 n}\left(\tau_{n}\right)+\psi_{2 n}\left(-r_{n} \tau_{n}\right)\right]\right)\left[1+O\left(1 / a_{n}\right)\right]
\end{aligned}
$$

Theorems 2.3.13 and 2.3.14 below obtain asymptotic expressions for the density of the ratio of sums of i.i.d. random variables. Theorem 2.3 .13 deals with the occurance of non-lattice $T_{n}$ and non-lattice $S_{n}$ where as Theorem 2.3.14 deals with the occurance of non-lattice $T_{n}$ and lattice $S_{n}$. When $T_{n}$ and $S_{n}$ are sums of $n$ i.i.d. random variables not only that the conditions of Theorems 2.3.13 and 2.3.14 become simpler, but, part 1 of condition (A2) $((2.57))$ is automatically satisfied. We skip the proofs of these theorems as they can easily be deduced from Theoremas 2.3.1 and 2.3.12. 
Theorem 2.3.13. Let $\left\{X_{n}, n \geq 1\right\}$ and $\left\{Y_{n}>0, n \geq 1\right\}$ be two sequences of non-lattice random variables with distribution functions $F_{1}$ and $F_{2}$ respectively. Let the two sequences be independent. Let $\phi_{1}$ and $\phi_{2}$ denote the characteristic functions of $\left\{X_{n}, n \geq 1\right\}$ and $\left\{Y_{n}>0, n \geq 1\right\}$ respectively. Let $\psi_{i}(z)=\log \phi_{i}(z)$ for $i=1,2$. Let $\phi_{i}(z)$ be non-vanishing and analytic in $\Omega_{i}=\left\{z \in C:|z|<c_{i}\right\}$ for $i=1,2$. Let $J_{i}$ denote the interval $\left(-b_{i}, b_{i}\right)$ where $0<b_{i}<c_{i}$ for $i=1,2$. Further, let $\left\{r_{n}\right\}$ be a sequence of real numbers such that there exists $\left\{\tau_{n}\right\} \in J_{1}$ and

$$
\psi_{1}^{\prime}\left(\tau_{n}\right)-r_{n} \psi_{2}^{\prime}\left(-r_{n} \tau_{n}\right)=0
$$

for $\tau_{n} \tau_{n} \in J_{2}$. Let there exist $\alpha_{1}>0$ and $\alpha_{2}>0$ such that $\psi_{1}^{\prime \prime}(\tau)>\alpha_{1}$ for $\tau \in J_{1}$ and $\psi_{2}^{\prime}(\tau)>\alpha_{2}$ for $\tau \in J_{2}$.

Assume the following conditions:

(B1) There exist $\beta_{i}<\infty$ such that

$$
\left|\psi_{i}(z)\right|<\beta_{i} \text { for all } z \in J_{i} \text { for } i=1,2 \text {. }
$$

(B2) Given $\delta>0$, there exists $q>0$, such that

$$
\sup _{|t|>\delta}\left|\psi_{2}^{\prime}\left(-r_{n}\left(\tau_{n}+i t\right)\right)\right|=O\left(n^{q}\right) .
$$

(BS) There exists $B<\infty$ such that

$$
\operatorname{sip}_{\tau \in J_{1}} \int_{-\infty}^{\infty}\left|\frac{\phi_{1}(\tau+i t)}{\phi_{1}(\tau)}\right|<B .
$$

Let $q_{1 n}$ denote the probability density function of $T_{n} / S_{n}$ where

$$
\begin{array}{r}
T_{n}=X_{1}+\cdots+X_{n} \text { and } S_{n}=Y_{1}+\cdots+Y_{n} . \text { Then } \\
q_{1 n}\left(r_{n}\right)=\frac{\sqrt{n} \psi_{2}^{\prime}\left(-r_{n} \tau_{n}\right)}{\left[2 \pi\left[\psi_{1}^{\prime \prime}\left(\tau_{n}\right)+r_{n}^{2} \psi_{2}^{\prime \prime}\left(-r_{n} \tau_{n}\right)\right]\right]^{1 / 2}}
\end{array}
$$




$$
\times \exp \left(n\left[\psi_{1}\left(\tau_{n}\right)+\psi_{2}\left(-\tau_{n} \tau_{n}\right)\right]\right)[1+O(1 / n)]
$$

Theorem 2.3.14. Let $\left\{X_{n}, n \geq 1\right\}$ be a sequence of non-lattice random variables with common distribution function $F_{1}$ and let $\left\{Y_{n}>0, n \geq 1\right\}$ be a sequence of lattice valued random variables with common distribution function $F_{2}$. Let the two sequnces be independent. Let $\phi_{1}$ and $\phi_{2}$ denote the characteristic functions of $\left\{X_{n}, n \geq 1\right\}$ and $\left\{Y_{n}>0, n \geq 1\right\}$ respectively. Let $\phi_{i}(z)$ be non- vanishing and analytic in $\Omega_{i}=\left\{z \in C:|z|<c_{i}\right\}$ for $i=1,2$. Let $J_{i}$ denote the interval $\left(-b_{i}, b_{i}\right)$ where $0<b_{i}<c_{i}$ for $i=1,2$. Further, let $\left\{r_{n}\right\}$ be a sequence of real numbers such that there exists $\left\{\tau_{n}\right\} \in J_{1}, r_{n} \tau_{n} \in J_{2}$ and

$$
\psi_{1}^{\prime}\left(\tau_{n}\right)-r_{n} \psi_{2}^{\prime}\left(-r_{n} \tau_{n}\right)=0
$$

Also, let there exist $\alpha_{1}>0$ such that $\psi_{1}^{\prime \prime}(\tau)>\alpha_{1}$.

Assume Conditions (B1), (B2) and (BS) of Theorem 2.3.18. Let $q_{2 n}$ denote the probability density function of $T_{n} / S_{n}$ where $T_{n}=X_{1}+\cdots+X_{n}$ and $S_{n}=Y_{1}+\cdots+Y_{n}$. Then,

$$
\begin{aligned}
q_{2 n}\left(r_{n}\right)= & \frac{\sqrt{n} \psi_{2}^{\prime}\left(-r_{n} \tau_{n}\right)}{\left[2 \pi\left[\psi_{1}^{\prime \prime}\left(\tau_{n}\right)+r_{n}^{2} \psi_{2}^{\prime \prime}\left(-r_{n} \tau_{n}\right)\right]\right]^{1 / 2}} \\
& \times \exp \left(n\left[\psi_{1}\left(\tau_{n}\right)+\psi_{2}\left(-r_{n} \tau_{n}\right)\right]\right)[1+O(1 / n)]
\end{aligned}
$$




\subsection{Large deviation local limit theorems for ratio statistics of lattice random vari- ables}

In this section we obtain similar local limit theorems for ratio statistic $R_{n}=T_{n} / S_{n}$ when

(i) $T_{n}$ and $S_{n}$ both are lattice random variables and

(ii) when $T_{n}$ is lattice and $S_{n}$ is non-lattice.

First, we consider case (i) where both $T_{n}$ and $S_{n}$ are lattice random variables. We continue to use the same notations introduced in Section 2.3.

Theorem 2.4.1 Let $\left\{T_{n}, n \geq 1\right\}$ be a sequence of lattice random variables with distribution functions $F_{1 n}$. Let $T_{n}$ take values in the set $S_{1}=\left\{a_{n}+k h_{n}: a_{n}\right.$ and $h_{n}$ are real numbers and $k$ is an integer $\}$. We assume that the two sequences are independent. Let $\left\{S_{n}>0, n \geq 1\right\}$ be a sequence of positive lattice random variables taking values in the set $S_{2}=$ $\left\{a_{n}^{\prime}+k h_{n}^{\prime}: a_{n}^{\prime}\right.$ and $h_{n}^{\prime}$ are such that $\left.a_{n}^{\prime}+k h_{n}^{\prime}>0\right\}$. Let $r_{n}$ be an appropriate sequence of real numbers such that there exist $\tau_{n} \in J_{1}$ such that

$$
\psi_{1 n}^{\prime}\left(\tau_{n}\right)-r_{n} \psi_{2 n}^{\prime}\left(-r_{n} \tau_{n}\right)=0
$$

for $r_{n} \tau_{n} \in J_{2}, n \geq 1$. Let $\alpha_{1}, \alpha_{2}$ be positive real numbers such that

$$
\psi_{1 n}^{\prime \prime}(\tau)>\alpha_{1} \text { and } \psi_{2 n}^{\prime}\left(\tau^{\prime}\right)>\alpha_{2}
$$


for $\tau \in J_{1}$ and $\tau^{\prime} \in J_{2}$. Assume that these two sequences satisfy the following conditions: (D1) There exist $\beta_{1}<\infty$ and $\beta_{2}<\infty$ such that

$$
\left|\psi_{j n}(z)\right|<\beta_{j} \text { for } z \text { with }|z|<A_{j} \text { for } j=1,2 \text { and } n \geq 1
$$

(D2) Given $\delta>0$, there exists $0<\eta<1$ such that

$$
\limsup \sup _{\delta<|t| \leq \pi /\left|h_{n}\right|}\left|\frac{\phi_{1 n}\left(\tau_{n}+i t\right)}{\phi_{1 n}\left(\tau_{n}\right)}\right|^{1 / a_{n}}<\eta .
$$

(DS) There exist $p, l>0$ such that

$$
\sup _{\tau \in I} \int_{-\pi /\left|h_{n}\right|}^{\pi /\left|h_{n}\right|}\left|\frac{\phi_{1 n}(\tau+i t)}{\phi_{1 n}(\tau)}\right|^{l / a_{n}} d t=O\left(a_{n}^{p}\right) .
$$

Let $P_{n}(r)=P\left(T_{n}=r S_{n}\right)$. Then

$$
\begin{aligned}
\frac{\sqrt{a_{n}}}{\left|h_{n}\right|} P_{n}\left(r_{n}\right)= & {\left[\frac{1}{2 \pi\left\{\psi_{1 n}^{\prime \prime}\left(\tau_{n}\right)+r_{n}^{2} \psi_{2 n}^{\prime \prime}\left(-r_{n} \tau_{n}\right)\right\}}\right]^{1 / 2} } \\
& \times \exp \left(a_{n}\left\{\psi_{1 n}\left(\tau_{n}\right)+\psi_{2 n}\left(-r_{n} \tau_{n}\right)\right\}\right)\left[1+O\left(1 / a_{n}\right)\right] .
\end{aligned}
$$

Proof. Consider

$$
\begin{aligned}
P_{n}(r) & =P\left(T_{n}=r S_{n}\right) \\
& =\sum_{y \in S_{2}} P\left(T_{n}=r y\right) P\left(S_{n}=y\right) .
\end{aligned}
$$

Using the conjugate density technique and condition (D4), we get that

$$
P\left(T_{n}=r y\right)=\frac{\left|h_{n}\right|}{2 \pi} \int_{-\pi /\left|h_{n}\right|}^{\pi /\left|h_{n}\right|} \phi_{1 n}(\tau+i t) \exp (-(\tau+i t) r y) d t .
$$


Substituting this in (2.134), we have,

$$
\begin{aligned}
P_{n}(r) & =\sum_{y \in S_{2}} P\left(S_{n}=y\right)\left[\frac{\left|h_{n}\right|}{2 \pi} \int_{-\pi /\left|h_{n}\right|}^{\pi /\left|h_{n}\right|} \phi_{1 n}(\tau+i t) \exp (-(\tau+i t) r y) d t\right] \\
& =\frac{\left|h_{n}\right|}{2 \pi} \int_{-\pi /\left|h_{n}\right|}^{\pi /\left|h_{n}\right|} \phi_{1 n}(\tau+i t) \sum_{y \in S_{2}} P\left(S_{n}=y\right) \exp (-(\tau+i t) r y) d t \\
& =\frac{\left|h_{n}\right|}{2 \pi} \int_{-\pi /\left|h_{n}\right|}^{\pi /\left|h_{n}\right|} \phi_{1 n}(\tau+i t) \phi_{2 n}(-r(\tau+i t)) d t \\
& =\frac{\left|h_{n}\right|}{2 \pi} \int_{-\pi /\left|h_{n}\right|}^{\pi /\left|h_{n}\right|} \exp \left(a_{n}\left[\psi_{1 n}(\tau+i t)+\psi_{2 n}(-r(\tau+i t))\right]\right) d t . \quad(2.137)
\end{aligned}
$$

Replacing $r$ by $r_{n}$ and $\tau$ by $\tau_{n}$ we can rewrite $P_{n}\left(r_{n}\right)$ as follows:

$$
\begin{aligned}
\frac{\sqrt{a_{n}}}{\left|h_{n}\right|} P_{n}\left(r_{n}\right) & =\frac{\sqrt{a_{n}}}{2 \pi} \int_{-\pi /\left|h_{n}\right|}^{\pi /\left|h_{n}\right|} \exp \left(a_{n}\left[\psi_{1 n}\left(\tau_{n}+i t\right)+\psi_{2 n}\left(-r_{n}\left(\tau_{n}+i t\right)\right)\right]\right) d t \\
& =\left[\frac{1}{2 \pi\left[\psi_{1 n}^{\prime \prime}\left(\tau_{n}\right)+r_{n}^{2} \psi_{2 n}^{\prime \prime}\left(-r_{n} \tau_{n}\right)\right]}\right]^{1 / 2} \exp \left(a_{n}\left[\psi_{1 n}\left(\tau_{n}\right)+\psi_{2 n}\left(-r_{n} \tau_{n}\right)\right]\right) J_{n}
\end{aligned}
$$

where

$$
\begin{aligned}
J_{n}= & {\left[\frac{a_{n}\left[\psi_{1 n}^{\prime \prime}\left(\tau_{n}\right)+r_{n}^{2} \psi_{2 n}^{\prime \prime}\left(-r_{n} \tau_{n}\right)\right]}{2 \pi}\right]^{1 / 2} \int_{-\pi /\left|h_{n}\right|}^{\pi /\left|h_{n}\right|} \exp \left(a _ { n } \left[\psi_{1 n}\left(\tau_{n}+i t\right)\right.\right.} \\
& \left.\left.-\psi_{1 n}\left(\tau_{n}\right)+\psi_{2 n}\left(-r_{n}\left(\tau_{n}+i t\right)\right)-\psi_{2 n}\left(-r_{n} \tau_{n}\right)\right]\right) d t
\end{aligned}
$$

We can easily verify that $J_{n}$ is equal to $1+O\left(1 / a_{n}\right)$.

Thus,

$$
\begin{aligned}
\frac{\sqrt{a_{n}}}{\left|h_{n}\right|} P_{n}\left(r_{n}\right)= & {\left[\frac{1}{2 \pi\left[\psi_{1 n}^{\prime \prime}\left(\tau_{n}\right)+r_{n}^{2} \psi_{2 n}^{\prime \prime}\left(-r_{n} \tau_{n}\right)\right]}\right]^{1 / 2} } \\
& \times \exp \left(a_{n}\left[\psi_{1 n}\left(\tau_{n}\right)+\psi_{2 n}\left(-r_{n} \tau_{n}\right)\right]\right)\left[1+O\left(1 / a_{n}\right)\right]
\end{aligned}
$$


For case (ii) we have the following.

Theorem 2.4.2. Let $\left\{T_{n}, n \geq 1\right\}$ be a sequence of lattice random variables with distribution functions $F_{1 n}$ and let $\left\{S_{n}>0, n \geq 1\right\}$ be a sequence of non-lattice random variables with distribution functions $F_{2 n}$. Let $T_{n}$ take values in the set $S=\left\{a_{n}+k h_{n}: a_{n}\right.$ and $h_{n}$ are such that $\left.a_{n}+k h_{n}>0\right\}$. Let $r_{n}$ be a bounded sequence of real numbers satisfying (2.138). Suppose that Conditions (D1), (D2) and (DS) hold. If $g_{2 n}$ is the p.d.f. of $T_{n} / S_{n}$, then

$$
\begin{aligned}
\frac{\sqrt{a_{n}}}{\left|h_{n}\right|} g_{2 n}\left(r_{n}\right)= & {\left[\frac{1}{2 \pi\left[\psi_{1 n}^{\prime \prime}\left(\tau_{n}\right)+r_{n}^{2} \psi_{\cdot_{2}}^{\prime \prime}\left(-r_{n} \tau_{n}\right)\right]}\right]^{1 / 2} } \\
& \times \exp \left(a_{n}\left[\psi_{1 n}\left(\tau_{n}\right)+\psi_{2 n}\left(-\tau_{n} \tau_{n}\right)\right]\right)\left[1+O\left(1 / a_{n}\right)\right] .
\end{aligned}
$$

Proof of this theorem runs parallel to the proof of the preceding theorems and hence is omitted.

Theorems 2.4.3 and 2.4.4 stated below consider cases (i) and (ii) for i.i.d. random variables.

Theorem 2.4.3. Let $\left\{X_{n}, n \geq 1\right\}$ be a sequence of independent lattice valued random variables with common distribution function $F_{1}$ and $\left\{Y_{n}>\right.$ $0, n \geq 1\}$ be another sequence of independent and identically distributed lattice valued random variables with distribution $F_{2}$. Let the two sequences $\left\{X_{n}, n \geq 1\right\}$ and $\left\{Y_{n}, n \geq 1\right\}$ be independent of each other. Let $\phi_{1}$ and $\phi_{2}$ denote the charactereistic functions of $X_{1}$ and $Y_{1}$ respectively. Let $J_{i}=$ 
$\left(-b_{i}, b_{i}\right)$, where $0<b_{i}<c_{i}$ for some $c_{i}, i=1,2$. Let $\left\{r_{n}\right\}$ be a proper sequence of real numbers such that there exist $\tau_{n} \in J_{1}, \alpha_{1}>0$ and

$$
\psi_{1}^{\prime}\left(\tau_{n}\right)-r_{n} \psi_{2}^{\prime}\left(-r_{n} \tau_{n}\right)=0
$$

and

$$
\psi_{1}^{\prime \prime}(\tau)>\alpha_{1}
$$

Assume that $\left\{X_{n}, n \geq 1\right\}$ and $\left\{Y_{n}, n \geq 1\right\}$ satisfy the following conditions:

(E1). There exist $\beta_{i}<\infty$ such that

$$
\left|\psi_{i}(z)\right|<\beta_{i} \text { for all } z \in J_{i} \text { for } i=1,2 \text {. }
$$

(E2). There exists $B<\infty$ such that

$$
\sup _{\tau \in J_{1}} \int_{-\pi /|h|}^{\pi /|h|}\left|\frac{\phi_{1}(\tau+i t)}{\phi_{1}(\tau)}\right| d t<B
$$

Let $T_{n}=X_{1}+\cdots+X_{n}$ and $S_{n}=Y_{1}+\cdots+Y_{n}$. Then

$$
\begin{aligned}
\frac{\sqrt{n}}{|h|} P\left(T_{n}=r_{n} S_{n}\right)= & {\left[\frac{1}{2 \pi\left\{\psi_{1}^{\prime \prime}\left(\tau_{n}\right)+r_{n}^{2} \psi_{2}^{\prime \prime}\left(-r_{n} \tau_{n}\right)\right\}}\right]^{1 / 2} } \\
& \exp \left(n\left[\psi_{1}\left(\tau_{n}\right)+\psi_{2}\left(-r_{n} \tau_{n}\right)\right]\right)[1+O(1 / n)] .
\end{aligned}
$$

Theorem 2.4.4 Let $\left\{X_{n}, n \geq 1\right\}$ be a sequence of independent and identically distributed lattice valued random variables with distribution function $F_{1}$. Let $\left\{Y_{n}>0, n \geq 1\right\}$ be another sequence of non-lattice and independent random variables with common distribution function $F_{2}$. Assume that 
the two sequences satisfy the conditions of Theorem 2.4.3. The probability density function $g_{2 n}$ of $T_{n} / S_{n}$ is given by

$$
\begin{aligned}
\frac{\sqrt{n}}{|h|} g_{2 n}\left(r_{n}\right)= & {\left[\frac{1}{2 \pi\left\{\psi_{1}^{\prime \prime}\left(\tau_{n}\right)+r_{n}^{2} \psi_{2}^{\prime \prime}\left(-r_{n} \tau_{n}\right)\right\}}\right]^{1 / 2} } \\
& \times \exp \left(n\left[\psi_{1}\left(\tau_{n}\right)+\psi_{2}\left(-r_{n} \tau_{n}\right)\right]\right)[1+O(1 / n)] \cdot(2.147)
\end{aligned}
$$




\subsection{Applications}

In this section we present a number of examples to illustrate large deviation local limit theorems for the ratio statistic $R_{n}=T_{n} / S_{n}$ obtained in Sections 2.3 and 2.4. Our examples cover all the four combinations of non- lattice and lattice cases for $T_{n}$ and $S_{n}$. One should note that in all these examples the exact density does not have a closed form, however our theorems provide a simple asymptotic expressions. These examples clearly demonstrate the wide applicability of our theorems. To simplify matters in all the examples we choose $T_{n}$ and $S_{n}$ to be the sum of $n$ i.i.d. random variables. The first Example 2.5.1 considers the case of non-lattice for both $T_{n}$ and $S_{n}$. In this example we choose $T_{n}$ to be the sum of $n$ i.i.d. $N(0,1)$ random variables and $S_{n}$ to be the sum of $n$ i.i.d. $\chi^{2}$ random variables with one degree of freedom. We verify easily all the conditions of Theorem 2.3.13. Thus the conclusion (2.125) of Theorem 2.3.13 yields a simple expression for the density of the random variable which is the ratio of $N(o, n)$ and $\chi^{2}$ with $n$ degrees of freedom. The second Example 2.5.2 considers the case of non-lattice for $T_{n}$ and lattice for $S_{n}$. Here we choose $T_{n}$ to be the same as in Example 2.5.1 but $S_{n}$ is chosen to be the sum of $n$ i.i.d. Poisson random variables with mean equal to 1. Proceeding as in Example 2.5.1 we can easily verify that all the conditions (B1), (B2) and (B3) of Theorem 2.3.14 are satisfied. Thus we obtain in (2.153) an asymptotic expression for the density of the ratio statistic $N(0, n)$ over Poisson $(n)$ at a suitable point $r_{n}$. In the third Example 2.5.3 we consider the case of lattice random variables $T_{n}$ and $S_{n}$. We chose both $T_{n}$ and $S_{n}$ to be the sum of $n$ independent Poisson 
random variables each with means $\lambda_{1}$ and $\lambda_{2}$ respectively. As mentioned earlier the probability mass function of $T_{n} / S_{n}$ can only be written as an infinite series and does not have a closed form. However, we easily verify all the conditions of Theorem 2.4.3 and thus we obtain a simple closed form asymptotic expression for the probability mass function of $T_{n} / S_{n}$.

Finally we obtain an approximation for the density function of consider the $F$ statistic with $n$ degrees of freedom for both the numerator and denominator. We assume that $n$ is large. In this case we show that our approximation for the density agrees with the exact expression except for the normalizing constant. However, we show that the ratio of two constants converges to one as $n \rightarrow \infty$, directly instead of appealing to Theorem 2.3.13.

Example 2.5.1. Let $\left\{X_{n}, n \geq 1\right\}$ be a sequence of independent normal random variables with mean 0 and variance 1 . Let $\left\{Y_{n}, n \geq 1\right\}$ be a sequence of independent random variables with common distribution function $\chi^{2}$ with one degree of freedom. The c.f. of $X_{1}$ is given by $\phi_{1}(z)=e^{z^{2} / 2}$ and that of $Y_{1}$ is given by $\phi_{2}(z)=(1-2 z)^{-1 / 2}$ for $|z|<1 / 2$. Let $r_{n}$ be a bounded sequence of real numbers such that $0<\underline{r}<r_{n}<\bar{\tau}$. Let

$$
\tau_{n}=\frac{-1+\sqrt{1+8 r_{n}^{2}}}{4 r_{n}} .
$$

Let $b_{1}=\left(-1+\sqrt{1+8 \bar{r}^{2}}\right) / 2 \underline{r}$ and $c_{1}=2 b_{1}$. Further, let $\left(-1+\sqrt{1+8 \bar{r}^{2}}\right) / 4<b_{2}<\min \left\{\left(-1+\sqrt{1+8 \bar{r}^{2}}\right) / 2,1 / 2\right\}$ and $c_{2}=1 / 2$.

We now verify all the conditions of Theorem 2.3.13.

Condition (B1). Let $\beta_{1}=c_{1}^{2}$ and $\beta_{2}=\sqrt{[\log 4]^{2}+4 \pi^{2}}$. Then

$$
\left|\psi_{1}(z)\right|=\frac{|z|^{2}}{2}<\beta_{1}, \text { for }|z|<c_{1}
$$


and

$$
\left|\psi_{2}(z)\right|=|\log (1-2 z)|<\beta_{2}, \text { for }|z|<1 / 2
$$

Condition (B2). Let $\delta>0$. Then

$$
\begin{aligned}
\sup _{|t|>\delta}\left|\psi_{2}^{\prime}\left(-r_{n}\left(\tau_{n}+i t\right)\right)\right| & =\sup _{|t|>\delta}\left|\frac{1}{\left[1-2\left(-r_{n}\left(\tau_{n}+i t\right)\right)\right]}\right| \\
& =\sup _{|t|>\delta} \frac{1}{\sqrt{\left(1+2 r_{n} \tau_{n}\right)^{2}+4 r_{n}^{2} t^{2}}} \\
& <q \\
& <\infty
\end{aligned}
$$

where $q=\sup _{n} q_{n}$ and $q_{n}=1 / \sqrt{\left(1+2 \underline{r} \tau_{n}\right)^{2}+4 \underline{r}^{2} \delta^{2}}$.

Condition (B3). This condition is trivially satisfied.

Thus we have verified all the conditions of Theorem 2.3.13. An asymptotic expression for the density function of $R_{n}=T_{n} / S_{n}$ where $T_{n}=X_{1}+$ $\cdots+X_{n}$ and $S_{n}=Y_{1}+\cdots+Y_{n}$ is given by

$$
q_{1 n}\left(r_{n} \tau_{n}\right)=\sqrt{\frac{n}{2 \pi}} \frac{\left(1+2 r_{n} \tau_{n}\right)^{-n / 2}}{\left[2+\left(1+2 r_{n} \tau_{n}\right)^{2}\right]^{1 / 2}} \exp \left(\frac{n \tau_{n}^{2}}{2}\right)[1+O(1 / n)] .
$$

Example 2.5.2. Let $\left\{X_{n}, n \geq 1\right\}$ be a sequence of independent random variables with common distribution function $N(0,1)$. Let $\left\{Y_{n}, n \geq 1\right\}$ be a sequence of Poisson random variables with parameter $\lambda=1$. Let $\phi_{1}(z)=$ $e^{z^{2} / 2}$ and $\phi_{2}(z)=e^{\left(e^{z}-1\right)}$. We choose $c_{i}>0$ such that $\phi_{i}$ is non-vanishing and analytic in $\Omega_{i}=\left\{z \in C:|z|<c_{i}\right\}$, for $i=1,2$. Let $J_{i}=\left(-b_{i}, b_{i}\right)$, 
$0<b_{i}<c_{i}$ for $i=1,2$. We choose $b_{i}^{\prime} s$ in such a fashion so that for a bounded sequence $r_{n}$ we can always find $\tau_{n} \in J_{1}$ satisfying

$$
\tau_{n} e^{r_{n} \tau_{n}}=r_{n}
$$

and $r_{n} \tau_{n} \in J_{2}$. In this example we can easily verify Conditions (B1), (B2) and (B3) of Theorem 2.3.14. Thus the density function $g_{2 n}$ of $T_{n} / S_{n}$ at the point $r_{n}$ is given by

$$
q_{2 n}\left(r_{n}\right)=\frac{\sqrt{n} e^{-r_{n} \tau_{n}}}{\left[2 \pi\left[1+r_{n}^{2} e^{-\tau_{n} \tau_{n}}\right]\right]^{1 / 2}} \exp \left(n\left[\frac{2 e^{\tau_{n}}+\tau_{n}^{2}-2}{2}\right]\right)[1+O(1 / n)]
$$

Example 2.5.3. Let $\left\{X_{n}, n \geq 1\right\}$ be a sequence of Poisson random variables with parameter $\lambda_{1}$. Let $\left\{Y_{n}, n \geq 1\right\}$ be another sequence of Poisson random variables with parameter $\lambda_{2}$. Let $T_{n}=X_{1}+\cdots+X_{n}$ and $S_{n}=Y_{1}+\cdots+Y_{n}$. Then $\phi_{i}(z)=e^{\lambda_{i}\left(e^{z}-1\right)}$ and $\psi_{i}(z)=\lambda_{i}\left(e^{z}-1\right)$ for $i=1,2$. Note that $\phi_{1}(z)$ and $\phi_{2}(z)$ are analytic in all the complex plane C. Let $J_{i}=\left(-b_{i}, b_{i}\right)$ for $0<b_{i}<c_{i}$ for $i=1,2$. Let us choose a bounded sequence of real numbers $\left\{r_{n}\right\}$ such that $0<\underline{r}<r_{n}<\bar{r}$ and there exist $\tau_{n}^{\prime} s$ satisfying

$$
\tau_{n}=\frac{\log r_{n}+\log \lambda_{2}-\log \lambda_{1}}{\left(r_{n}+1\right)}
$$

Let $b_{1}=2 \log \bar{\tau}+\log \lambda_{2}-\log \lambda_{1} /(\underline{r}+1)$ and $c_{1}=2 b_{1}$. Let $b_{2}=\log \bar{\tau}+$ $\log \lambda_{2}-\log \lambda_{1}$ and $c_{2}=2 b_{2}$. With $\alpha_{1}=\lambda_{1}^{(\bar{r}+1) / 2} / 2|\underline{r}| \lambda_{2}{ }^{(\underline{r}+1)}$ we have $\psi_{1}^{\prime \prime}(\tau)=$ $\lambda_{1} e^{\tau}>\alpha_{1}$. We now proceed to verify all the conditions of Theorem 2.4.3. Condition (E1). Let $\beta_{i}=2 \lambda_{i}\left(e^{c}+1\right)^{2}$ for $i=1,2$. For this $\beta_{i}$ it is easy to check that $|z|<c_{i}$ implies $\left|\psi_{i}(z)\right|<\beta_{i}$ for $i=1,2$.

Condition (E2) is trivially satisfied. 
Thus from Theorem 2.4.3 we can write an asymptotic expression for $q_{3 n}\left(r_{n}\right)=P\left(T_{n}=r S_{n}\right)$ which is given by

$$
\begin{aligned}
q_{3 n}\left(r_{n}\right)= & \frac{1}{\left[2 \pi n\left[\lambda_{1} e^{\tau_{n}}+\lambda_{2} r_{n}^{2} e^{-\tau_{n} \tau_{n}}\right]\right]^{1 / 2}} \\
& \times \exp n\left[\lambda_{1}\left(e^{\tau_{n}}-1\right)+\lambda_{2}\left(e^{-\tau \tau_{n}}-1\right)\right][1+O(1 / n)] .
\end{aligned}
$$

Let $F_{n, n}$ denote the $F$ statistic with $n, n$ degrees of freedom. In the next example we compare, for large values of $\mathrm{n}$, exact density of $F_{n, n}$ statistic with the asymptotic expression of the density given by (2.60).

Example 2.5.4. Let $T_{n}$ and $S_{n}$ be the sums of $n$ i.i.d. $\chi^{2}$ random variables with one degree of freedom. The c.f. of $T_{n}$ is given by $\phi_{1}(z)=(1-2 z)^{-n / 2}$ and that of $S_{n}$ is given by $\phi_{2}(z)=(1-2 z)^{-n / 2}$. Both $\phi_{1}(z)$ and $\phi_{2}(z)$ are analytic and non-vanishing in $\Omega=\{z \in C:|z|<1 / 2\}$. Let $J_{i}=\left(-b_{i}, b_{i}\right)$ for $0<b_{i}<1 / 2$. We now briefly verify conditions of Theorem 2.3.13.

Condition (A1). Let $\beta_{i}=\sqrt{[\log 4]^{2}+4 \pi^{2}}$ for $i=1,2$. Then for $|z|<1 / 2$ it follows that, $\left|\psi_{i}(z)\right|<\beta_{i}$ for $i=1,2$.

Condition (A2). This is verified as in Example 2.5.1.

Condition(A3). Consider

$$
\begin{aligned}
\left|\frac{\phi_{1}(\tau+i t)}{\phi_{1}(\tau)}\right| & =\left|\frac{1-2 \tau}{(1-2(\tau+i t))}\right|^{1 / 2} \\
& =\frac{(1-2 \tau)^{1 / 2}}{\left[(1-2 \tau)^{2}+4 t^{2}\right]^{1 / 4}}
\end{aligned}
$$


Thus

$$
\begin{aligned}
\sup _{\tau \in J_{1}} \int_{-\infty}^{\infty}\left|\frac{\phi_{1}(\tau+i t)}{\phi_{1}(\tau)}\right| d t & =\sup _{\tau \in J_{1}} \int_{-\infty}^{\infty} \frac{(1-2 \tau)^{1 / 2}}{\left[(1-2 \tau)^{2}+4 t^{2}\right]^{1 / 4}} d t \\
& =\sup _{\tau \in J_{2}}(1-2 \tau)^{1 / 2} \int_{-\infty}^{\infty} \frac{1}{\left[(1-2 \tau)^{2}+4 t^{2}\right]^{1 / 4}} d t \\
& \leq \sqrt{2} \pi .
\end{aligned}
$$

Thus we have verified all the conditions of Theorem 2.3.13 and we now apply the same theorem (see (2.60)). On simplification of the numerator and denominator in the expression (2.60) we get

$$
q_{1 n}\left(r_{n}\right)=\frac{n^{1 / 2} 2^{n-1 / 2}}{\sqrt{2 \pi}} \frac{r_{n}^{n / 2-1}}{\left(r_{n}+1\right)^{n}}
$$

where $\left\{r_{n}\right\}$ is an appropriate sequence of real numbers. The exact density of the ratio $T_{n} / S_{n}$ at point $r$ is given by

$$
f\left(r_{n} \mid n, n\right)=\frac{(n-1) !}{\left(\frac{n}{2}-1\right) !\left(\frac{n}{2}-1\right) !} \frac{r_{n}^{n / 2-1}}{\left(r_{n}+1\right)^{n}} .
$$

On comparing (2.159) and (2.160) we see that both the expressions agree except for a constant. We can use Stirling's formula and show that the ratio of the two constants occurring in (2.159) and (2.160) tends to 1 as $n \rightarrow \infty$. 


\section{Chapter 3}

\section{Large deviation local limit}

\section{theorems for random vectors}

In this chapter we shall extend the local limit theorems for ratio statistics of random variables in Chapter 2 to random vectors. We begin with a few definitions and notations.

\subsection{Definitions and Preliminaries.}

We denote the $k$-dimensional complex plane by $C^{k}$. The points in $C^{k}$ are denoted by $z=\left(z_{1}, z_{2}, \cdots, z_{k}\right)$ where $z_{i} \in C, i=1,2, \cdots, k$. If $\alpha=\left(\alpha_{1}, \alpha_{2}, \cdots, \alpha_{k}\right)$ is a $k$-dimensional vector with nonnegative integer components, we shall use the following abbrevations:

$$
\begin{aligned}
& z^{\alpha}=z_{1}^{\alpha_{1}} z_{2}^{\alpha_{2}} \cdots z_{k}^{\alpha_{k}} \\
& d z=d z_{1} d z_{2} \cdots d z_{k}
\end{aligned}
$$




$$
<z, \xi>=z_{1} \xi_{1}+z_{2} \xi_{2}+\cdots+z_{k} \xi_{k}
$$

for all $z, \xi \in C^{k}$.

$$
|\alpha|=\left|\alpha_{1}\right|+\left|\alpha_{2}\right|+\cdots+\left|\alpha_{k}\right|
$$

When $f$ is a complex valued function defined on $C^{k}$ and $i, j, l$ are positive integers we write

$$
\begin{gathered}
D_{i j} f(z)=\frac{D^{2} f(z)}{d z_{i} d z_{j}}, \\
D_{i j l} f(z)=\frac{D^{3} f(z)}{d z_{i} d z_{j} d z_{l}},
\end{gathered}
$$

and

$$
D^{\alpha} f(z)=\frac{D^{|\alpha|} f(z)}{d z_{1}^{\alpha_{1}} d z_{2}^{\alpha_{2}} \cdots d z_{k}^{\alpha_{k}}} .
$$

Definition 3.1.1. A polydisc $s(z, r)$ of radius $r=\left(r_{1}, r_{2}, \cdots, r_{k}\right)$ around a point $z$ is defined as

$$
s(z, r)=s\left(z_{1}, r_{1}\right) \times s\left(z_{2}, r_{2}\right) \times \cdots \times s\left(z_{k}, r_{k}\right)
$$

where $s\left(z_{i}, r_{i}\right)=\left[z_{i}^{\prime} \in C:\left|z_{i}^{\prime}-z_{i}\right|<r_{i}\right]$ for $i=1,2, \cdots, k$.

Definition 3.1.2. A closed polydisc $s(z, r)$ of radius $r=\left(r_{1}, r_{2}, \cdots, r_{k}\right)$ around a point $z$ is defined as

$$
\bar{s}(z, r)=\bar{s}\left(z_{1}, r_{1}\right) \times \bar{s}\left(z_{2}, r_{2}\right) \times \cdots \times \bar{s}\left(z_{k}, r_{k}\right)
$$

where $\bar{s}\left(z_{i}, r_{i}\right)=\left[z_{i}^{\prime} \in \mathcal{C}:\left|z_{i}^{\prime}-z_{i}\right| \leq r_{i}\right]$ for $i=1,2, \cdots, k$. 
Definition 3.1.3. A complex valued function $f$ is said to be holomorphic at a point $z_{0} \in C^{k}$ if in some neighbourhood of $z_{0}$, it is the sum of an absolutely convergent power series

$$
f(z)=\sum_{|\alpha| \geq 0} a_{\alpha}\left(z-z_{0}\right)^{\alpha}
$$

Let $\left(z+r e^{i \theta}\right)=\left(z_{1}+r_{1} e^{i \theta_{1}}, \cdots, z_{k}+r_{k} e^{i \theta_{k}}\right)$ for $r=\left(r_{1}, r_{2}, \cdots, r_{k}\right)$ and $\theta=\left(\theta_{1}, \theta_{2}, \cdots, \theta_{k}\right)$. The following theorem can be found in Vladimirov (1966) pp. 30-31.

Theorem 3.1.4 (Cauchy). Suppose that a function $f(z)$ is holomorphic and that it is bounded in the closed polydisc $\bar{s}\left(z_{0}, r\right)$ then the coefficient $a_{\alpha}$ in the expansion of $f$ is given by

$$
a_{\alpha}=\frac{1}{(2 \pi)^{k}} \int_{0}^{2 \pi} \cdots \int_{0}^{2 \pi} \frac{f\left(z_{0}+r e^{i \theta}\right)}{r^{\alpha}} \exp (-i<\theta, \alpha>) d \theta .
$$

Consequently,

$$
\left|a_{\alpha}\right| \leq \frac{1}{r^{\alpha}} \max _{z \in \bar{s}\left(z_{0}, r\right)}|f(z)|
$$

\subsection{Local limit theorems for random vectors}

Let $\left\{T_{n}, n \geq 1\right\}$ be a sequence of non-lattice random vectors in $R^{k}$ and let $\left\{S_{n}>0, n \geq 1\right\}$ be a sequence of non-lattice random variables. Let us assume that $S_{n}$ is independent of $T_{n}$ for $n \geq 1$. Let the moment generating function $\phi_{1 n}(z)=E\left(\exp <z, T_{n}>\right)$ of $\mathcal{T}_{n}$ be holomorphic in $\Omega_{1}^{k}$, where $\Omega_{1}=\left\{x+i y: x \in I=\left(-c_{1}, c_{1}\right)\right.$ and $\left.y \in R\right\}$ for some $c_{1}>0$. Let $\phi_{2 n}(z)=E\left(\exp \left(z S_{n}\right)\right)$ be analytic in $\Omega_{2}=\left\{z \in \mathcal{C}:|z|<c_{2}\right\}$ for some 
$c_{2}>0$. Let $C$ denote the set of all complex numbers. Let $J_{1}=\left(-b_{1}, b_{1}\right)$, $0<b_{1}<c_{1}$ and $J_{2}=\left(-b_{2}, b_{2}\right), 0<b_{2}<c_{2}$. Let $\left\{a_{n}\right\}$ be a sequence of real numbers such that $a_{n} \rightarrow \infty$.

Let

$$
\begin{aligned}
& \psi_{1 n}(z)=\frac{1}{a_{n}} \log \phi_{1 n}(z), \text { for } z \in \Omega_{1}^{k} . \\
& \psi_{2 n}(z)=\frac{1}{a_{n}} \log \phi_{2 n}(z), \text { for } z \in \Omega_{2} .
\end{aligned}
$$

Let $\nabla \psi_{1 n}(z)=\left(D_{1} \psi_{1 n}(z), D_{2} \psi_{1 n}(z), \cdots, D_{k} \psi_{1 n}(z)\right)$ be the vector of the first order partial derivatives and $\nabla^{2} \psi_{1 n}(z)$ denote the matrix of second order partial derivatives, that is,

$$
\nabla^{2} \psi_{1 n}(z)=\left(D_{i j} \psi_{1 n}(z)\right)
$$

The determinant of the matrix $\nabla^{2} \psi_{1 n}(z)$ is denoted by $\left|\nabla^{2} \psi_{1 n}(z)\right|$.

Let $d_{n}=\nabla^{2}\left(\psi_{1 n}(\tau)+\psi_{2 n}(-<r, \tau>)\right)$ be positive definite. Further, let the eigen values of $d_{n}$ be bounded below by $\alpha_{1}>0$, for all $\tau \in J_{1}^{k}$ and $n \geq 1$. Let $\left\{r_{n}\right\}$ be a sequence of vectors in $R^{k}$ such that $\left|r_{j n}\right|<\overline{r_{j}}<\infty$ for $j=1, \cdots, k$ and $n \geq 1$. For this sequence $r_{n}$ let there exist a sequence $\left\{\tau_{n}\right\}$ in $J_{1}^{k}$ such that $\left\langle r_{n}, \tau_{n}>\in J_{2}\right.$ and

$$
\nabla\left(\psi_{1 n}\left(\tau_{n}\right)+\psi_{2 n}\left(-<<\tau_{n}, \tau_{n}>\right)\right)=0
$$

and

$$
\psi_{2 n}^{\prime}\left(-<r_{n}, \tau_{n}>\right)>\alpha_{2}
$$

for some positive constant $\alpha_{2}$ and for all $n \geq 1$.

We now state the main theorem of this section. 
Theorem 3.2.1. Let $\left\{T_{n}, n \geq 1\right\}$ be a sequence of random vectors in $R^{k}$. Let $\left\{S_{n}, n \geq 1\right\}$ be a sequence of random variables which is independent of $T_{n}$. Assume that $T_{n}$ and $S_{n}$ satisfy the following conditions:

(F1) There exist $\beta_{1}<\infty$ and $\beta_{2}<\infty$ such that

$$
\left|\psi_{1 n}(z)\right|<\beta_{1} \text { for } z \in \Omega_{1}^{k}
$$

and

$$
\left|\psi_{2 n}(z)\right|<\beta_{2} \text { for } z \in \Omega_{2} \text { for all } n \geq 1 \text {. }
$$

(F2) Given $\delta>0$ there exist $0<\eta<1, q>0$ such that

$$
\underset{n}{\limsup } \sup _{|t|>\delta}\left|\frac{\phi_{1 n}\left(\tau_{n}+i t\right)}{\phi_{1 n}\left(\tau_{n}\right)}\right|^{1 / a_{n}}<\eta
$$

and

$$
\sup _{|t|>\delta}\left|\psi_{2 n}^{\prime}\left(-<r_{n}, \tau_{n}+i t>\right)\right|=O\left(a_{n}^{q}\right)
$$

(FS) There exist $p, l>0$ such that

$$
\sup _{\tau \in J_{1}^{k}} \int_{-\infty}^{\infty}\left|\frac{\phi_{1 n}(\tau+i t)}{\phi_{1 n}(\tau)}\right|^{l / a_{n}} d t=O\left(a_{n}^{p}\right)
$$

Then, an asymptotic expansion for the density function $\bar{g}_{n}$ of $T_{n} / S_{n}$ at the point $r_{n}$ is given by,

$$
\begin{aligned}
\bar{g}_{n}\left(r_{n}\right)= & \frac{a_{n}^{k / 2}\left(\psi_{2 n}^{\prime}\left(-<r_{n}, \tau_{n}>\right)\right)^{k}}{(2 \pi)^{k / 2}\left|d_{n}\right|^{1 / 2}} \\
& \times \exp \left(a_{n}\left[\psi_{1 n}\left(\tau_{n}\right)+\psi_{2 n}\left(-<r_{n}, \tau_{n}>\right)\right]\right)\left[1+O\left(1 / a_{n}\right)\right] .
\end{aligned}
$$


Remark 3.2.3. The proof of Theorem 3.2.1 is similar to that of Theorem 2.3.1. The proof has two major steps. In the first step the term $I_{n 1}$ appearing in the proof of the theorem is shown to go to zero exponentially fast and in the second step the term $I_{n 2}$ is shown to be equal to $1+O\left(1 / a_{n}\right)$. These steps are presented below as Lemmas 3.2.7 and 3.2.9 respectively. The proofs of these lemmas depend on lemmas 3.2.4, 3.2.8 which are also proved below.

Lemma 3.2.4. Let $\psi_{1 n}$ and $\psi_{2 n}$ be as defined in (\$.14) and (\$.15). Assume that Condition (F1) of Theorem 3.2.1 holds. Let $\delta_{1}$ be any real number less than $\left(c_{1}-b_{1}\right) / 2$. For $|t|<\delta_{1}$ let

$$
\begin{gathered}
R_{1 n}\left(\tau_{n}+i t\right)=\psi_{1 n}\left(\tau_{n}+i t\right)-\psi_{1 n}\left(\tau_{n}\right)-i<t, \nabla \psi_{1 n}\left(\tau_{n}\right)> \\
+\frac{1}{2} t^{\prime} \nabla^{2} \psi_{1 n}\left(\tau_{n}\right) t+i \sum_{|\alpha|=3} a_{\alpha}^{(n)} t^{|\alpha|} .
\end{gathered}
$$

Then we have the following bound:

$$
\sup _{n}\left|R_{1 n}\left(\tau_{n}+i t\right)\right| \leq \frac{2 \beta_{1} t^{4}}{\left(c_{1}-b_{1}\right)^{4}} .
$$

Proof. Since $\tau_{n} \in J_{1}^{k}$ for all $n \geq 1$ and $\psi_{1 n}$ is holomorphic in $\Omega_{1}^{k}$ we can write for $|t|<\left(c_{i}-b_{i}\right) / 2$,

$$
\begin{gathered}
\psi_{1 n}\left(\tau_{n}+i t\right)=\psi_{1 n}\left(\tau_{n}\right)+i<t, \nabla \psi_{1 n}\left(\tau_{n}\right)>-\frac{1}{2} t^{\prime} \nabla^{2} \psi_{1 n}\left(\tau_{n}\right) t \\
-i \sum_{|\alpha|=3} a_{\alpha}^{(n)} t^{|\alpha|}+\sum_{|\alpha| \geq 4} a_{\alpha}^{(n)}(i t)^{|\alpha|} .
\end{gathered}
$$


By Cauchy's theorem and Condition (F1) of Theorem 3.2.1 we get the following bound for $a_{\alpha}^{(n)}$

$$
\left|a_{\alpha}^{(n)}\right| \leq \frac{\beta_{1}}{\left(c_{1}-b_{1}\right)^{|\alpha|}} .
$$

Thus for $|t|<\left(c_{1}-b_{1}\right) / 2$ and for all $n \geq 1$,

$$
\begin{aligned}
\left|R_{1 n}\left(\tau_{n}+i t\right)\right| & =\left|\sum_{|\alpha| \geq 4} a_{\alpha}^{(n)}(i t)^{|\alpha|}\right| \\
& \leq \sum_{|\alpha| \geq 4}\left[\prod_{j=1}^{k}\left|t_{j}\right|^{\alpha_{j}}\right] \frac{\beta_{1}}{\left(c_{1}-b_{1}\right)^{|\alpha|}} \\
& \leq \frac{2|t|^{4} \beta_{1}}{\left(c_{1}-b_{1}\right)^{4}} .
\end{aligned}
$$

Remark 3.2.5. Let $R_{2 n}(\tau+i t)$ be as defined in (2.62). As shown in Lemma 2.3.7 there exists $0<\delta_{2}<\left(c_{2}-b_{2}\right) / 2$ such that

$$
\sup _{\tau \in J_{2}}\left|R_{2 n}(\tau+i t)\right| \leq \frac{2 \beta_{2} t^{4}}{\left(c_{2}-b_{2}\right)^{4}}
$$

for $|t|<\delta_{2}$ and for all $n \geq 1$.

Remark 3.2.6. Let $\sup _{n}\left|r_{n}\right|=r$. Let $\delta_{3}=\delta_{2} / r$. Let $R(z)$ be the remainder term in the expansion of $\left.\psi_{2 n}^{\prime}\left(-<\tau_{n}, z\right\rangle\right)$ as in (2.64). Proceeding as in Remark 2.3.8. we can show that for $|t|<\delta_{3}$,

$$
\left|R\left(-<r_{n}, \tau_{n}+i t>\right)\right| \leq \frac{2 \beta_{2}<r_{n}, t>^{3}}{\left(c_{2}-b_{2}\right)^{3}} .
$$

In the next lemma we show that the term $I_{n 1}$ defined in (3.29) goes to zero exponentially fast. 
Lemma 3.2.7. Assume that the Conditions (F2) and (FY) of Theorem 3.2.1 are satisfied. Let

$$
d_{n}=\nabla^{2}\left(\psi_{1 n}\left(\tau_{n}\right)+\psi_{2 n}\left(-<\tau_{n}, \tau_{n}>\right)\right)
$$

and

$$
f_{1 n}(z)=\psi_{1 n}(z)+\psi_{2 n}\left(-<r_{n}, z>\right) \text {. }
$$

Then there exists $\delta>0$ such that

$$
\begin{gathered}
I_{n 1}=\left[\frac{\left.a_{n}^{k}\left|d_{n}\right|\right]^{1 / 2}}{(2 \pi)^{k}} \int_{|t| \geq \delta} \exp \left(\left[a_{n}\left[f_{n}\left(\tau_{n}+i t\right)-f_{n}\left(\tau_{n}\right)\right]\right]\right)\right. \\
\frac{\psi_{2 n}^{\prime}\left(-<r_{n}, \tau_{n}+i t>\right)}{\psi_{2 n}^{\prime}\left(-<r_{n}, \tau_{n}>\right)} d t
\end{gathered}
$$

goes exponentially fast to zero.

Proof. Let $\delta$ be such that $\delta<\min \left(\delta_{1}, \delta_{2}, \delta_{3}\right)$ where $\delta_{1}, \delta_{2}$ and $\delta_{3}$ are as in Lemma 3.2.4, Remark 3.2.5 and Remark 3.2.6 respectively. Consider

$$
\begin{aligned}
\left|I_{n 1}\right|= & \left.\mid\left[\frac{a_{n}^{k}\left|d_{n}\right|}{(2 \pi)^{k}}\right]^{1 / 2} \int_{|t| \geq \delta} \exp \left(\left[a_{n} \mid f_{n}\left(\tau_{n}+i t\right)-f_{n}\left(\tau_{n}\right)\right]\right]\right) \\
& \frac{\psi_{2 n}^{\prime}\left(-<r_{n}, \tau_{n}+i t>\right)}{\psi_{2 n}^{\prime}\left(-<r_{n}, \tau_{n}>\right)} d t \mid \\
\leq & {\left[\frac{a_{n}^{k}\left|d_{n}\right|}{(2 \pi)^{k}}\right]^{1 / 2} \int_{|t| \geq \delta}\left|\exp \left(\left[a_{n}\left[f_{n}\left(\tau_{n}+i t\right)-f_{n}\left(\tau_{n}\right)\right]\right]\right)\right| } \\
& \times\left|\frac{\psi_{2 n}^{\prime}\left(-<r_{n}, \tau_{n}+i t>\right)}{\psi_{2 n}^{\prime}\left(-<r_{n}, \tau_{n}>\right)}\right| d t .
\end{aligned}
$$

Substituting for $f_{n}\left(\tau_{n}+i t\right)$ and $f_{n}\left(\tau_{n}\right)$ (see (3.28)), we get that

$$
\left|I_{n 1}\right| \leq\left[\frac{a_{n}^{k}\left|d_{n}\right|}{(2 \pi)^{k}}\right]^{1 / 2} \int_{|t| \geq \delta}\left|\frac{\phi_{1 n}\left(\tau_{n}+i t\right)}{\phi_{1 n}\left(\tau_{n}\right)} \frac{\phi_{2 n}\left(-<r_{n}, \tau_{n}+i t>\right)}{\phi_{2 n}\left(-<r_{n}, \tau_{n}>\right)}\right|
$$




$$
\begin{aligned}
& \times\left|\frac{\psi_{2 n}^{\prime}\left(-<\tau_{n}, \tau_{n}+i t>\right)}{\psi_{2 n}^{\prime}\left(-<r_{n}, \tau_{n}>\right)}\right| d t \\
& \leq\left[\frac{a_{n}^{k}\left|d_{n}\right|}{(2 \pi)^{k}}\right]^{1 / 2} \int_{|t| \geq \delta}\left|\frac{\phi_{1 n}\left(\tau_{n}+i t\right)}{\phi_{1 n}\left(\tau_{n}\right)}\right|\left|\frac{\psi_{2 n}^{\prime}\left(-<r_{n}, \tau_{n}+i t>\right)}{\psi_{2 n}^{\prime}\left(-<r_{n}, \tau_{n}>\right)}\right| d t \\
& \leq\left[\frac{a_{n}^{k}\left|d_{n}\right|}{(2 \pi)^{k}}\right]^{1 / 2} \sup _{|t| \geq \delta}\left|\frac{\psi_{2 n}^{\prime}\left(-<r_{n}, \tau_{n}+i t>\right)}{\psi_{2 n}^{\prime}\left(-<r_{n}, \tau_{n}>\right)}\right| \\
& \times \int_{|t| \geq \delta}\left|\frac{\phi_{1 n}\left(\tau_{n}+i t\right)}{\phi_{1 n}\left(\tau_{n}\right)}\right|^{l / a_{n}}\left|\frac{\phi_{1 n}\left(\tau_{n}+i t\right)}{\phi_{1 n}\left(\tau_{n}\right)}\right|^{\left(1-l / a_{n}\right)} d t \\
& \leq\left[\frac{a_{n}^{k}\left|d_{n}\right|}{(2 \pi)^{k}}\right]^{1 / 2} \sup _{|t| \geq \delta}\left|\frac{\psi_{2 n}^{\prime}\left(-<r_{n}, \tau_{n}+i t>\right)}{\psi_{2 n}^{\prime}\left(-<r_{n}, \tau_{n}>\right)}\right| \sup _{|t| \geq \delta}\left|\frac{\phi_{1 n}\left(\tau_{n}+i t\right)}{\phi_{1 n}\left(\tau_{n}\right)}\right|^{\left(1-l / a_{n}\right)} \\
& \times \int_{|t| \geq \delta}\left|\frac{\phi_{i n}\left(\tau_{n}+i t\right)}{\phi_{1 n}\left(\tau_{n}\right)}\right|^{l / a_{n}} d t
\end{aligned}
$$

From (3.17), (3.18) and (3.19) and also noting the fact that $\psi_{2 n}^{\prime}\left(-<r_{n}, \tau_{n}>\right)>\alpha_{2}$ we get that

$$
\begin{aligned}
\left|I_{n 1}\right| & \leq\left[\frac{a_{n}^{k}\left|d_{n}\right|}{(2 \pi)^{k}}\right]^{1 / 2} \frac{1}{\alpha_{2}} O\left(a_{n}^{q}\right) \eta^{\left(1-l / a_{n}\right)} \int_{R^{k}}\left|\frac{\phi_{1 n}\left(\tau_{n}+i t\right)}{\phi_{1 n}\left(\tau_{n}\right)}\right|^{l / a_{n}} d t \\
& \leq O\left(a_{n}^{p+q+k / 2}\right) \exp \left(-\eta_{1}\left(a_{n}-l\right)\right)
\end{aligned}
$$

which goes to zero exponentially fast, since $\eta_{1}=-\log \eta>0$.

The following lemma will be used to prove Lemma 3.2.9.

Lemma 3.2.8. Let $\psi_{1 n}$ and $\psi_{2 n}$ be as defined in (3.14) and (3.15). Let $d_{n}$ be as in (3.27). Assume that Condition (F1) of Theorem 9.2.1 holds. 
Let

$$
L_{n}(s)=\left[\exp \left(z_{n}\right) \frac{\psi_{2 n}^{\prime}\left(-<\tau_{n}, \tau_{n}+i \frac{s}{\sqrt{a_{n}}}>\right)}{\psi_{2 n}^{\prime}\left(-<\tau_{n}, \tau_{n}>\right)}-1-z_{n}\right]
$$

where

$$
\begin{aligned}
z_{n}(s)= & {\left[\frac{-i}{\sqrt{a_{n}}} \sum_{|\alpha|=3} a_{\alpha}^{(n)} s^{\alpha}+\frac{i\left(<r_{n}, s>\right)^{3}}{6 \sqrt{a_{n}}} \psi_{2 n}^{\prime \prime \prime}\left(-<r_{n}, s>\right)\right.} \\
& \left.+a_{n} R_{1 n}\left(\tau_{n}+i \frac{s}{\sqrt{a_{n}}}\right)+a_{n} R_{2 n}\left(-<r_{n}, \tau_{n}+i \frac{s}{\sqrt{a_{n}}}>\right)\right] .
\end{aligned}
$$

Then, there exists $\delta>0$ such that

$$
\left[\frac{\left|a_{n}\right|}{(2 \pi)^{k}}\right]^{1 / 2} \int_{|s|<\sqrt{a_{n}} \delta} \exp \left(\frac{-s^{\prime} d_{n} s}{2}\right) L_{n}(s) d s=O\left(1 / a_{n}\right) .
$$

Proof. Consider

$$
\begin{aligned}
Q_{n}^{*}= & {\left[\frac{\left|d_{n}\right|}{(2 \pi)^{k}}\right]^{1 / 2} \int_{|s|<\sqrt{a_{n}} \delta} \exp \left(\frac{-s^{\prime} d_{n} s}{2}\right) } \\
& {\left[\exp \left(z_{n}\right) \frac{\psi_{2 n}^{\prime}\left(-<r_{n}, \tau_{n}+i \frac{s}{\sqrt{a_{n}}}>\right)}{\psi_{2 n}^{\prime}\left(-<r_{n}, \tau_{n}>\right)}-1-z_{n}\right] d s }
\end{aligned}
$$

for $\delta>0$. The r.h.s. of the above equation can be written as the sum of four integrals as follows:

$$
\begin{aligned}
& Q_{n}^{*}=\left[\frac{\left|d_{n}\right|}{(2 \pi)^{k}}\right]^{1 / 2} \int_{|s|<\sqrt{a_{n}} \delta} \exp \left(\frac{-s^{\prime} d_{n} s}{2}\right)\left[\left(\exp \left(z_{n}\right)-1-z_{n}\right)\right. \\
& \quad \times \frac{\psi_{2 n}^{\prime}\left(-<r_{n}, \tau_{n}+i \frac{s}{\sqrt{a_{n}}}>\right)}{\psi_{2 n}^{\prime}\left(-<r_{n}, \tau_{n}>\right)}+\left(\frac{\psi_{2 n}^{\prime}\left(-<r_{n}, \tau_{n}+i \frac{s}{\sqrt{a_{n}}}>\right)}{\psi_{2 n}^{\prime}\left(-<r_{n}, \tau_{n}>\right)}-1\right) \\
&\left.-\left(z_{n}\right)+\left(z_{n} \frac{\psi_{2 n}^{\prime}\left(-<r_{n}, \tau_{n}+i \frac{s}{\sqrt{a_{n}}}>\right)}{\psi_{2 n}^{\prime}\left(-<r_{n}, \tau_{n}>\right)}\right)\right] d s \\
&= I_{n 1}^{\prime}+I_{n 2}^{\prime}+I_{n 3}^{\prime}+I_{n 4}^{\prime}(s a y)
\end{aligned}
$$


where

$$
\begin{gathered}
I_{n 1}^{\prime}=\left[\frac{\left|d_{n}\right|}{(2 \pi)^{k}}\right]^{1 / 2} \int_{|s|<\sqrt{a_{n}} \delta} \exp \left(\frac{-s^{\prime} d_{n} s}{2}\right)\left(\exp \left(z_{n}\right)-1-z_{n}\right) \\
\frac{\psi_{2 n}^{\prime}\left(-<r_{n}, \tau_{n}+i \frac{s}{\sqrt{a_{n}}}>\right)}{\psi_{2 n}^{\prime}\left(-<r_{n}, \tau_{n}>\right)} d s . \\
I_{n 2}^{\prime}=\left[\frac{\left|d_{n}\right|}{(2 \pi)^{k}}\right]^{1 / 2} \int_{|s|<\sqrt{a_{n}} \delta} \exp \left(\frac{-s^{\prime} d_{n} s}{2}\right)\left(\frac{\psi_{2 n}^{\prime}\left(-<r_{n}, \tau_{n}+i \frac{s}{\sqrt{a_{n}}}>\right)}{\psi_{2 n}^{\prime}\left(-<r_{n}, \tau_{n}>\right)}-1\right) d s . \\
I_{n 3}^{\prime}=\left[\frac{\left|d_{n}\right|}{(2 \pi)^{k}}\right]^{1 / 2} \int_{|s|<\sqrt{a_{n}} \delta} \exp \left(\frac{-s^{\prime} d_{n} s}{2}\right)\left(-z_{n}\right) d s . \\
I_{n 4}^{\prime}=\left[\frac{\left|d_{n}\right|}{(2 \pi)^{k}}\right]^{1 / 2} \int_{|s|<\sqrt{a_{n}} \delta} \exp \left(\frac{-s^{\prime} d_{n} s}{2}\right) z_{n} \frac{\psi_{2 n}^{\prime}\left(-<r_{n}, \tau_{n}+i \frac{s}{\sqrt{a_{n}}}>\right)}{\psi_{2 n}^{\prime}\left(-<r_{n}, \tau_{n}>\right)} d s .
\end{gathered}
$$

We complete the proof of this lemma by showing $I_{n i}^{\prime}=O\left(1 / a_{n}\right), i=$ $1,2,3,4$. To show that $I_{n 1}^{\prime}=O\left(1 / a_{n}\right)$, we get an upperbound for $\left(\exp \left(z_{n}\right)-\right.$ $\left.1-z_{n}\right)$.

For $|s|<\sqrt{a_{n}} \delta$, consider

$$
\begin{aligned}
\left|z_{n}\right|= & \mid \frac{-i}{\sqrt{a_{n}}} \sum_{|\alpha|=3} a_{\alpha}^{(n)} s^{\alpha}+\frac{i\left(<r_{n}, s>\right)^{3}}{6 \sqrt{a_{n}}} \psi_{2 n}^{\prime \prime \prime}\left(-<r_{n}, s>\right) \\
& +a_{n} R_{1 n}\left(\tau_{n}+i \frac{s}{\sqrt{a_{n}}}\right)+a_{n} R_{2 n}\left(-<r_{n}, \tau_{n}+i \frac{s}{\sqrt{a_{n}}}>\right) \mid .
\end{aligned}
$$

The bound on $\left|R_{2 n}\left(-<r_{\pi}, \tau_{n}+i t>\right)\right|$ given in Remark 3.2 .5 yields yields

$$
\left|z_{n}\right| \leq\left[\frac{1}{\sqrt{a_{n}}} \frac{\beta_{1}|s|^{3}}{\left(c_{1}-b_{1}\right)^{3}}+\frac{|s|^{3}}{\sqrt{a_{n}}} \frac{\beta_{2}\left(\sum_{j=1}^{k} \overline{r_{j}}\right)^{3}}{\left(c_{2}-b_{2}\right)^{3}}\right.
$$




$$
\begin{gathered}
\left.+\frac{2 \beta_{1}|s|^{4}}{a_{n}\left(c_{1}-b_{1}\right)^{4}}+\frac{2|s|^{4} \beta_{2}\left(\sum_{j=1}^{k} \overline{r_{j}}\right)^{4}}{a_{n}\left(c_{2}-b_{2}\right)^{4}}\right]^{2} \exp \left(M(\delta)|s|^{2}\right) \\
\leq \frac{\beta_{1} \delta|s|^{2}}{\left(c_{1}-b_{1}\right)^{3}}+\frac{\beta_{2} \delta|s|^{2}\left(\sum_{j=1}^{k} \overline{r_{j}}\right)^{3}}{\left(c_{2}-b_{2}\right)^{3}} \\
+\frac{2 \beta_{1} \delta^{2}|s|^{2}}{\left(c_{1}-b_{1}\right)^{4}}+\frac{2 \beta_{2} \delta^{2}|s|^{2}\left(\sum_{j=1}^{k} \overline{r_{j}}\right)^{4}}{\left(c_{2}-b_{2}\right)^{4}} \\
\leq\left[\frac{\beta_{1} \delta}{\left(c_{1}-b_{1}\right)^{3}}+\frac{\beta_{2} \delta\left(\sum_{j=1}^{k} \overline{r_{j}}\right)^{3}}{\left(c_{2}-b_{2}\right)^{3}}\right. \\
\left.+\frac{2 \beta_{1} \delta^{2}}{\left(c_{1}-b_{1}\right)^{4}}+\frac{2 \beta_{2} \delta^{2}\left(\sum_{j=1}^{k} \overline{r_{j}}\right)^{4}}{\left(c_{2}-b_{2}\right)^{4}}\right]|s|^{2}
\end{gathered}
$$

wherein we have used the fact that $\left|r_{j n}\right|<\overline{r_{j}}$ Thus we have

$$
\left|z_{n}(s)\right|<M(\delta)|s|^{2}
$$

where

$$
\begin{aligned}
M(\delta)= & {\left[\frac{\beta_{1} \delta}{\left(c_{1}-b_{1}\right)^{3}}+\frac{\beta_{2} \delta\left(\sum_{j=1}^{k} \overline{r_{j}}\right)^{3}}{\left(c_{2}-b_{2}\right)^{3}}\right.} \\
& \left.+\frac{2 \beta_{1} \delta^{2}}{\left(c_{1}-b_{1}\right)^{4}}+\frac{2 \beta_{2} \delta^{2}\left(\sum_{j=1}^{k} \overline{r_{j}}\right)^{4}}{\left(c_{2}-b_{2}\right)^{4}}\right] .
\end{aligned}
$$

Let us choose our $\delta$ such that $M(\delta)<\alpha_{1} / 2 k$. Using the simple inequality, $\left|\exp \left(z_{n}\right)-1-z_{n}\right| \leq\left|z_{n}\right|^{2} \exp \left(\left|z_{n}\right|\right)$, we obtain,

$$
\begin{aligned}
\left|\exp \left(z_{n}\right)-1-z_{n}\right| \leq & {\left[\frac{1}{\sqrt{a_{n}}} \frac{\beta_{1}|s|^{3}}{\left(c_{1}-b_{1}\right)^{3}}+\frac{|s|^{3}}{\sqrt{a_{n}}} \frac{\beta_{2}\left(\sum_{j=1}^{k} \overline{r_{j}}\right)^{3}}{\left(c_{2}-b_{2}\right)^{3}}\right.} \\
& \left.+\frac{2 \beta_{1}|s|^{4}}{a_{n}\left(c_{1}-b_{1}\right)^{4}}+\frac{2|s|^{4} \beta_{2}\left(\sum_{j=1}^{k} \overline{r_{j}}\right)^{4}}{a_{n}\left(c_{2}-b_{2}\right)^{4}}\right]^{2} \exp \left(M(\delta)|s|^{2}\right)
\end{aligned}
$$


Consider

$$
\begin{gathered}
\left|I_{n 1}^{\prime}\right|=\mid\left[\frac{\left|d_{n}\right|}{(2 \pi)^{k}}\right]^{1 / 2} \int_{|s|<\sqrt{a_{n}} \delta} \exp \left(\frac{-s^{\prime} d_{n} s}{2}\right)\left(\exp \left(z_{n}\right)-1-z_{n}\right) \\
\quad \times \frac{\psi_{2 n}^{\prime}\left(-<r_{n}, \tau_{n}+i \frac{s}{\sqrt{a_{n}}}>\right)}{\psi_{2 n}^{\prime}\left(-<r_{n}, \tau_{n}>\right)} d s \mid .
\end{gathered}
$$

Substituting the bound in (4.46) in the above equation, we get

$$
\begin{aligned}
\left|I_{n 1}^{\prime}\right| \leq & {\left[\frac{\left|d_{n}\right|}{(2 \pi)^{k}}\right]^{1 / 2} \int_{|s|<\sqrt{a_{n}} \delta} \exp \left(\frac{-s^{\prime} d_{n} s}{2}\right) \exp \left(M(\delta)|s|^{2}\right) } \\
& \times\left[\frac{1}{\sqrt{a_{n}}} \frac{\beta_{1}|s|^{3}}{\left(c_{1}-b_{1}\right)^{3}}+\frac{|s|^{3}}{\sqrt{a_{n}}} \frac{\beta_{2}\left(\sum_{j=1}^{k} \overline{r_{j}}\right)^{3}}{\left(c_{2}-b_{2}\right)^{3}}+\frac{2 \beta_{1}|s|^{4}}{a_{n}\left(c_{1}-b_{1}\right)^{4}}\right. \\
& \left.+\frac{2|s|^{4} \beta_{2}\left(\sum_{j=1}^{k} \overline{r_{j}}\right)^{4}}{a_{n}\left(c_{2}-b_{2}\right)^{4}}\right]^{2}\left|\frac{\psi_{2 n}^{\prime}\left(-<r_{n}, \tau_{n}+i \frac{s}{\sqrt{a_{n}}}>\right)}{\psi_{2 n}^{\prime}\left(-<r_{n}, \tau_{n}>\right)}\right| d s \\
\leq & \frac{1}{a_{n}}\left[\frac{\left|d_{n}\right|}{(2 \pi)^{k}}\right]^{1 / 2} \int_{|s|<\sqrt{a_{n} \delta}} \exp \left(\frac{-\alpha_{1} s^{\prime} s}{2}\right) \exp \left(M(\delta)|s|^{2}\right) \\
& \times\left[\frac{\beta_{1}|s|^{3}}{\left(c_{1}-b_{1}\right)^{3}}+\frac{|s|^{3} \beta_{2}\left(\sum_{j=1}^{k} \overline{r_{j}}\right)^{3}}{\left(c_{2}-b_{2}\right)^{3}}+\frac{2 \beta_{1}|s|^{4}}{\sqrt{a_{n}}\left(c_{1}-b_{1}\right)^{4}}\right. \\
& \left.+\frac{2|s|^{4} \beta_{2}\left(\sum_{j=1}^{k} \overline{r_{j}}\right)^{4}}{\sqrt{a_{n}}\left(c_{2}-b_{2}\right)^{4}}\right]^{2}\left|\frac{\psi_{2 n}^{\prime}\left(-<r_{n}, \tau_{n}+i \frac{s}{\sqrt{a_{n}}}>\right)}{\psi_{2 n}^{\prime}\left(-<r_{n}, \tau_{n}>\right)}\right| d s .
\end{aligned}
$$

Since $k\left(s^{\prime} s\right) \geq|s|^{2}$, we have

$$
\begin{aligned}
\left|I_{n 1}^{\prime}\right| \leq \frac{1}{a_{n}}\left[\frac{\left|d_{n}\right|}{(2 \pi)^{k}}\right]^{1 / 2} \int_{|s|<\sqrt{a_{n}} \delta} \exp \left(-\alpha_{1} \frac{s^{\prime} s}{2}+M(\delta) k\left(s^{\prime} s\right)\right) \\
\times\left[\frac{\beta_{1}|s|^{3}}{\left(c_{1}-b_{1}\right)^{3}}+\frac{|s|^{3} \beta_{2}\left(\sum_{j=1}^{k} \overline{\gamma_{j}}\right)^{3}}{\left(c_{2}-b_{2}\right)^{3}}+\frac{2 \beta_{1}|s|^{4}}{\sqrt{a_{n}}\left(c_{1}-b_{1}\right)^{4}}\right.
\end{aligned}
$$




$$
\begin{gathered}
\left.+\frac{2|s|^{4} \beta_{2}\left(\sum_{j=1}^{k} \overline{r_{j}}\right)^{4}}{\sqrt{a_{n}}\left(c_{2}-b_{2}\right)^{4}}\right]^{2}\left|\frac{\psi_{2 n}^{\prime}\left(-<r_{n}, \tau_{n}+i \frac{s}{\sqrt{a_{n}}}>\right)}{\psi_{2 n}^{\prime}\left(-<r_{n}, \tau_{n}>\right)}\right| d s \\
\leq \frac{1}{a_{n}}\left[\frac{\left|d_{n}\right|}{(2 \pi)^{k}}\right]^{1 / 2} \int_{|s|<\sqrt{a_{n}} \delta} \exp \left(-\frac{s^{\prime} s}{2}\left(\alpha_{1}-2 k M(\delta)\right)\right) \\
\quad \times\left[\frac{\beta_{1}|s|^{3}}{\left(c_{1}-b_{1}\right)^{3}}+\frac{|s|^{3} \beta_{2}\left(\sum_{j=1}^{k} \overline{r_{j}}\right)^{3}}{\left(c_{2}-b_{2}\right)^{3}}+\frac{2 \beta_{1}|s|^{4}}{\sqrt{a_{n}}\left(c_{1}-b_{1}\right)^{4}}\right. \\
\left.+\frac{2|s|^{4} \beta_{2}\left(\sum_{j=1}^{k} \overline{r_{j}}\right)^{4}}{\sqrt{a_{n}}\left(c_{2}-b_{2}\right)^{4}}\right]^{2}\left|\frac{\psi_{2 n}^{\prime}\left(-<\tau_{n}, \tau_{n}+i \frac{s}{\sqrt{a_{n}}}>\right)}{\psi_{2 n}^{\prime}\left(-<r_{n}, \tau_{n}>\right)}\right| d s .
\end{gathered}
$$

Proceeding as in the univariate case we can show that

$$
\left|\psi_{2 n}^{\prime}\left(-<r_{n}, \tau_{n}+i t>\right) / \psi_{2 n}^{\prime}\left(-<r_{n}, \tau_{n}>\right)\right|
$$

is bounded in $\mathrm{n}$. Since $M(\delta)<\alpha_{1} / 2 k$, the inequality (3.45) shows that $I_{n 1}^{\prime}=O\left(1 / a_{n}\right)$. Next consider,

$$
\begin{aligned}
\left|I_{n 2}^{\prime}\right|= & {\left[\frac{\left|d_{n}\right|}{(2 \pi)^{k}}\right]^{1 / 2} \int_{|s|<\sqrt{a_{n}} \delta} \exp \left(\frac{-s^{\prime} d_{n} s}{2}\right)\left(\frac{\psi_{2 n}^{\prime}\left(-<r_{n}, \tau_{n}+i \frac{s}{\sqrt{a_{n}}}>\right)}{\psi_{2 n}^{\prime}\left(-<r_{n}, \tau_{n}>\right)}-1\right) d s } \\
= & {\left[\frac{\left|d_{n}\right|}{(2 \pi)^{k}}\right]^{1 / 2} \int_{|s|<\sqrt{a_{n}} \delta} \exp \left(\frac{-s^{\prime} d_{n} s}{2}\right)\left[-\frac{i<r_{n}, s>}{a_{n}} \frac{\psi_{2 n}^{\prime \prime}\left(-<r_{n}, \tau_{n}>\right)}{\psi_{2 n}^{\prime}\left(-<r_{n}, \tau_{n}>\right)}\right.} \\
& \left.-\frac{<r_{n}, s>^{2}}{a_{n}} \frac{\psi_{2 n}^{\prime \prime \prime}\left(-<r_{n}, \tau_{n}>\right)}{\psi_{2 n}^{\prime}\left(-<r_{n}, \tau_{n}>\right)}+\frac{R\left(<r_{n}, \tau_{n}+i \frac{s}{a_{n}}>\right)}{\psi_{2 n}^{\prime}\left(-<r_{n}, \tau_{n}>\right)}\right] d s .
\end{aligned}
$$

It is easy to see that the first integral on the right hand side of $I_{n 2}^{\prime}$ is zero, second integral is $O\left(1 / a_{n}\right)$. We can use the bound on $\left|R\left(<r_{n}, \tau_{n}+i \frac{s}{a_{n}}>\right)\right|$ (see Remark 3.2.6) to show that the third integral is $O\left(1 / a_{n}\right)$.

Therefore

$$
I_{n 2}^{\prime}=O\left(1 / a_{n}\right)
$$


Now, consider

$$
\begin{aligned}
&\left|I_{n 3}^{\prime}\right|= {\left[\frac{\left|d_{n}\right|}{(2 \pi)^{k}}\right]^{1 / 2} \int_{|s|<\sqrt{a_{n}} \delta} \exp \left(\frac{-s^{\prime} d_{n} s}{2}\right)\left(-z_{n}\right) d s } \\
&=(-1)\left[\frac{\left|d_{n}\right|}{(2 \pi)^{k}}\right]^{1 / 2} \int_{|s|<\sqrt{a_{n}} \delta} \exp \left(\frac{-s^{\prime} d_{n} s}{2}\right) \\
& {\left[\frac{-i}{\sqrt{a_{n}}} \sum_{|\alpha|=3} a_{\alpha}^{(n)} s^{\alpha}+\frac{i<r_{n}, s>^{3}}{6 \sqrt{a_{n}}} \psi_{2 n}^{\prime \prime \prime}\left(-<r_{n}, \tau_{n}>\right)\right.} \\
&\left.+a_{n} R_{1 n}\left(\tau_{n}+i \frac{s}{\sqrt{a_{n}}}\right)+a_{n} R_{2 n}\left(-<r_{n}, \tau_{n}+i \frac{s}{\sqrt{a_{n}}}>\right)\right] d s .
\end{aligned}
$$

The first two integrals on the right hand side of $I_{n 3}^{\prime}$ are zero. We use the bounds on $R_{1 n}(\tau+i t)$ and $R_{2 n}(\tau+i t)$, obtained in Lemma 3.2.4 and Remark 3.2 .5 , to show that the third and fourth integrals are $O\left(1 / a_{n}\right)$.

Thus

$$
I_{n 3}^{\prime}=O\left(1 / a_{n}\right)
$$

Lastly, consider

$$
\begin{aligned}
I_{n 4}^{\prime}= & {\left[\frac{\left|d_{n}\right|}{(2 \pi)^{k}}\right]^{1 / 2} \int_{|s|<\sqrt{a_{n}} \delta} \exp \left(\frac{-s^{\prime} d_{n} s}{2}\right) z_{n} \frac{\psi_{2 n}^{\prime}\left(-<r_{n}, \tau_{n}+i \frac{s}{\sqrt{a_{n}}}>\right)}{\psi_{2 n}^{\prime}\left(-<r_{n}, \tau_{n}>\right)} d s } \\
= & {\left[\frac{\left|d_{n}\right|}{(2 \pi)^{k}}\right]^{1 / 2} \int_{|s|<\sqrt{a_{n}} \delta} \exp \left(\frac{-s^{\prime} d_{n} s}{2}\right)\left[\frac{-i}{\sqrt{a_{n}}} \sum_{|\alpha|=3} a_{\alpha}^{(n)} s^{\alpha}\right.} \\
& +\frac{i<r_{n}, s>^{3}}{6 \sqrt{a_{n}}} \psi_{2 n}^{\prime \prime \prime}\left(-<r_{n}, \tau_{n}>\right)+a_{n} R_{1 n}\left(\tau_{n}+i \frac{s}{\sqrt{a_{n}}}\right) \\
& \left.+a_{n} R_{2 n}\left(-<r_{n}, \tau_{n}+i \frac{s}{\sqrt{a_{n}}}>\right)\right]
\end{aligned}
$$




$$
\begin{gathered}
{\left[1-\frac{i<r_{n}, s>}{a_{n}} \frac{\psi_{2 n}^{\prime \prime}\left(-<r_{n}, \tau_{n}>\right)}{\psi_{2 n}^{\prime}\left(-<r_{n}, \tau_{n}>\right)}-\frac{<r_{n}, s>^{2}}{a_{n}} \frac{\psi_{2 n}^{\prime \prime \prime}\left(-<r_{n}, \tau_{n}>\right)}{\psi_{2 n}^{\prime}\left(-<r_{n}, \tau_{n}>\right)}\right.} \\
\left.+\frac{R\left(<r_{n}, \tau_{n}+i \frac{s}{a_{n}}>\right)}{\psi_{2 n}^{\prime}\left(-<r_{n}, \tau_{n}>\right)}\right] d s .
\end{gathered}
$$

Using the argument similar to the univariate case the term $I_{n 4}^{\prime}$ can be easily verified to be equal to $O\left(1 / a_{n}\right)$. This completes the proof of Lemma 3.2.6.

The next lemma shows that the term $I_{n 2}$ appearing in the proof of the main theorem is $1+O\left(1 / a_{n}\right)$.

Lemma 3.2.9 Let $f_{n}(t)$ be as defined in (\$.\$1). Let $\delta>0$ as in Lemma \$.2.8. Then

$$
\begin{aligned}
I_{n 2} & =\left[\frac{a_{n}^{k}\left|d_{n}\right|}{(2 \pi)^{k}}\right]^{1 / 2} \int_{|t|<\delta} \exp \left(\left[a_{n}\left(f_{n}\left(\tau_{n}+i t\right)-f_{n}\left(\tau_{n}\right)\right)\right]\right) \frac{\psi_{2 n}^{\prime}\left(-<r_{n}, \tau_{n}+i t>\right)}{\psi_{2 n}^{\prime}\left(-<r_{n}, \tau_{n}>\right)} d t \\
& =1+O\left(1 / a_{n}\right) .
\end{aligned}
$$

Proof. Consider

$$
\begin{aligned}
I_{n 2}= & {\left[\frac{a_{n}^{k}\left|d_{n}\right|}{(2 \pi)^{k}}\right]^{1 / 2} \int_{|t|<\delta} \exp \left(a _ { n } \left[\psi_{1 n}\left(\tau_{n}+i t\right)-\psi_{1 n}\left(\tau_{n}\right)\right.\right.} \\
& \left.\left.+\psi_{2 n}\left(-<\tau_{n}, \tau_{n}+i t>\right)-\psi_{2 n}\left(-<r_{n}, \tau_{n}>\right)\right]\right) \frac{\psi_{2 n}^{\prime}\left(-<r_{n}, \tau_{n}+i t>\right)}{\psi_{2 n}^{\prime}\left(-<r_{n}, \tau_{n}>\right)} d t
\end{aligned}
$$

By making a change of variable $t=s / \sqrt{a_{n}}$, we have,

$$
\begin{aligned}
I_{n 2}= & {\left[\frac{\left|d_{n}\right|}{(2 \pi)^{k}}\right]^{1 / 2} \int_{|s|<\sqrt{a_{n}} \delta} \exp \left(a _ { n } \left[\psi_{1 n}\left(\tau_{n}+i \frac{s}{\sqrt{a_{n}}}\right)-\psi_{1 n}\left(\tau_{n}\right)\right.\right.} \\
& \left.\left.+\psi_{2 n}\left(-<r_{n}, \tau_{n}+i \frac{s}{\sqrt{a_{n}}}>\right)-\psi_{2 n}\left(-<r_{n}, \tau_{n}>\right)\right]\right) \frac{\psi_{2 n}^{\prime}\left(-<r_{n}, \tau_{n}+i \frac{s}{\sqrt{a_{n}}}>\right)}{\psi_{2 n}^{\prime}\left(-<r_{n}, \tau_{n}>\right)} d s .
\end{aligned}
$$


For $|s|<\sqrt{a_{n}} \delta$, we use Taylor's series for $\psi_{1 n}$ and $\psi_{2 n}$ to get

$$
\begin{aligned}
I_{n 2}= & {\left[\frac{\left|d_{n}\right|}{(2 \pi)^{k}}\right]^{1 / 2} \int_{|s|<\sqrt{a_{n}} \delta} \exp \left(-a_{n}\left[\frac{1}{2 a_{n}} s^{\prime} \nabla^{2} \psi_{1 n}\left(\tau_{n}\right) s\right.\right.} \\
& +\frac{<r_{n}, s>^{2}}{2 a_{n}} \psi_{2 n}^{\prime \prime}\left(-<r_{n}, \tau_{n}>\right)-\frac{i}{a_{n} \sqrt{a_{n}}} \sum_{|\alpha|=3} a_{\alpha}^{(n)} s^{\alpha} \\
& -\frac{i<r_{n}, s>^{3}}{6 a_{n} \sqrt{a_{n}}} \psi_{2 n}^{\prime \prime \prime}\left(-<r_{n}, \tau_{n}>\right)-R_{1 n}\left(\tau_{n}+i \frac{s}{\sqrt{a_{n}}}\right) \\
& \left.\left.-R_{2 n}\left(-<r_{n}, \tau_{n}+i \frac{s}{\sqrt{a_{n}}}>\right)\right]\right) \frac{\psi_{2 n}^{\prime}\left(-<r_{n}, \tau_{n}+i \frac{s}{\sqrt{a_{n}}}>\right)}{\psi_{2 n}^{\prime}\left(-<r_{n}, \tau_{n}>\right)} d s .
\end{aligned}
$$

We note that $s^{\prime} \nabla^{2} \psi_{1 n}\left(\tau_{n}\right) s+<r_{n}, s>^{2} \psi_{2 n}^{\prime \prime}\left(-<r_{n}, \tau_{n}>\right)=s^{\prime} d_{n} s$. Thus we have,

$$
\begin{aligned}
\left|I_{n 2}\right|= & {\left[\frac{\left|d_{n}\right|}{(2 \pi)^{k}}\right]^{1 / 2} \int_{|s|<\sqrt{a_{n}} \delta} \exp \left(\frac{-s^{\prime} d_{n} s}{2}\right) \exp \left(-\frac{i}{\sqrt{a_{n}}} \sum_{|\alpha|=3} a_{\alpha}^{(n)} s^{\alpha}\right.} \\
& -\frac{i<r_{n}, s>^{3}}{6 \sqrt{a_{n}}} \psi_{2 n}^{\prime \prime \prime}\left(-<r_{n}, \tau_{n}>\right)+a_{n} R_{1 n}\left(\tau_{n}+i \frac{s}{\sqrt{a_{n}}}\right) \\
& \left.+a_{n} R_{2 n}\left(-<r_{n}, \tau_{n}+i \frac{s}{\sqrt{a_{n}}}>\right)\right) \frac{\psi_{2 n}^{\prime}\left(-<r_{n}, \tau_{n}+i \frac{s}{\sqrt{a_{n}}}>\right)}{\psi_{2 n}^{\prime}\left(-<r_{n}, \tau_{n}>\right)} d s \\
= & {\left[\frac{\left|d_{n}\right|}{(2 \pi)^{k}}\right]^{1 / 2} \int_{|s|<\sqrt{a_{n}} \delta} \exp \left(\frac{-s^{\prime} d_{n} s}{2}\right)\left[1-\frac{i}{\sqrt{a_{n}}} \sum_{|\alpha|=3} a_{\alpha}^{(n)} s^{\alpha}\right.} \\
& -\frac{i<r_{n}, s>3}{6 \sqrt{a_{n}}} \psi_{2 n}^{\prime \prime \prime}\left(-<r_{n}, \tau_{n}>\right)+a_{n} R_{1 n}\left(\tau_{n}+i \frac{s}{\sqrt{a_{n}}}\right) \\
& \left.+a_{n} R_{2 n}\left(-<r_{n}, \tau_{n}+i \frac{s}{\sqrt{a_{n}}}>\right)+L_{n}(s)\right] d s
\end{aligned}
$$


where $d_{n}, L_{n}(s)$ and $z_{n}(s)$ are as defined in (3.30), (3.35) and (3.36) respectively. The right hand side of (3.56) involves six integrals. The $1^{\text {st }}$ integral is $1+o\left(1 / a_{n}\right)$. Trivially, $2^{\text {nd }}$ and $3^{r d}$ integrals are equal to zero. In order to show that the $4^{\text {th }}$ integral is $1+O\left(1 / a_{n}\right)$, we use the bound on $R_{1 n}\left(\tau_{n}+i s / \sqrt{a_{n}}\right)$ given in Lemma 3.2.4. Thus

$$
\begin{aligned}
& \left|\left[\frac{\left|d_{n}\right|}{(2 \pi)^{k}}\right]^{1 / 2} \int_{|s|<\sqrt{a_{n}} \delta} a_{n} R_{1 n}\left(\tau_{n}+i \frac{s}{\sqrt{a_{n}}}\right) \exp \left(\frac{-s^{\prime} d_{n} s}{2}\right) d s\right| \\
\leq & a_{n}\left[\frac{\left|d_{n}\right|}{(2 \pi)^{k}}\right]^{1 / 2} \int_{|s|<\sqrt{a_{n}} \delta} \exp \left(\frac{-s^{\prime} d_{n} s}{2}\right)\left|R_{1 n}\left(\tau_{n}+i \frac{s}{\sqrt{a_{n}}}\right)\right| d s \\
\leq & \frac{1}{a_{n}}\left[\frac{\left|d_{n}\right|}{(2 \pi)^{k}}\right]^{1 / 2} \frac{2 \beta_{1}}{\left(c_{1}-b_{1}\right)^{4}} \int_{|s|<\sqrt{a_{n}} \delta}|s|^{4} \exp \left(\frac{-s^{\prime} d_{n} s}{2}\right) d s \\
= & O\left(1 / a_{n}\right) .
\end{aligned}
$$

Similarly, the $5^{\text {th }}$ integral can be shown to be equal to $O\left(1 / a_{n}\right)$. The $6^{\text {th }}$ integral, $\left[\left|d_{n}\right| /(2 \pi)^{k}\right]^{1 / 2} \int_{|s|<\sqrt{a_{n}} \delta} L_{n}(s) \exp \left(-s^{\prime} d_{n} s / 2\right) d s$ is $Q_{n}^{*}$ defined in (3.37). Therefore, it follows from Lemma 3.2.8 that

$$
\left[\frac{\left|d_{n}\right|}{(2 \pi)^{k}}\right]^{1 / 2} \int_{|s|<\sqrt{a_{n}} \delta} L_{n}(s) \exp \left(\frac{-s^{\prime} d_{n} s}{2}\right) d s=O\left(1 / a_{n}\right)
$$

Thus

$$
I_{n 2}=1+O\left(1 / a_{n}\right)
$$

We now proceed with the proof of main Theorem.

Proof. Let $G_{n}$ be the distribution function of $R_{n}=T_{n} / S_{n}$. Then, for $r=\left(r_{1}, r_{2}, \cdots, \tau_{k}\right)$

$$
G_{n}(r)=\operatorname{Pr}\left[\frac{T_{1 n}}{S_{n}} \leq r_{1}, \ldots, \frac{T_{k n}}{S_{n}} \leq r_{k}\right]
$$




$$
\begin{aligned}
& =\int_{0}^{\infty} \operatorname{Pr}\left[T_{1 n} \leq r_{1} y, \ldots, T_{k n} \leq r_{k} y\right] d F_{2 n}(y) \\
& =\int_{0}^{\infty} F_{1 n}(r y) d F_{2 n}(y) .
\end{aligned}
$$

The density function $g_{n}$ of $G_{n}$ is given by

$$
g_{n}(r)=\int_{0}^{\infty} y^{k} f_{1 n}(r y) d F_{2 n}(y)
$$

where $f_{1 n}$ is the p.d.f. of $T_{n}$.

Using the conjugate distribution technique, we get,

$$
f_{1 n}(r y)=\left[\frac{1}{2 \pi}\right]^{k} \int_{R^{k}} \phi_{1 n}(\tau+i t) \exp (-<r y, \tau+i t>) d t
$$

Therefore,

$$
\begin{aligned}
g_{n}(r) & =\int_{0}^{\infty} y^{k}\left[\frac{1}{2 \pi}\right]^{k} \int_{R^{k}} \phi_{1 n}(\tau+i t) \exp (-<r y, \tau+i t>) d t d F_{2 n}(y) \\
& =\left[\frac{1}{2 \pi}\right]^{k} \int_{R^{k}} \phi_{1 n}(\tau+i t)\left[\int_{0}^{\infty} y^{k} \exp (-<r y, \tau+i t>) d F_{2 n}(y)\right] d t \\
& =\left[\frac{1}{2 \pi}\right]^{k} \int_{R^{k}} \phi_{1 n}(\tau+i t)\left[\int_{0}^{\infty} y^{k} \exp (-<r y, \tau+i t>) d F_{2 n}(y)\right] d t \\
& =\left[\frac{1}{2 \pi}\right]^{k} \int_{R^{k}} \phi_{1 n}(\tau+i t) \phi_{2 n}^{\prime}(-<r, \tau+i t>) d t \\
& =\left[\frac{a_{n}}{2 \pi}\right]^{k} \int_{R^{k}} \exp \left(a_{n}\left[\psi_{1 n}(\tau+i t)+\psi_{2 n}(-<r, \tau+i t>)\right]\right) \psi_{2 n}^{\prime}(-<r, \tau+i t>) d t
\end{aligned}
$$

We now replace $r$ by $r_{n}$ and $\tau$ by $\tau_{n}$ to get

$g_{n}\left(r_{n}\right)=\left[\frac{a_{n}}{2 \pi}\right]^{k} \int_{R^{k}} \exp \left(a_{n}\left[\psi_{1 n}\left(\tau_{n}+i t\right)+\psi_{2 n}\left(-<r_{n}, \tau_{n}+i t>\right)\right]\right)$ 


$$
\begin{aligned}
& \times \psi_{2 n}^{\prime}\left(-<r_{n}, \tau_{n}+i t>\right) d t \\
= & {\left[\frac{a_{n}}{2 \pi}\right]^{k / 2} \frac{\psi_{2 n}^{\prime}\left(-<r_{n}, \tau_{n}>\right)}{\left|d_{n}\right|^{1 / 2}} \exp \left(a_{n}\left[\psi_{1 n}\left(\tau_{n}\right)+\psi_{2 n}\left(-<r_{n}, \tau_{n}>\right)\right]\right) I_{n} }
\end{aligned}
$$

where

$$
\begin{aligned}
I_{n}= & {\left[\frac{a_{n}^{k}\left|d_{n}\right|}{(2 \pi)^{k}}\right]^{1 / 2} \int_{R^{k}} \exp \left(a _ { n } \left[\psi_{1 n}\left(\tau_{n}+i t\right)-\psi_{1 n}\left(\tau_{n}\right)\right.\right.} \\
& \left.\left.+\psi_{2 n}\left(-<r_{n}, \tau_{n}+i t>\right)-\psi_{2 n}\left(-<r_{n}, \tau_{n}>\right)\right]\right) \frac{\psi_{2 n}^{t}\left(-<r_{n}, \tau_{n}+i t>\right)}{\psi_{2 n}^{\prime}\left(-<r_{n}, \tau_{n}>\right)} d t .
\end{aligned}
$$

Writing $\psi_{1 n}(t)$ and $\psi_{2 n}(t)$ in terms of $f_{n}(t)$ (see (3.31)) we get,

$$
\begin{aligned}
I_{n}= & {\left[\frac{a_{n}^{k}\left|d_{n}\right|}{(2 \pi)^{k}}\right]^{1 / 2} \int_{R^{k}} \exp \left(a_{n}\left[f_{n}\left(\tau_{n}+i t\right)-f_{n}\left(\tau_{n}\right)\right]\right) \frac{\psi_{2 n}^{\prime}\left(-<r_{n}, \tau_{n}+i t>\right)}{\psi_{2 n}^{\prime}\left(-<r_{n}, \tau_{n}>\right)} d t } \\
= & {\left[\frac{a_{n}^{k}\left|d_{n}\right|}{(2 \pi)^{k}}\right]^{1 / 2}\left[\int_{|t| \geq \delta} \exp \left(a_{n}\left[f_{n}\left(\tau_{n}+i t\right)-f_{n}\left(\tau_{n}\right)\right]\right) \frac{\psi_{2 n}^{\prime}\left(-<r_{n}, \tau_{n}+i t>\right)}{\psi_{2 n}^{\prime}\left(-<r_{n}, \tau_{n}>\right)} d t\right.} \\
& \left.+\int_{|t|<\delta} \exp \left(a_{n}\left[f_{n}\left(\tau_{n}+i t\right)-f_{n}\left(\tau_{n}\right)\right]\right) \frac{\psi_{2 n}^{\prime}\left(-<r_{n}, \tau_{n}+i t>\right)}{\psi_{2 n}\left(-<r_{n}, \tau_{n}>\right)} d t\right]
\end{aligned}
$$

where $\delta$ is chosen such that $\delta=\min \left(\delta_{1}, \delta_{2}, \delta_{3}\right)$ and $M(\delta)<\alpha_{1} / 2 k$ for $M(\delta)$ in (3.45). Thus

$$
I_{n}=I_{n 1}+I_{n 2}
$$

where

$$
I_{n 1}=\left[\frac{a_{n}^{k}\left|d_{n}\right|}{2 \pi^{k}}\right]^{1 / 2} \int_{|t| \geq \delta} \exp \left(a_{n}\left[f_{n}\left(\tau_{n}+i t\right)-f_{n}\left(\tau_{n}\right)\right]\right) \frac{\psi_{2 n}^{\prime}\left(-<r_{n}, \tau_{n}+i t>\right)}{\psi_{2 n}\left(-<r_{n}, \tau_{n}>\right)} d t
$$


and

$I_{n 2}=\left[\frac{a_{n}^{k}\left|d_{n}\right|}{2 \pi^{k}}\right]^{1 / 2} \int_{|t|<\delta} \exp \left(a_{n}\left[f_{n}\left(\tau_{n}+i t\right)-f_{n}\left(\tau_{n}\right)\right]\right) \frac{\psi_{2 n}^{\prime}\left(-<r_{n}, \tau_{n}+i t>\right)}{\psi_{2 n}\left(-<r_{n}, \tau_{n}>\right)} d t$

Lemma 3.2.7 shows that the term $I_{n 1}$ goes exponentially fast to zero and Lemma 3.2.9 shows that $I_{n 2}=1+O\left(1 / a_{n}\right)$. Thus

$$
I_{n}=1+O\left(1 / a_{n}\right)
$$

This completes the proof of the theorem.

Theorem 3.2.10 stated below is analogous to Theorem 3.2.1 except that in Theorem 3.2.10 we take the components of $T_{n}$ and $S_{n}$ to be sums of $n$ i.i.d. random variables, particular case of Theorem 3.2.1.

Theorem 3.2.10 Let $\left\{X_{n}, n \geq 1\right\}$ be a sequence of non-lattice i.i.d. random vectors in $R^{k}$ with distribution function $F_{1}$ and m.g.f. $\phi_{1}$. Let $\left\{Y_{n}, n \geq 1\right\}$ be another sequence of non- lattice, positive random variables with distribution function $F_{2}$ and m.g.f. $\phi_{2}$. We assume that $Y_{n}^{\prime}$ s are independent of the components of $X_{n}^{\prime}$ s. Let $\phi_{1}$ and $\phi_{2}$ be analytic and non-vanishing in $\Omega_{1}^{k}$ and $\Omega_{2}$ respectively, where $\Omega_{i}=\left\{z \in C:|z|<c_{i}\right\}$ for $c_{i}>0, i=1,2$. Let

$$
\psi_{i}(z)=\frac{1}{n} \log \phi_{i}(z),
$$

for $i=1,2$. Let $J_{1}=\left(-b_{1}, b_{1}\right), 0<b_{1}<c_{1}$ and $J_{2}=\left(-b_{2}, b_{2}\right), 0<b_{2}<c_{2}$. Let $\left\{a_{n}\right\}$ be a sequence of real numbers such that $a_{n} \rightarrow \infty$.

Let $\nabla \psi_{1}(z)=\left(D_{1} \psi_{1}(z), D_{2} \psi_{1}(z), \cdots, D_{k} \psi_{1}(z)\right)$ be the vector of the first order partial derivatives and $\nabla^{2} \psi_{1}(z)$ denote the matrix of second order partial derivatives, that is,

$$
\nabla^{2} \psi_{1}(z)=\left(D_{i j} \psi_{1}(z)\right)
$$


The determinant of the matrix $\nabla^{2} \psi_{1}(z)$ is denoted by $\left|\nabla^{2} \psi_{1}(z)\right|$.

Let $d_{n}=\nabla^{2}\left(\psi_{1}(\tau)+\psi_{2}(-<r, \tau>)\right)$ be positive definite. Further, let the eigen values of $d_{n}$ be bounded below by $\alpha_{1}>0$, for all $\tau \in J_{1}^{k}$ and $n \geq 1$. Let $\left\{r_{n}\right\}$ be a sequence of vectors in $R^{k}$ such that $\left|r_{j n}\right|<r_{j}^{*}$ for $j=1, \cdots, k$ and $n \geq 1$. For this sequence $r_{n}$ let there exist a sequence $\left\{\tau_{n}\right\}$ in $J_{1}^{k}$ such that $<r_{n}, \tau_{n}>\in J_{2}$

$$
\nabla\left(\psi_{1}\left(\tau_{n}\right)+\psi_{2}\left(-<r_{n}, \tau_{n}>\right)\right)=0
$$

and

$$
\psi_{2}^{t}\left(-<r_{n}, \tau_{n}>\right)>\alpha_{2}
$$

where $\alpha_{2}>0$ and $n \geq 1$

Let us assume the following conditions on the moment generating functions $\phi_{1}$ and $\phi_{2}$.

(F11) There exist $\beta_{1}<\infty$ and $\beta_{2}<\infty$ such that

$$
\left|\psi_{1}(z)\right|<\beta_{1} \text { for } z \in \Omega_{1}^{k}
$$

and

$$
\left|\psi_{2}(z)\right|<\beta_{2} \text { for } z \in \Omega_{2} \text {. }
$$

(F12) Given $\delta>0$, there exists $q>0$ such that

$$
\sup _{|t|>\delta}\left|\psi_{2}^{\prime}\left(-<r_{n}, \tau_{n}+i t>\right)\right|=O\left(n^{q}\right)
$$

(F18) There exists $B_{1}<\infty$ such that

$$
\sup _{\tau \in J_{1}^{k}} \int_{-\infty}^{\infty}\left|\frac{\phi_{1}(\tau+i t)}{\phi_{1}(\tau)}\right|^{l / a_{n}} d t<B_{1} \text {. }
$$


(F12) Given $\delta>0$, there exists $q>0$ such that

$$
\sup _{|t|>\delta}\left|\psi_{2}^{\prime}\left(-<r_{n}, \tau_{n}+i t>\right)\right|=O\left(n^{q}\right)
$$

(F18) There exists $B_{1}<\infty$ such that

$$
\sup _{\tau \in J_{1}^{k}} \int_{-\infty}^{\infty}\left|\frac{\phi_{1}(\tau+i t)}{\phi_{1}(\tau)}\right|^{l / a_{n}} d t<B_{1} \text {. }
$$

Let $T_{n}=X_{1}+\cdots+X_{n}, S_{n}=Y_{1}+\cdots+Y_{n}$. Then an asymptotic expression for the p.d.f. of $R_{n}=T_{n} / S_{n}$ at the point $r_{n}$ is given by,

$$
\begin{aligned}
\bar{G}_{1}\left(r_{n}\right)= & \frac{n^{k / 2}\left(\psi_{2}^{\prime}\left(-<r_{n}, \tau_{n}>\right)\right)^{k}}{(2 \pi)^{k / 2}\left|d_{n}\right|^{1 / 2}} \\
& \times \exp \left(n\left[\psi_{1}\left(\tau_{n}\right)+\psi_{2}\left(-<r_{n}, \tau_{n}>\right)\right]\right)[1+O(1 / n)] .
\end{aligned}
$$




\subsection{Large deviation local limit theorems for lattice random vectors}

Now we proceed to obtain similar local limit theorems for ratio statistic $R_{n}=T_{n} / S_{n}$ where the random vector $T_{n}$ and the random variable $S_{n}$ both are lattice. We continue to use the same notations introduced in Section 3.2 .

Before we state and prove the results of this section we give below a few definitions and results for lattice random vectors.

Definition 3.3.1. Consider $R^{k}$ as a group under vector addition. A subgroup $L$ is discrete if every ball in $R^{k}$ has only a finite number of points of $L$ in it.

Definition 3.3.2. A discrete subgroup $L$ is called a lattice if there exists $k$ linearly independent vectors $\left\{\xi_{1}, \ldots, \xi_{\dot{k}}\right\}$ such that

$L=\left[\xi: \xi=m_{1} \xi_{1}+\cdots+m_{k} \xi_{k} ; m_{i}\right.$ integer, $\left.\forall i=1, \ldots, k\right]$.

Definition 3.3.3. A random vector $X$ in $R^{k}$ is a lattice random vector if there exists $x_{0} \in R^{k}$ and a lattice such that $\operatorname{Pr}\left(X \in x_{0}+L\right)=1$.

Definition 3.3.4. A random vector $X$ in $R^{k}$ is degenerate if there exists a hyperplane $H=\{x:\langle a, x\rangle=c\}$, where $a \neq 0$ and $c$ is a constant such that $\operatorname{Pr}(X \in H)=1$, otherwise we say that $X$ is non-degenerate.

Lemma 3.3.5. Let $X$ be a nondegenerate lattice random vector. Then there exists a unique lattice $L_{0}$ such that the following two properties hold: 
(i) $\operatorname{Pr}\left(X \in x+L_{0}\right)=1$ for all $x$ such that $\operatorname{Pr}(X=x)>0$.

(ii) If $M$ is any closed subgrouph such that $\operatorname{Pr}\left(X \in y_{0}+M\right)=1$ for some $y_{0} \in R^{k}$, then $L_{0} \subset M$.

Proof: See Bhattacharya and Ranga Rao (1976), pp. 226-227.

The lattice $L_{0}$ is called the minimal lattice for $X$. In what follows we shall consider only the minimal lattice. Let $\phi(t)=E e^{i<t, x>}, t \in R^{k}$ be the characteristic function of $X$. A vector $t_{0} \in R^{k}$ is said to be a period of $|\phi|$ if $\left|\phi\left(t+t_{0}\right)\right|=|\phi(t)|$ for all $t \in R^{k}$.

Lemma 3.3.6. Let $X$ be a lattice random vector with characteristic function $\phi$. The set $L_{1}$ of periods of $|\phi|$ is a lattice if and only if $X$ is nondegenrate. Further, let $L_{1}=\{t:\langle t, \xi\rangle \in 2 \pi m \forall \xi \in L, m$ integer $\}$. Let $\left\{n_{1}, \ldots, n_{k}\right\}$ be a dual basis, that is, $\left\langle\xi_{j}, n_{j^{\prime}}\right\rangle=0$ if $j \neq j^{\prime}$ and 1 if $j=j^{\prime}$. Then $L_{1}=\left\{2 \pi t: t=m_{1} n_{1}+\cdots+m_{k} n_{k}, m_{i}\right.$ integer $\}$.

Proof. See Bhattacharya and Ranga Rao (1976), pp. 227-228.

This completes the preliminaries. We now proceed with Theorem 3.3.7 which gives an asymptotic expression for the density of the ratio statistic $R_{n}=T_{n} / S_{n}$ where $\left\{T_{n}, n \geq 1\right\}$ is a sequence of lattice random vectors and $\left\{S_{n}, n \geq 1\right\}$ is a sequence of lattice valued random variables.

Theorem 3.3.7. Let $L_{n}$ be the lattice $\left\{\xi: \xi=h_{r}\left(m_{1} e_{1}+\ldots+m_{k} e_{k}\right), m_{:}\right.$integer $\}$, where $\left\{h_{n}, n \geq 1\right\}$ is a sequence of real numbers and $\left\{e_{1}, \cdots, e_{k}\right\}$ is an orthonormal basis of $R^{k}$. Let $\left\{T_{n}, n \geq 1\right\}$ be a sequence of nondegenrate 
lattice random vectors defined on $L_{n}$ with distribution functions $F_{1 n}$. Let $\left\{S_{n}, n \geq 1\right\}$ be a sequence of lattice random variables taking values in the set $S^{\prime}=\left\{a_{n}^{\prime}+k h_{n}^{\prime}: a_{n}^{\prime} h_{n}^{\prime}\right.$ are such that $\left.a_{n}^{\prime}+k h_{n}^{\prime}>0\right\}$. Let $S_{n}$ be independent of the components of the vector $T_{n}$ for all $n \geq 1$. Let $r_{n}=\left(r_{1 n}, \cdots, r_{k n}\right)$ be a sequence of vectors in $R^{k}$ such that $\left|r_{j n}\right|<r$ for $j=1, \cdots, k$ and $n \geq 1$ and $r>0$.

Assume that the following conditions are satisfied.

(H1) There exist $\beta_{1}<\infty$ and $\beta_{2}<\infty$ such that

$$
\left|\psi_{1 n}(z)\right|<\beta_{1} \text { for } z \in \Omega_{1}^{k}
$$

and

$$
\left|\psi_{2 n}(z)\right|<\beta_{2} \text { for } z \in \Omega_{2}
$$

for $n \geq 1$.

(H2) Given $\delta>0$, there exists $0<\eta<1$ such that

$$
\limsup \sup _{\delta \leq|t| \leq \pi /\left|h_{n}\right|}\left|\frac{\phi_{1 n}\left(\tau_{n}+i t\right)}{\phi_{1 n}\left(\tau_{n}\right)}\right|^{1 / a_{n}}<\eta .
$$

(H3) There exist $p, l>0$ such that

$$
\sup _{\tau \in J_{1}^{k}} \int_{-\pi /\left|h_{n}\right|}^{\pi /\left|h_{n}\right|}\left|\frac{\phi_{1 n}\left(\tau_{n}+i t\right)}{\phi_{1 n}\left(\tau_{n}\right)}\right|^{1 / a_{n}}=O\left(a_{n}^{p}\right) .
$$

Let $P_{n}\left(r_{n}\right)=P\left(T_{n}=r_{n} S_{n}\right)$. Then

$$
\begin{aligned}
P_{n}\left(r_{n}\right)= & {\left[\frac{\left|h_{n}\right|^{k}}{(2 \pi)^{k} a_{n}^{k}\left|\nabla^{2}\left(\psi_{1 n}\left(\tau_{n}\right)+\psi_{2 n}\left(-<r_{n}, \tau_{n}>\right)\right)\right|}\right]^{1 / 2} } \\
& \times \exp \left(a_{n}\left[\psi_{1 n}\left(\tau_{n}\right)+\psi_{2 n}\left(-<r_{n}, \tau_{n}>\right)\right]\right)\left[1+O\left(1 / a_{n}\right)\right] .
\end{aligned}
$$


Proof: Consider,

$$
P\left(T_{n}=r_{n} S_{n}\right)=\sum_{y \in S^{\prime}, r y \in L_{n}} P\left(T_{n}=r y\right) P\left(S_{n}=y\right) .
$$

We use the conjugate distribution technique to obtain the probability $P\left(T_{n}=\right.$ $r y$ ) and it is given by

$$
P\left(T_{n}=r y\right)=\frac{\left|h_{n}\right|^{k}}{(2 \pi)^{k}} \int_{E^{n}} \phi_{1 n}(\tau+i t) e^{-<r y, \tau+i t\rangle} d t
$$

where $E^{n}=\left\{\left(t_{1}, \cdots, t_{k}\right):\left|t_{j}\right|<\pi /\left|h_{n}\right|\right\}$. In view of (3.82), (3.81) becomes

$$
\begin{aligned}
P\left(T_{n}=r S_{n}\right) & =\sum_{y \in S^{\prime}, r y \in L_{n}}\left[\frac{\left|h_{n}\right|^{k}}{(2 \pi)^{k}} \int_{E^{n}} \phi_{1 n}(\tau+i t) e^{-<r y, \tau+i t>} d t\right] P\left(S_{n}=y\right) \\
& =\left[\frac{\left|h_{n}\right|^{k}}{(2 \pi)^{k}}\right] \int_{E^{n}} \phi_{1 n}(\tau+i t) \sum_{y \in S^{\prime}, r y \in L_{n}} e^{-<r y, \tau+i t>} P\left(S_{n}=y\right) \\
& =\left[\frac{\left|h_{n}\right|^{k}}{(2 \pi)^{k}}\right] \int_{E^{n}} \phi_{1 n}(\tau+i t) \phi_{2 n}(-<r y, \tau+i t>) d t \\
& =\left[\frac{\left|h_{n}\right|^{k}}{(2 \pi)^{k}}\right] \int_{E^{n}} \exp \left(a_{n}\left[\psi_{1 n}(\tau+i t)+\psi_{2 n}(-<r y, \tau+i t>)\right] d t\right.
\end{aligned}
$$

The saddle point allows us to replace $\tau$ by $\tau_{n}$ when $r$ is replaced by $r_{n}$. Thus, we have,

$$
\begin{aligned}
P\left(T_{n}=r_{n} S_{n}\right) & =\left[\frac{\left|h_{n}\right|^{k}}{(2 \pi)^{k}}\right] \int_{E^{n}} \exp \left(a_{n}\left[\psi_{1 n}\left(\tau_{n}+i t\right)+\psi_{2 n}\left(-<r_{n} y, \tau_{n}+i t>\right)\right] d t\right. \\
& =\left[\frac{\left|h_{n}\right|^{k}}{a_{n}^{k / 2}(2 \pi)^{k / 2}\left|d_{n}\right|^{1 / 2}}\right] \int_{E^{n}} \exp \left(a_{n}\left[\psi_{1 n}\left(\tau_{n}\right)+\psi_{2 n}\left(-<r_{n}, \tau_{n}>\right)\right]\right) I_{n}
\end{aligned}
$$

where

$I_{n}=\left[\frac{a_{n}^{k / 2}\left|d_{n}\right|^{1 / 2}}{2 \pi^{k / 2}}\right] \int_{E^{n}} \exp \left(a_{n}\left[\psi_{1 n}\left(\tau_{n}+i t\right)-\psi_{1 n}\left(\tau_{n}\right)+\psi_{2 n}\left(-<r_{n}, \tau_{n}+i t>\right)\right.\right.$ 


$$
\left.\left.-\psi_{2 n}\left(-<r_{n}, \tau_{n}>\right)\right]\right) d t
$$

Using the techniques similar to the techniques in Theorem 3.3.7, we can easily show that $I_{n}=1+O\left(1 / a_{n}\right)$. Thus

$$
\begin{aligned}
P\left(T_{n}=\tau_{n} S_{n}\right)= & {\left[\frac{\left|h_{n}\right|^{k}}{a_{n}^{k / 2}(2 \pi)^{k / 2}\left|d_{n}\right|^{1 / 2}}\right] } \\
& \times \exp \left(a_{n}\left[\psi_{1 n}\left(\tau_{n}\right)+\psi_{2 n}\left(-<\tau_{n}, \tau_{n}>\right)\right]\right)\left[1+O\left(1 / a_{n}\right)\right] .
\end{aligned}
$$

The following Theorem 3.3.8 is a particular case of Theorem 3.3.7 proved above. Here $T_{n}$ is the sum of $n$ i.i.d. lattice valued random vectors defined on the lattice $L_{n}$ and $S_{n}$ is the sum of $n$ i.i.d. lattice valued random variables $Y_{n}$. This theorem gives an asymptotic expression for the density of the ratio of two statistics.

Theorem 3.3.8. Let $L_{n}$ be the lattice $\left\{\xi: \xi=h_{n}\left(m_{1} e_{1}+\ldots+m_{k} e_{k}\right), m_{i}\right.$ integer $\}$, where $\left\{h_{n}, n \geq 1\right\}$ is a sequence of real numbers and $\left\{e_{1}, \cdots, e_{k}\right\}$ is an orthonormal basis of $R^{k}$. Let $\left\{X_{n}, n \geq 1\right\}$ be a sequence of i.i.d. lattice valu ed random vectors defined on lattice $L_{n}$. Let $\left\{Y_{n}, n \geq 1\right\}$ be an i.i.d. sequence of lattice random variables taking values in the set $S^{\prime}=\left\{a_{n}^{\prime}+k h_{n}^{\prime}\right.$ : $a_{n}^{\prime} h_{n}^{\prime}$ are such that $\left.a_{n}^{\prime}+k h_{n}^{\prime}>0\right\}$. Let $Y_{n}$ be independent of the components of the random vectors $X_{n}$ for all $n \geq 1$. Let $r_{n}=\left(r_{1 n}, \cdots, r_{k n}\right)$ be $a$ sequence of vectors in $R^{k}$ such that $\left|r_{j n}\right|<r$ for $j=1, \cdots, k$ and $n \geq 1$ and $r>0$. For this sequence $\left\{r_{n}\right\}$, let there exist $\tau_{n}^{\prime} s$ in $J_{1}^{k}$ such that

$$
\psi_{1}^{\prime}\left(\tau_{n}\right)-r_{n} \psi_{2}^{\prime}\left(-<r_{n}, \tau_{n}>\right)=0 \text { and }<r_{n}, \tau_{n}>\in J_{2}
$$


Let $v_{n}=\nabla^{2}\left(\psi_{1}(\tau)+\psi_{2}(-<r, \tau>)\right)$ for $\tau \in J_{1}^{k}$ and $<r, \tau>\in J_{2}$. Let the eigen values of $v_{n}$ be bounded below by $\alpha_{1}>0$. Assume that the following conditions are satisfied.

(H11) There exist $\beta_{1}<\infty$ and $\beta_{2}<\infty$ such that

$$
\left|\psi_{1}(z)\right|<\beta_{1} \text { for } z \in \Omega_{1}^{k}
$$

and

$$
\left|\psi_{2}(z)\right|<\beta_{2} \text { for } z \in \Omega_{2}
$$

(H19) There exists $B_{3}$ such that

$$
\sup _{\tau \in J_{1}^{k}} \int_{-\pi /|h|}^{\pi /|h|}\left|\frac{\phi_{1}(\tau+i t)}{\phi_{1}(\tau)}\right|^{n}<B_{3}
$$

Let $T_{n}=X_{1}+\cdots+X_{n}$ and $S_{n}=Y_{1}+\cdots+Y_{n}$. Let $P\left(T_{n}=r_{n} S_{n}\right)=P_{n}\left(r_{n}\right)$. Then,

$$
\begin{aligned}
P_{n}\left(r_{n}\right)= & {\left[\frac{|h|^{k}}{(2 \pi)^{k} n^{k}\left|\nabla^{2}\left(\psi_{1}(\tau)+\psi_{2}(-<r, \tau>)\right)\right|}\right]^{1 / 2} } \\
& \times \exp \left(a\left[\psi_{1}(\tau)+\psi_{2}(-<r, \tau>)\right]\right)[1+O(1 / n)]
\end{aligned}
$$




\section{Chapter 4}

\section{Strong large deviation results}

\section{for ratio statistics}

\subsection{Introduction}

Let $\left\{T_{n}, n \geq 1\right\}$ be a sequence of random variables such that $T_{n} / a_{n} \rightarrow 0$ for some sequence of real numbers $a_{n} \rightarrow \infty$. In most examples the probability of the event $\left\{T_{n} \geq x_{n}\right\}$ goes to zero exponentially fast whenever $x_{n}=O\left(a_{n}\right)$ and $x_{n}$ is positive. The event $\left\{T_{n} \geq x_{n}\right\}$ for $x_{n}$ positive is known in the literature as a large deviation event. Numerous authors including Cramer (1938), Chernoff (1952), Ellis (1984), Varadhan (1984) have obtained asymptotic expressions for $\log P\left(T_{n} \geq x_{n}\right)$ under some conditions on the moment generating function of $T_{n}$. Bahadhur and Ranga Rao (1960) obtained asymptotic expression for $P\left(T_{n} \geq x_{n}\right)$ when $T_{n}$ is the sum of $n$ i.i.d. random variables and $x_{n}=O(n)$. Chaganty- Sethuraman coined the 
term Weak large deviation results for the results of the former type and Strong large deviation results for the results of the latter type. In a recent paper, Chaganty-Sethuraman extended the results of Bahadhur and Ranga Rao (1960) to an arbitrary sequence of random variables $T_{n}$, not necessarily sums of i.i.d. random variables. We state these results precisely in Theorems 4.2.2 and 4.3.2.

The purpose of this chapter is to obtain strong large deviation result for the ratio statistic $R_{n}=T_{n} / S_{n}$, where $T_{n}$ and $S_{n}$ are arbitrary sequences of random variables. In Theorem 4.2.3, we show that Conditions $(Q 1)$ and (Q2) on the m.g.f.'s of $T_{n}$ and $S_{n}$ imply Conditions (P1) and (P2) of theorem 4.2.2 for the random variable $T_{n}^{\prime}$. Thus Conditions (P1) and (P2) of Theorem 4.2.2 are satisfiesd for arbitrary random variables $T_{n}^{\prime}=T_{n}-r_{n} S_{n}$ giving an asymptotic expression for $P\left(T_{n} / S_{n} \geq r_{n}\right)$ i.e. for $P\left(T_{n}-r_{n} S_{n} \geq 0\right)$. The case of arbitrary lattice random variables $T_{n}$ and $S_{n}$ is treated in Theorem 4.3.3.

\subsection{Non-lattice case}

This section contains strong large deviation limit theorems for non-lattice random variables.

Strong large deviation theorem for an arbitrary sequence of non- lattice random variables is stated below in Theorem 4.2.2.

Theorem 4.2.1 Let $\left\{W_{n}, n \geq 1\right\}$ be a sequence of nonlattice valued random variables with m.g.f. $\phi_{n}(z)$. Let $\phi_{n}(z)$ be analytic and nonvanishing in 
$\Omega=\{z \in C:|z|<c\}$, where $c>0$. Let

$$
\psi_{n}(z)=\frac{1}{a_{n}} \phi_{n}(z)
$$

for $z \in \Omega, n \geq 1$. Also, let

$$
\gamma_{n}(u)=\sup _{|s|<a}\left[u s-\psi_{n}(s)\right], \text { for real } u .
$$

Let $\left\{m_{n}, n \geq 1\right\}$ be a sequence of real numbers such that there exists a sequence $\tau_{n}$ satisfying $\psi_{n}^{\prime}\left(\tau_{n}\right)=m_{n}$ and $d<\tau_{n}<c<c_{1}$ for some positive numbers $c$ and $d$ and for all $n \geq 1$. Assume the following conditions for $T_{n}$

(P1) There exists $\beta<\infty$ such that $\left|\psi_{n}(z)\right|<\beta$ for all $n \geq 1, z \in \Omega$.

(P2) There exists $\delta_{1}>0$ such that

$$
\sup _{|t| \geq \delta}\left|\frac{\phi_{n}\left(\tau_{n}+i t\right)}{\phi_{n}\left(\tau_{n}\right)}\right|=o\left(\frac{1}{\sqrt{a_{n}}}\right)
$$

for all $0<\delta<\delta_{1}$.

(PS) There exists $\alpha>0$ such that $\psi_{n}^{\prime \prime}\left(\tau_{n}\right) \geq \alpha$ for all $n \geq 1$.

Then

$$
P\left(\frac{W_{n}}{a_{n}} \geq m_{n}\right) \sim \frac{\exp \left(-a_{n} \gamma_{n}\left(m_{n}\right)\right)}{\sqrt{2 \pi} \tau_{n} \sqrt{a_{n} \psi_{n}^{\prime \prime}\left(\tau_{n}\right)}} .
$$

The following Theorem 4.2.3 generalizes Theorem 4.2.2 stated above to ratio statistic.

Theorem 4.2.2 Let $\left\{T_{n}, n \geq 1\right\}$ be an arbitrary sequence of nonlattice random variables with m.g.f. $\phi_{1 n}(z)=E\left[\exp \left(z T_{n}\right)\right]$ and $\left\{S_{n}, n \geq 1\right\}$ be another sequence of noniattice positive random variables with m.g.f. $\phi_{2 n}(z)=$ 
$E\left[\exp \left(z T_{n}\right)\right]$. Assume that the two sequences are independent of each other. Let $\phi_{1 n}$ and $\phi_{2 n}$ be nonvanishing and analytic in the region $\Omega=\{z \in C$ : $|z|<c\}$, where $c>0$ and $C$ is the set of all complex numbers. Let $\left\{a_{n}\right\}$ be a sequence of real numbers such that $a_{n} \rightarrow \infty$. Let

$$
\psi_{i n}(z)=\frac{1}{a_{n}} \log \phi_{i n}(z), \text { for } z \in \Omega, i=1,2 .
$$

Let $\left\{r_{n}\right\}$ be a positive bounded sequence of real numbers such that there exists $\tau_{n}$ satisfying $0<d<\tau_{n}<c$ and

$$
\psi_{1 n}^{\prime}\left(\tau_{n}\right)-r_{n} \psi_{2 n}^{\prime}\left(-r_{n} \tau_{n}\right)=0
$$

Assume the following conditions for $T_{n}$ and $S_{n}$ :

(Q1) There exist $\beta_{i}<\infty$ such that $\left|\psi_{i}(z)\right|<\beta_{i}$ for $z \in \Omega$, for $i=1,2$.

(Q2) There exists $\delta_{1}>0$ such that

for any $0<\delta<\delta_{1}$.

$$
\sup _{|t| \geq \delta}\left|\frac{\phi_{1 n}\left(\tau_{n}+i t\right)}{\phi_{1 n}\left(\tau_{n}\right)}\right|=o\left(1 / a_{n}\right)
$$

(QS) Let $\alpha_{1}$ be a positive real number such that $\psi_{1 n}^{\prime \prime}(\tau)>\alpha_{1}$.

Then

$$
P\left(\frac{T_{n}}{S_{n}} \geq r_{n}\right) \sim \frac{\exp \left(a_{n}\left[\psi_{1 n}\left(\tau_{n}\right)+\psi_{2 n}\left(-r_{n} \tau_{n}\right)\right]\right)}{\sqrt{2 \pi} \tau_{n} \sqrt{a_{n}\left[\psi_{1 n}^{\prime \prime}\left(\tau_{n}\right)+r_{n}^{2} \psi_{2 n}^{\prime \prime}\left(-\tau_{n} \tau_{n}\right)\right]}}
$$

Proof: Consider

$$
\begin{aligned}
P\left(\frac{T_{n}}{S_{n}} \geq r_{n}\right) & =P\left(T_{n}-r_{n} S_{n} \geq 0\right) \\
& =P\left(\frac{T_{n}^{*}}{a_{n}} \geq 0\right)
\end{aligned}
$$


where $T_{n}^{*}=T_{n}-r_{n} S_{n}$. Using the independence of $T_{n}$ and $S_{n}$ we get the follwing relationship between the m.g.f.'s of $T_{n}, S_{n}$ and $T_{n}^{*}$

$$
\phi_{0 n}(\tau)=\phi_{1 n}(\tau) \phi_{2 n}(-r \tau) \text { for } \tau \in J_{0}
$$

which implies that

$$
\psi_{0 n}(z)=\psi_{1 n}(z)+\psi_{2 n}(-r z)
$$

where $\phi_{0 n}$ is the m.g.f. of $T_{n}^{*}, \psi_{0 n}(z)=\frac{1}{a_{n}} \log \phi_{0 n}(z)$. Let $\gamma_{n}^{*}(u)=\sup _{|s|<c}[u s-$ $\left.\psi_{0 n}(s)\right]$. From Condition $(Q 1)$ and (4.11), it follows that

$$
\left|\psi_{0 n}(z)\right|<\beta_{1}+\beta_{2}
$$

Thus, there exists $\beta_{0}=\beta_{1}+\beta_{2}<\infty$ such that $\left|\psi_{0 n}(z)\right|<\beta_{0}$. Hence Condition (P1) of Theorem 4.2.2 is satisfied.

We have for any $0<\delta<\delta_{1}$

$$
\begin{aligned}
\sup _{|t| \geq \delta}\left|\frac{\phi_{0 n}\left(\tau_{n}+i t\right)}{\phi_{0 n}\left(\tau_{n}\right)}\right| & =\sup _{|t| \geq \delta}\left|\frac{\phi_{1 n}\left(\tau_{n}+i t\right)}{\phi_{1 n}\left(\tau_{n}\right)} \frac{\phi_{2 n}\left(\tau_{n}+i t\right)}{\phi_{2 n}\left(\tau_{n}\right)}\right| \\
& \leq \sup _{|t| \geq \delta}\left|\frac{\phi_{1 n}\left(\tau_{n}+i t\right)}{\phi_{0 n}\left(\tau_{n}\right)}\right| .
\end{aligned}
$$

From Condition $(Q 2)$ it follows that

$$
\sup _{|t| \geq \delta}\left|\frac{\phi_{0 n}\left(\tau_{n}+i t\right)}{\phi_{0_{n}}\left(\tau_{n}\right)}\right|=o\left(1 / a_{n}\right) \text {. }
$$

Hence Condition (P2) of Theorem 4.2.2 is also satisfied. In view of $\psi_{1 n}^{\prime \prime}(\tau)>$ $\alpha_{1}$ and (4.11) Condition (P3) is easily satisfied. Thus an arbitrary sequence $\left\{T_{n}^{*}, n \geq 1\right\}$ satisfies all the conditions of Theorem 4.2.2 and hence the conclusion of the Theorem 4.2.2 yields

$$
P\left(\frac{T_{n}^{*}}{a_{n}} \geq 0\right) \sim \frac{\exp \left(-a_{n} \gamma_{n}^{*}(0)\right)}{\sqrt{2 \pi} \tau_{n} \sqrt{a_{n} \psi_{0 n}^{\prime \prime}\left(\tau_{n}\right)}}
$$


which in turn gives Strong large deviation result for $T_{n} / S_{n}$, namely,

$$
P\left(\frac{T_{n}}{S_{n}} \geq r_{n}\right) \sim \frac{\exp \left(a_{n}\left[\psi_{1 n}\left(\tau_{n}\right)+\psi_{2 n}\left(-r_{n} \tau_{n}\right)\right]\right)}{\sqrt{2 \pi} \tau_{n} \sqrt{a_{n}\left[\psi_{1 n}^{\prime \prime}\left(\tau_{n}\right)+r_{n}^{2} \psi_{2 n}^{\prime \prime}\left(-r_{n} \tau_{n}\right)\right]}} .
$$

\subsection{The Lattice case}

In this section we state strong large deviation theorem for lattice valued random variables. These theorems are analogous to the theorems for lattice valued random variables of the previous section.

The next theorem provides an estimate of $P\left(T_{n} / a_{n} \geq m_{n}\right)$ where $\left\{T_{n}, n \geq 1\right\}$ is a sequence of lattice valued random variables and $m_{n}$ is in the large deviation of $T_{n} / a_{n}$. We first introduce few notations.

Let $\left\{T_{n}, n \geq 1\right\}$ be an arbitrary sequence of lattice valued random variables taking values in the lattice $\left\{t_{n}+k p_{n}: k=0, \pm 1, \pm 2, \cdots\right\}, p_{n}>0$. Let the c.f. of $T_{n}, \phi_{n}(z)$, be analytic and nonvanishing in the region $\Omega=\left\{z \in C:|z|<c_{1}\right\}$. Let $\left\{a_{n}\right\}$ be a sequence of real numbers such that $a_{n} \rightarrow \infty$ and $p_{n}=o\left(\sqrt{a_{n}}\right)$. Let

$$
\psi_{n}(z)=\frac{1}{a_{n}} \log \phi_{n}(z)
$$

be a well defined analytic function on $\Omega$. Let $\left\{m_{n}\right\}$ be a sequence of real numbers such that there exists $0<\tau_{n}<b_{1}<c_{1}$ satisfying $\psi_{n}^{\prime}\left(\tau_{n}\right)=m_{n}$ and $\tau_{n} \sqrt{a_{n}} \rightarrow \infty$ as $n \rightarrow \infty$. We now state Theorem 4.3.2.

Theorem 4.3.1 Assume that $T_{n}$ satisfies Conditions (P1) and (PS) of Theorem 4.2.2 and the following Condition (P2'): 
(P2') There exists $\delta_{1}>0$, such that for $0<\delta<\delta_{1}$,

$$
\sup _{\delta \leq|t|<\pi / h_{n}}\left|\frac{\phi_{n}\left(\tau_{n}+i t\right)}{\phi_{n}\left(\tau_{n}\right)}\right| .
$$

Then

$$
P\left(\frac{T_{n}}{a_{n}} \geq m_{n}\right) \sim \frac{p_{n}}{\sqrt{2 \pi} \sqrt{a_{n} \psi_{n}^{\prime \prime}\left(\tau_{n}\right)}} \frac{\exp \left(-a_{n} \gamma_{n}\left(m_{n}\right)\right)}{\left(1-\exp \left(-p_{n} \tau_{n}\right)\right)}
$$

where $\gamma_{n}\left(m_{n}\right)=m_{n} \tau_{n}-\psi_{n}\left(\tau_{n}\right)$.

Next, we prersent Theorem 4.3.3 which provides an estimate of the large deviation probability for the ratio $T_{n} / S_{n}$, where $\left\{T_{n}, n \geq 1\right\}$ and $\left\{S_{n}>0, n \geq 1\right\}$ denote the arbitrary sequences of random variables. First we begin with few preliminaries.

Let $\left\{T_{n}, n \geq 1\right\}$ be a sequence of lattice random variables with distribution functions $F_{1 n}$. Let $T_{n}$ take values in the set

$S_{1}=\left\{a_{n}+k h_{n}: a_{n}\right.$ and $h_{n}$ are real numbers and $k$ is an integer $\}$. Let $\left\{S_{n}>0, n \geq 1\right\}$ be a sequence of positive lattice random variables taking values in the set $S_{2}=\left\{a_{n}^{\prime}+k h_{n}^{\prime}: a_{n}^{\prime}\right.$ and $h_{n}^{\prime}$ are such that $\left.a_{n}^{\prime}+k h_{n}^{\prime}>0\right\}$. Let the m.g.f.'s of $T_{n}$ and $S_{n}, \phi_{1 n}$ and $\phi_{2 n}$, be analytic and non-vanishing in $\Omega=\{z \in C:|z|<b\}$. Let $r_{n}$ be a positive bounded sequence of real numbers such that there exist $\tau_{n}$ such that $0<\tau_{n}<b_{1}<b$ and

$$
\psi_{1 n}^{\prime}\left(\tau_{n}\right)-\tau_{n} \psi_{2 n}^{\prime}\left(-r_{n} \tau_{n}\right)=0
$$

for all $n \geq 1$.

Theorem 4.3.2 Assume that these two sequences meet the following conditions: (R1) There exist $\beta_{1}<\infty$ and $\beta_{2}<\infty$ such that

$$
\left|\psi_{j n}(z)\right|<\beta_{j} \text { for } z \text { with }|z|<b \text { for } j=1,2 \text { and } n \geq 1 \text {. }
$$


(R2) There exists $\delta_{1}>0$ such that

$$
\sup _{\delta \leq|t|<\pi / h_{n}}\left|\frac{\phi_{1 n}\left(\tau_{n}+i t\right)}{\phi_{1 n}\left(\tau_{n}\right)}\right|=o\left(1 / a_{n}\right)
$$

for any $0<\delta<\delta_{1}$.

(RS) Let $\alpha_{1}$ be positive real numbers such that

$$
\psi_{1 n}^{\prime \prime}(\tau)>\alpha_{1}
$$

Then

$$
\begin{aligned}
P\left(\frac{T_{n}}{S_{n}} \geq r_{n}\right) \sim & \frac{h_{n}}{\sqrt{2 \pi} \sqrt{a_{n}\left[\psi_{1 n}^{\prime \prime}\left(\tau_{n}\right)+r_{n}^{2} \psi_{2 n}^{\prime \prime}\left(-r_{n} \tau_{n}\right)\right]}} \\
& \times \frac{\exp \left(a_{n}\left[\psi_{1 n}\left(\tau_{n}\right)+\psi_{2 n}\left(-r_{n} \tau_{n}\right)\right]\right)}{\left(1-\exp \left(-h_{n} \tau_{n}\right)\right)} .
\end{aligned}
$$

Proof. The proof of this theorerm is similar to the proof of theorem and hence we omit it. 


\section{References}

[1] Apostol, T. M. (1974), Mathematical Analysis, Addison-Wesley.

[2] Bahadur, R. R. and Ranga, Rao (1960). On Deviations of the sample mean. Ann. Math. Statist., 31, 1015-1027.

[3] Berry, A. C. (1941). The accuracy of the Gaussian approximation to the sum of independent variables, Trans. Amer. Math. Soc., 49, 122-136.

[4] Chaganty, N. R. and Sethuraman, J. (1985). Large deviation local limit theorems for arbitrary sequences of random variables. Ann. of Probab., 13, 97-114.

[5] Chaganty, N. R. and Sethuraman, J. (1986). Multi-dimensional large deviation local limit theorems. Journal of Multi. Analysis, 20, 190-204.

[6] Chaganty, N. R. and Sethuraman, J. (1987). Strong Large and Local limit theorems, To appear in Ann. of Probab.

[7] Chernoff, H. (1952). A measure of asymptotic efficiency for tests of an hypothesis based on the sum of observations, Ann. Math. Statist., 23, 493-507.

[8] Conway, J. B. (1978). Functions of One Complex Variable, SpringerVerlag, New York.

[9] Chung, K. L. (1974). A course in Probability Theory. Academic Press, New York. 
[10] Cramer, H. (1938). Sur un nouveau theoreme - limit de la theorie des probabilites, Actual. Sci. et Ind., No. 736, Paris.

[11] Ellis, R. S. (1984). Large deviations for a general class of dependent random vectors, Ann. Probab. 12, 1-12.

[12] Esseen, C. G. (1945). Fourier analysis of distribution functions. A mathematical study of the Laplace Gaussian law, Acta. Math. 77, 1-125.

[13] Gnedenko, B. V. and Kolmogorov, A. N. (1954). Limit distributions for sums of independent random variables. Translated by K. L. Chung, Addison-Wesley.

[14] MacDonald. D. (1979). A local limit theorem for large deviations of sums of independent, nonidentically distributed random variables. Ann. of Probab. 7, 526-531.

[15] Moskvin. D. A. (1975). A local limit theorem for large deviations in the case of differently distributed lattice summands. Theor. Prob. Appl., $17,678-684$.

[16] Petrov, V. V. (1954). Uspekhi Matem. Nauk. Vol.9.

[17] Ranga, Rao and Varadarajan (1960). A limit theorem for densities, Sankhya, 22, 261-266.

[18] Richter, W. (1957). Local limit theorems for large deviations, Theor. Prob. Appl., 2, 206-219. 


\section{Autobiographical Statement}

Place and Date of Birth : KOLHAPUR (INDIA), August 28, 1958.

Educational :

Employment :
M.Sc., 1981, University of Poona, India.

B.Sc., 1979, Abasaheỉ Garware College, University of Poona, India.

Research Assistant, Dept. of Math.and Stat., Old

Dominion University. From: August, 1984 - May, 1985

and August, 1986 - May, 1987.

Teaching Assistant, Dept. of Math. and Stat., Old

Dominion University. From : August, 1983 - May, 1984

and August, 1985 - May,1986.

Statistician, Kirloskar Oil Engines Ltd., Poona,

India. From : July, 1981 - August, 1982. 